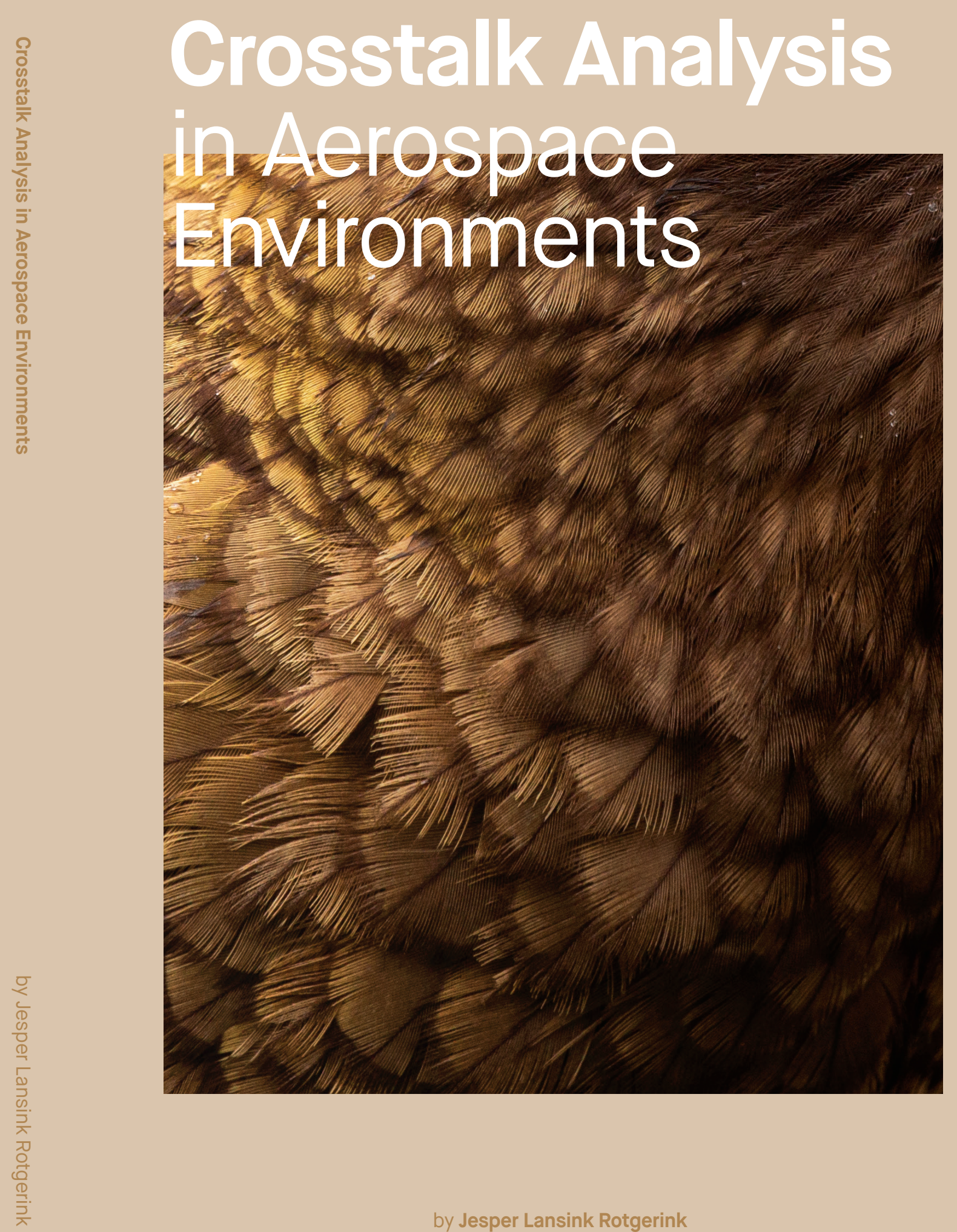




\section{CROSSTALK ANALYSIS IN AEROSPACE ENVIRONMENTS}

by

Jesper Lansink Rotgerink 


\section{Members of the graduation committee:}

\section{Chairman \& Secretary:}

Prof. dr. J. N. Kok

\section{Promoter:}

Prof. dr. ir. F. B. J. Leferink

\section{Internal members:}

Prof. dr. ir. G. Rietveld

Dr. A. Glazunov

\section{External members:}

Prof. dr. D. W. P. Thomas (University of Nottingham)

Prof. dr. F. Grassi (Polytechnic University of Milan)

Prof. dr. A. J. M. Pemen (Eindhoven University of Technology)

Dr. ir. R. Serra (Eindhoven University of Technology)

\section{Referee:}

Dr. H. Schippers (Royal Netherlands Aerospace Centre)

The research described in this thesis was carried out in the Power electronics and Electromagnetic Compatibility Group, which is part of the Faculty of Electrical Engineering, Mathematics and Computer Science at the University of Twente, Enschede, the Netherlands.

造任 The author's Ph.D. position was (partly) funded by Royal Netherlands Aerospace Centre

Copyright (c) 2022 by Jesper Lansink Rotgerink

All rights reserved. No part of this publication may be reproduced, stored in a retrieval system, or transmitted, in any form or by any means, electronic, mechanical, photocopying, recording, or otherwise, without the prior written consent of the copyright owner.

ISBN: 978-90-365-5233-2

DOI: $10.3990 / 1.9789036552332$

Cover design by Rens Leerkes

Cover photo by Jesper Lansink Rotgerink

Printed by Gildeprint

Typeset in ${ }_{A} T_{E} X 2 \varepsilon$ 


\title{
CROSSTALK ANALYSIS IN AEROSPACE ENVIRONMENTS
}

\author{
DISSERTATION
}

to obtain

the degree of doctor at the University of Twente, on the authority of the rector magnificus, Prof. dr. ir. A. Veldkamp on account of the decision of the graduation committee, to be publicly defended on Friday $14^{\text {th }}$ January 2022 at $16: 45 h$

by

Jesper Hermanus Gerhardus Johannes Lansink Rotgerink

born on 30th July 1989

in Hengelo, the Netherlands 
This dissertation has been approved by:

The Promoter:

Prof. dr. ir. F. B. J. Leferink 


\section{Summary}

Aviation industry is highly focused on the development of emission-free aircraft. One of the pillars towards achieving this is the More Electric Aircraft (MEA) concept, which should ideally include full electric propulsion in the upcoming decades. Innovations have been driven towards replacement of conventional systems by electrical alternatives, resulting in hundreds of kilometres of on-board wiring and as a consequence an increased risk of malfunction due to undesired electromagnetic coupling - crosstalk. More than ever, this calls for optimisations in the weight of an Electrical Wiring Interconnection System (EWIS), while keeping compliance with safety and Electromagnetic Compatibility (EMC) regulations.

Multiconductor Transmission Line (MTL) models, including all its extensions, or even commercially available full-wave solvers based on for instance Method of Moments (MoM), are suitable for making predictions of crosstalk in complicated cabling systems. However, their significantly high computation times prevent them from applications in which a high variety of realisations has to be computed, such as EWIS optimisations and early risk assessments. Therefore, this thesis focuses on the derivation of methods able to provide efficient estimations of crosstalk that will be of use in early design stages of a EWIS.

The thesis starts with the introduction of a method for low-frequency approximations to the MTL equations. This method is used to derive closed-form expressions for crosstalk between wire pairs with and without a nearby perfectly conducting ground plane. Such expressions clearly relate all designable parameters to crosstalk, which can be used as design rules to make early decisions about routing and segregation of low-risk signals, or early identification of high risks. Moreover, the low-frequency approximations are further applicable to greatly reduce computation times for simulations that involve more complicated non-uniform transmission lines.

One of the measures to protect cabling from crosstalk or external field effects is to apply shielding. However, excessive shielding will result in an unnecessary increase in weight of a EWIS. Consequently, shielding provides opportunity for trade-off and optimisation in terms of EMC protection versus weight. Therefore, the third chapter of this thesis derives generic prediction of crosstalk in cases that involve shielded cables. Clear distinctions are made between regions of various frequency behaviour, and the transition frequencies as well 
as crosstalk levels of each region are again related to all designable parameters.

On top of carrying many electr(on)ic systems, modern aircraft also exhibit a huge change in the materials used. Nowadays, roughly $50 \%$ of aircraft comprise composite materials, such as Carbon-fibre Reinforced Plastics (CFRP), which have a lower conductivity than the conventional aluminium. Therefore, this thesis provides two methods to incorporate the effects of lossy ground planes into the MTL equations. These methods are compared against fullwave simulation results as well as measured crosstalk for the cases of crosstalk between wire pairs above a single lossy ground plane and in between two lossy ground planes. The latter can be regarded as a step towards transmission line modelling of embedded or integrated wiring.

Finally, exact representation of a complex EWIS in aircraft seems utterly impossible. Uncertainties in for instance input data and cabling geometry prevents us from making exact predictions of crosstalk. Design rules will help in making decisions in the early stages of EMC risk assessment. Moreover, sensitivity analyses can provide insight in the parameters that are most crucial to crosstalk levels, as well as insight into the measures to be taken when further optimisations of the wiring systems in later stages of the design process are required. A final step in this thesis showcases the application of the developed transmission line models to perform efficient sensitivity analyses in cases of crosstalk between twisted pairs in cable bundles, as well as the transfer impedance of braided shields. 


\section{Samenvatting}

De ontwikkeling van emissievrije luchtvaart heeft hoge prioriteit voor de luchtvaartindustrie. Eén van de pijlers richting dit doel is het More Electric Aircraft (MEA) concept, dat in de toekomst idealiter ook van elektrische voortstuwing is voorzien. Innovaties hebben tot dusverre geleid tot de vervanging van conventionele systemen door elektrische alternatieven, wat heeft geresulteerd in honderden kilometers aan bekabeling aan boord van vliegtuigen. De consequentie hiervan is een vergroot risico op storingen door ongewenste elektromagnetische koppeling - overspraak. Meer dan ooit vraagt dit om optimalisaties in het gewicht van een Electrical Wiring Interconnection System (EWIS). Dit mag echter nooit ten koste gaan van veiligheid en Elektromagnetische Compatibiliteit (EMC).

Multiconductor Transmissielijn (MTL) modellen inclusief alle mogelijke uitbreidingen, zijn net als commercieel verkrijgbare simulatiepakketten, gebaseerd op bijvoorbeeld Methods of Moments (MoM), geschikt om voorspellingen te doen van overspraak in complexe kabelsystemen. Echter resulteren zulke methodieken in lange rekentijden, wat een obstakel vormt voor inzet waarbij een groot aantal realisaties doorgerekend moet worden, zoals de optimalisatie van een EWIS en vroegtijdige risico analyses. Daarom focust deze thesis zich op de afleiding van methoden die op een efficiënte manier in staat zijn om schattingen van overspraak te bepalen en die gebruikt kunnen worden in de vroege design stadia van een EWIS.

Deze thesis begint met de introductie van een methode voor de bepaling van laag-frequente benaderingen van de MTL vergelijkingen. Deze methode wordt gebruikt om gesloten expressies voor overspraak tussen draadparen in de vrije ruimte en boven een geleidend grondvlak af te leiden. Zulke expressies laten de relaties tussen overspraak en controleerbare parameters duidelijk zien. Ze kunnen worden gebruikt als zowel ontwerpregels voor beslissingen over routering en segregatie van signalen met lage risico's in de vroege ontwerpstadia, als voor vroege identificatie van hoge risico's. Bovendien zijn de laag-frequente benaderingen toepasbaar om de rekentijden voor simulaties van meer gecompliceerde niet-uniforme transmissielijnen enorm te verminderen.

Eén van de maatregelen om bekabeling te beschermen tegen overspraak of externe effecten, is het toepassen van shielding. Echter, het gebruik van 
buitensporige hoeveelheden shielding zal het gewicht van een EWIS onnodig veel laten toenemen. Daarom vormt shielding een goede kandidaat voor trade-off en optimalisatie wat betreft EMC bescherming versus gewicht. Het derde hoofdstuk van deze thesis leidt daarom generieke voorspellingen van overspraak in gevallen met geshielde kabels af. Duidelijk onderscheid kan worden gemaakt tussen regio's met verschillend frequentiegedrag, waarvan de transitiefrequenties en de overspraakniveaus wederom gerelateerd kunnen worden aan alle controleerbare parameters van de kabels.

Naast het toenemende aantal elektrische systemen aan boord, bestaan moderne vliegtuigen ook uit steeds meer nieuwe structurele materialen. Tegenwoordig wordt grofweg $50 \%$ van een vliegtuig gemaakt van composieten zoals Carbon-Fibre Reinforced Plastics (CFRP). Deze materialen hebben een lagere geleiding dan het bijvoorbeeld aluminium. Daarom introduceert hoofdstuk 4 van deze thesis twee methoden om de effecten van grondvlakken met verliezen op te nemen in de MTL vergelijkingen. Deze methoden worden vergeleken met resultaten van commerciële simulatiesoftware en metingen voor overspraak tussen draadparen boven een enkel grondvlak en tussen twee grondvlakken in. Dit laatste kan worden gezien als een stap richting modellering van geïntegreerde of embedded bekabeling.

Exacte representatie van een complexe EWIS in een vliegtuig lijkt simpelweg onmogelijk. Onzekerheden in bijvoorbeeld de inputdata en in de geometrie van kabels weerhoudt ons ervan om overspraak exact te kunnen voorspellen. Ontwerpregels zullen helpen in het maken van beslissingen in de vroege stadia van EMC risico analyses. Daarnaast zijn ook gevoeligheidsanalyses zeer waardevol om inzicht te verkrijgen in welke parameters overspraak het meest beïnvloeden, of welke beschermingsmaatregelen genomen moeten worden als optimalisatie in de verdere stadia van het design nodig zijn. Een laatste stap in deze thesis laat de toepasbaarheid van de ontwikkelde transmissielijnmodellen in een efficiënte gevoeligheidsanalyse zien, waarbij gekeken wordt naar overspraak tussen getwiste draadparen in bundels en de transfer impedantie van kabelshields. 


\section{Contents}

\section{Summary}

Samenvatting

vii

1 Introduction 1

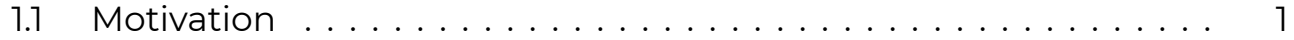

1.2 Thesis goals ......................... 3

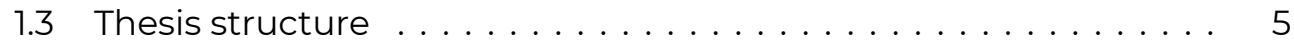

2 Crosstalk analysis for early risk assessment 7

2.1 Multiconductor transmission line equations . . . . . . . . . . 8

2.1.1 Low-frequency approximations . . . . . . . . . . . . . . 10

2.2 Application of the presented method . . . . . . . . . . . 12

2.2.1 Two wire pairs above a perfectly conducting ground plane $\quad 12$

2.2.2 Two wire pairs without nearby ground plane . . . . . . . 20

2.2 .3 Parameter dependencies ................ 22

2.3 Non-uniform transmission lines . . . . . . . . . . . . . . . . 24

2.4 Summary ............................. 25

3 Shielded cables 27

3.1 Transmission line characteristics . . . . . . . . . . . . . . . . . . . . . 29

3.1.1 Per-Unit-Length parameters . . . . . . . . . . . . . . . . 29

3.1 .2 Impedance matrices . . . . . . . . . . . . . . . 32

3.2 Near-end crosstalk . . . . . . . . . . . . . . . . . . . . . 32

3.2.1 One double shielded versus one unshielded wire . . . . . 32

3.2.2 Comparison to literature: one single shielded wire . . . . 34

3.3 Generic crosstalk predictions . . . . . . . . . . . . . 34

3.3.1 One double shielded versus one unshielded wire . . . . . 36

3.3.2 One single shielded versus one unshielded wire . . . . . . 38

3.3.3 Two single shielded wires . . . . . . . . . . . . . . . . 40

3.3.4 Comparison of various shielding configurations . . . . . . 42

3.4 Summary . . . . . . . . . . . . . . . . . . . . . . . . . . . . . . . 44

12
12
24
25


4 Cables in the presence of composite ground planes 45

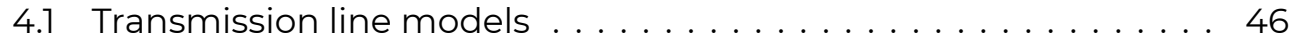

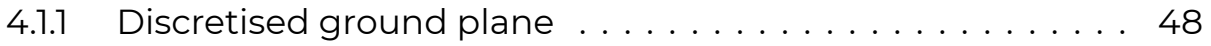

4.1 .2 Ground impedance . . . . . . . . . . . . . . . . . 51

4.2 Results .......................... 52

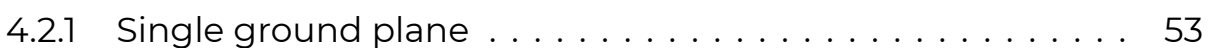

4.2 .2 Double ground plane . . . . . . . . . . . . . 58

4.3 Summary ............................ 61

5 Applications of crosstalk models $\quad 63$

5.1 Twisted pairs in a bundle . . . . . . . . . . . . . . . . 67

5.1 .1 Cable modelling . . . . . . . . . . . . . . . . 67

5.1 .2 Sensitivity simulation results . . . . . . . . . . . . 69

5.1.3 Influence of bundle twist and meandering cables . . . . . 72

5.2 Transfer impedance . . . . . . . . . . . . . . . . . . . . 75

5.2.1 Transmission line model . . . . . . . . . . . . . . . . . . 77

5.2 .2 Transfer impedance computation . . . . . . . . . . . 80

5.2.3 The effects of dielectrics and mismatches . . . . . . . . 83

5.3 Summary .......................... 88

6 Conclusions and future research 93

$\begin{array}{llr}7 & \text { Acknowledgements } & 97\end{array}$

8 Biography 101

$\begin{array}{ll}\text { References } & 102\end{array}$

List of my Publications 111 


\section{Acronyms}

BEATRICS BEtter Analysis of TRansfer Impedance of Cable Shields

CFRP Carbon-fibre Reinforced Plastics

CM Common-mode

DC Direct Current

DM Differential-mode

EM Electromagnetic

EMC Electromagnetic Compatibility

EWIS Electrical Wiring Interconnection System

FEXT Far-end Crosstalk

HIRF High Intensity Radiated Fields

MC Monte Carlo

MEA More Electric Aircraft

MoM Method of Moments

MTL Multiconductor Transmission Line

NEXT Near-end Crosstalk

p.u.l. per-unit-length

PEC Perfectly Electric Conducting

RDSI Random Displacement Spline Interpolation 
Acronyms

SROM Stochastic Reduced Order Model

TL Transmission Line

tpm twists per metre

TWP Twisted Wire Pair

UCS Uniform Cascased Sections 


\section{$1 \mid$ Introduction}

\subsection{Motivation}

Sustainable aviation is one of the major keywords controlling innovations and developments in modern aircraft in the twenty-first century. The European Union's Flightpath 2050 demands from aviation industry a $75 \%$ reduction in $\mathrm{CO}_{2}$ emissions per passenger kilometre and a 90\% reduction in $\mathrm{NO}_{x}$ emissions [1]. This pushes the development of more efficient technologies onboard aircraft. The More Electric Aircraft (MEA) is one of the promising concepts to greatly decrease fuel consumptions and $\mathrm{CO}_{2}$ and $\mathrm{NO}_{x}$ emissions on short term. In a MEA more and more systems are electrically driven. For instance, the application of smart wings will introduce many electronic systems in the wing area [2]. Moreover, on traditional commercial platforms, the Environmental Control System (ECS), the wing Ice Protection System (IPS) and pneumatic systems for actuation of flight control surfaces use hot air bled from the engine to power the systems. On a MEA platform these systems are electrically driven (see Figure 1.1). Last but not least, developments towards emission-free aviation are also moving towards hybrid or full electrification of the aircraft propulsion system.

All these technology developments have greatly increased the amount of electrical wiring in the past decades, while the space for wiring in many parts of the aircraft generally reduces. To illustrate, in 1984 a Boeing 767-200ER carried $120 \mathrm{~km}$ of wiring, while a modern Boeing 787 carries more than 4 times that amount [4]. With future aircraft even more cabling can be expected, which highlights the desire for an Electrical Wiring Interconnection System (EWIS) on-board aircraft that is optimised in terms of weight, volume and costs, while keeping compliance with safety and Electromagnetic Compatibility (EMC) regulations.

Signals applied to closely spaced cables can cause the phenomenon of undesired Electromagnetic (EM) coupling, which is called crosstalk. As MEA will implement a significantly high number of power electronic converters and sensors, crosstalk between cables is an essential topic. Interference by crosstalk might lead to malfunction of systems that are attached to those cables when the interference level is high or when the attached systems are highly 


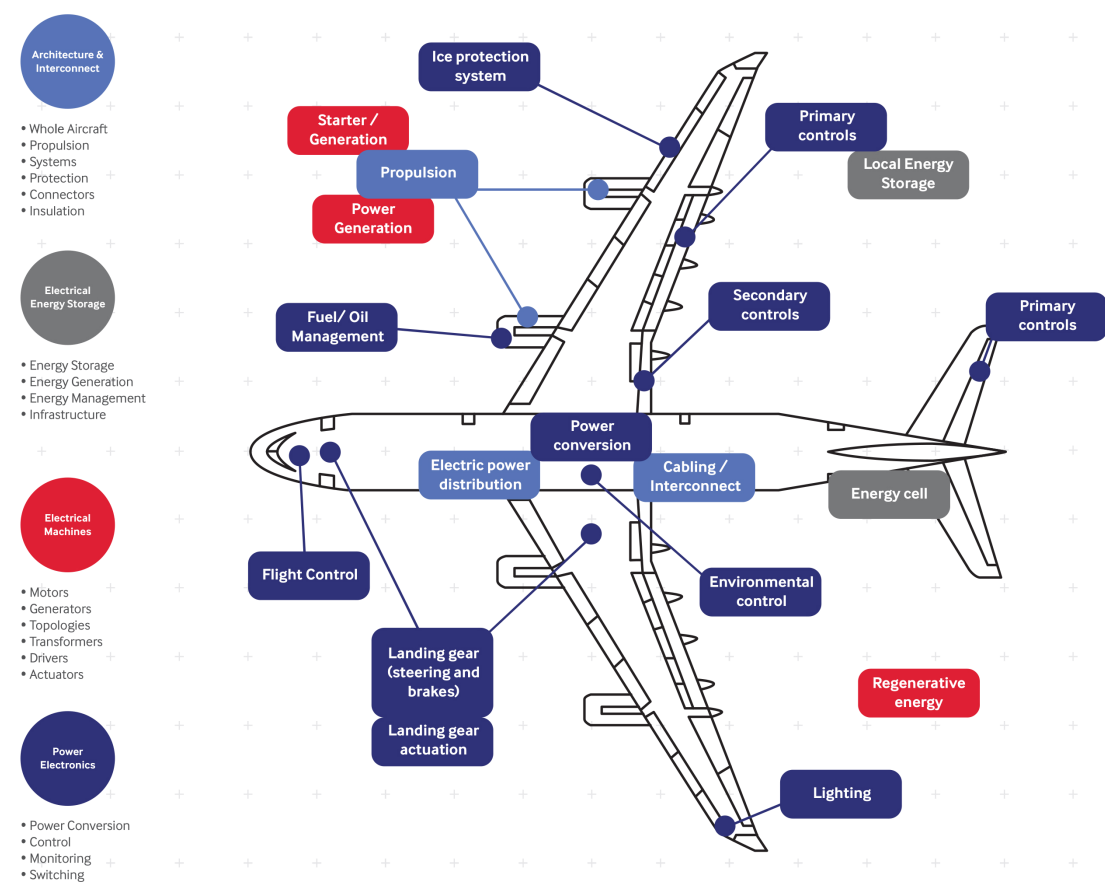

Figure 1.1: Key aircraft subsystems that are subject to electrification in the More Electric Aircraft (source [3]).

sensitive. Trade-offs have to be made between protection measures such as segregation of cables and the application of extra shielding on the one hand, and the extra weight the aircraft has to carry due to such precautions on the other hand. EM simulations can assist in making such trade-offs. Complex, state-of-the-art solution methods are used to simulate crosstalk effects in detailed models of practical cable bundles. Several extensions of the elegant Multiconductor Transmission Line (MTL) theory by Clayton Paul [5] facilitate for example in accurate simulation of twisted wire pairs [6], [7], and the inclusion of inhomogeneous insulation around conductors by numerical estimation of per-unit-length (p.u.l.) parameters [5], [8]. Other advancements make it possible to include more complex longitudinal non-uniformities that are present in practical cable bundles, such as meandering of cables [9], [10] and twist irregularities [11], [12]. Finally, statistical methods could be considered if uncertainties in cable bundles should be taken into account [13-18]. A combination of the necessary state-of-the-art models could yield reliable crosstalk predictions in complex cable bundles.

However, with modern aircraft carrying several hundred kilometres of cabling, performing such complete and accurate simulations for every possible configuration of an entire EWIS will be a computationally huge task, even 
when quick simulation methods are used for the computation of a single EWIS [19]. Especially when used in optimisation of cable bundles, in which numerous realisations of cable bundles and routing scenarios should be analysed, more efficient methods are useful to reduce the solution space. Therefore, the effective application of EMC design rules for cable routing is important, since these allow early identification of possible risks. The dependencies of crosstalk on designable parameters such as separation distances are essential for such design rules. Designable parameters are those of which the values can be changed by the design engineer. Quick analysis of the entire EWIS based upon such design rules can yield both routing for low-risk signals and EMC bottle-necks for the high-risk signals. Subsequent design stages could then involve accurate assessment of the routing of these high-risk signals. The desired design rules can be obtained by simplified crosstalk expressions as a result of low-frequency analysis of the MTL.

Apart from the trends of ever increasing amounts of on-board cabling, modern aircraft structures are more and more composite-based (see Figure 1.2). Today's commercial aircraft, such as the Airbus A350 and Boeing 787, comprise about $50 \%$ of its weight in composites [20,21]. From a crosstalk perspective it is well-known that it is good practice to place cables close to a good conducting surface, as this generally reduces crosstalk levels $[22,23]$. However, since these modern aircraft carry so much lossy composite materials, there is a need to model the effects on crosstalk behaviour due to replacement of metal parts in aircraft by less conducting material such as Carbonfibre Reinforced Plastics (CFRP). Efficient simulation models for crosstalk in cable bundles that can include the lossy properties of CFRP ground planes are a requirement for modern aircraft EWIS design. One of the challenging aspects in the use of composite materials is the large uncertainty in conductivity between the various layers. However, for the crosstalk analysis in this thesis representation of the CFRP by an isotropic plane with equivalent conductivity is sufficient $[24,25]$.

\subsection{Thesis goals}

The goal of this thesis is to develop efficient methods for analysis of crosstalk that provide quick estimations of crosstalk levels or sensitivities. Such methods can give an engineer quick insight into crosstalk phenomena for a large variety of EWIS realisations in modern aircraft, facilitating both early risk assessment as well as EWIS optimisations. To reach this goal, a bottom-up approach leads from closed-form expressions for crosstalk in the very simple case of unshielded and untwisted wire pairs, through the inclusion of shields and lossy grounds. Finally, MTL models are used to perform sensitivity analyses of more complex cabling phenomena such as cable twist and meandering, as well as transfer impedance of braided shields. Crosstalk appears not only in cables, but also in for instance connectors or through pigtails [27-30]. 


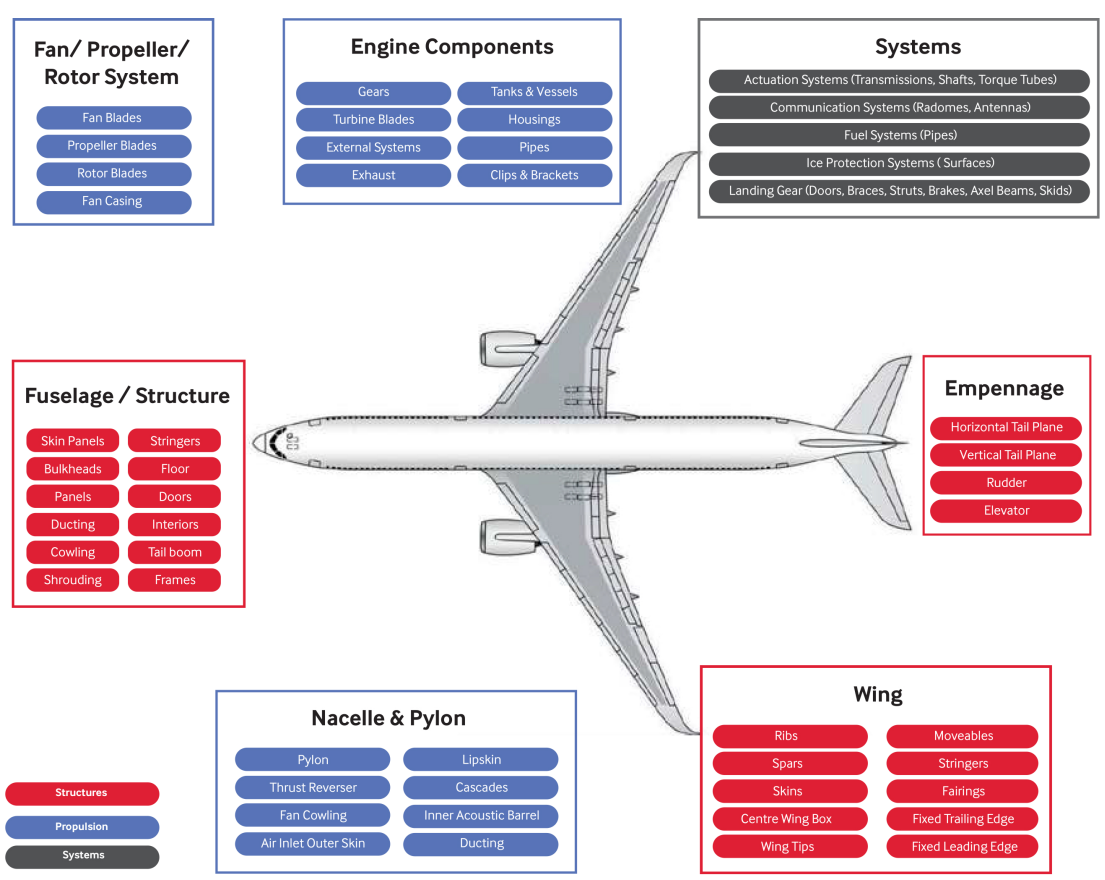

Figure 1.2: Illustration of the composite products in current aicraft (source [26]).

Even though the MTL models used in this thesis are well applicable to such cases, the scope of this thesis is restricted to cables. Specifically, MTL models are utilised for the following situations:

- Closed-form expressions for crosstalk between unshielded, untwisted wire pairs that yield direct relations between crosstalk and designable parameters.

- Generic predictions of crosstalk between cables that include shielding, which clearly identify the various regions of frequency behaviour introduced by shielding.

- Analysis of the effects to crosstalk behaviour of replacing metallic ground planes by lossy materials such as CFRP.

- Application of the transmission line models to perform sensitivity analysis to crosstalk in more complex cable bundles and transfer impedance measurement methods. 


\subsection{Thesis structure}

The structure of this thesis reflects the bottom-up approach of crosstalk analysis by the use of the MTL models as mentioned in the previous section. That is, the thesis starts in Chapter 2 with the analysis of crosstalk in one of the simpler cabling configurations possible. It is shown that by using low-frequency approximations to the MTL equations closed-form expressions can be derived that clearly indicate the dependencies on all cabling parameters. This yields useful insight in crosstalk phenomena and the measures that can be taken for early risk assessment in the optimisation of cable bundles. Moreover, it is shown that these low-frequency approximations can also be used to greatly reduce computation times for crosstalk between twisted wire pairs. This is later applied in sensitivity analyses in Chapter 5.

One layer of complexity is added in the form of shielding in Chapter 3. The methods to include one or two layers of shielding to a cable in an MTL model are introduced, after which again low-frequency analysis yields expressions that clearly explain the frequency behaviour of crosstalk that is caused by the braided shields. Further simplification of these low-frequency expressions into specified frequency regions results in generic predictions of crosstalk in cases that involve shielded wires. These generic predictions clearly relate the crosstalk to all shielding parameters.

The influences of lossy ground planes, such as CFRP, in the vicinity of cabling is analysed in Chapter 4. Two ways of incorporating such lossy ground planes with finite thickness into MTL modelling are introduced and applied to the case of crosstalk between two wire pairs above one CFRP ground plane, and between two CFRP ground planes. Comparing such results to the cases of aluminium ground planes or cabling without nearby ground planes yields interesting conclusions on frequency behaviour. Moreover, the two newly introduced methods reach significant speed-ups compared to full-wave Method of Moments (MoM) simulations.

In Chapter 5 MTL methods are applied in sensitivity analyses. Firstly, the sensitivity with respect to model parameters of crosstalk between twisted wire pairs in bundles that include bundle twist and cable meandering is analysed. Secondly, MTL models of transfer impedance measurement methods are introduced and used to investigate the sensitivities of these methods to termination mismatches and dielectric permittivity.

Each of the Chapters contains a short summary of results in the closing section, while Chapter 6 provides the overall conclusions to this thesis, including directions for further research. 
CHAPTER 7. INTRODUCTION

$\oplus$ $\emptyset$ 


\section{$2 \|$ Crosstalk analysis for early risk assessment}

Chapter 1 introduced the benefits of simplified analytical crosstalk expressions for early risk assessment, when compared to for instance complex numerical simulation methods that take into account every detail of the cabling configuration. Low-frequency analysis of the MTL models, i.e. for electrically short lines, will be used in this chapter, which is based on [22], to derive such expressions.

Crosstalk for low frequencies, or electrically short lines, in a lossless case can quickly be computed by Spice simulations. However, these do not result in clear dependencies of crosstalk with respect to designable parameters. Alternatively, circuit analysis of lossless and electrically short Transmission Lines (TLS) is often used to obtain analytic crosstalk expressions. For instance, White derives expressions for the coupling between two single wires above ground [31] and in [32] and [33] Paul also derives similar expressions for crosstalk between a single wire and a twisted pair or a shielded wire above ground. However, circuit analysis becomes tedious for more than 2 or 3 wires above a ground plane, and therefore there is a lack of such analytic expressions in literature.

In this chapter we present a mathematical method to derive simplified but efficient closed-form crosstalk expressions directly from the matrix formulations of the MTL equations. These expressions directly relate crosstalk to all designable parameters and can be used for design rules to make early decisions about routing and segregation of low-risk signals. Application of this method results in such crosstalk expressions for four and five-conductor TLs involving wire pairs 1) above a perfectly conducting ground plane, and 2) without nearby ground plane. A first application of this method, published in [23], showed fourth-order dependence with respect to separation distance for crosstalk between wire pairs close to a perfectly conducting ground plane. In this chapter, the method is also applied to crosstalk between wire pairs where a ground plane is absent. The derived closed-form expressions for both situations clearly show the differences in dependencies of crosstalk on all designable parameters. Finally, it is also shown that the set of low-frequency 
equations that form the basis of the presented methodology is very useful for a quick, lossless crosstalk analysis of more complex and non-uniform MTLs. A speedup of more than 20 times is reached when compared to the global MTL method. The methods and expressions in this chapter also form an efficient candidate for multiple run optimisation of an entire EWIS. This will be shown by the application of these low-frequency equations in the sensitivity analysis to more complicated cable bundles in Chapter 5.

Section 2.1 introduces the mathematical context of the low-frequency method. In Section 2.1.1 two examples are given, comprising two wire pairs 1) close to a perfect ground plane and 2) without nearby ground plane. Closed-form expressions are derived and used for the analysis of parameter sensitivity. The final section presents a summary of the findings in this chapter.

\subsection{Multiconductor transmission line equations}

Consider an MTL with $n+1$ conductors, including a reference conductor. Broadly used simulation methods to analyse crosstalk between cables are based on the MTL equations [5]:

$$
\begin{aligned}
\frac{d}{d z} \mathbf{V}(z) & =-\hat{\mathbf{Z}} \mathbf{I}(z), \\
\frac{d}{d z} \mathbf{I}(z) & =-\hat{\mathbf{Y}} \mathbf{V}(z) .
\end{aligned}
$$

In these equations $\mathbf{V}$ and $\mathbf{I}$ are $n$-dimensional vectors representing the voltages and currents in each of the $n$ non-reference conductors of the MTL along its propagation direction $z$. For frequency domain solutions, the p.u.l. impedance matrix $\hat{\mathbf{Z}}$ and admittance matrix $\hat{\mathbf{Y}}$ are usually defined by:

$$
\begin{aligned}
& \hat{\mathbf{Z}}=\mathbf{R}+j \omega \mathbf{L} \\
& \hat{\mathbf{Y}}=\mathbf{G}+j \omega \mathbf{C} .
\end{aligned}
$$

Here $\boldsymbol{\omega}$ is the angular frequency of the signal travelling down the transmission line. The resistance matrix $\mathbf{R}$, the capacitance matrix $\mathbf{C}$, the inductance matrix $\mathbf{L}$ and the conductance matrix $\mathbf{G}$, together form the p.u.l. parameters, which contain all cross-sectional information of the MTL.

Let $\mathbf{V}_{0}$ denote the vector containing the voltages at the near-end of the $n$ non-reference conductors with respect to the reference. Corresponding currents are represented by $\mathbf{I}_{0}$. Since crosstalk is defined as the ratio of voltage induced in a receptor or victim TL, over that in the generator or culprit line, the Near-end Crosstalk (NEXT) $\gamma_{N E}$ can be expressed by:

$$
\gamma_{N E}=\frac{\mathbf{U}_{2}^{T} \mathbf{v}_{0}}{\mathbf{U}_{1}^{T} \mathbf{v}_{0}}
$$




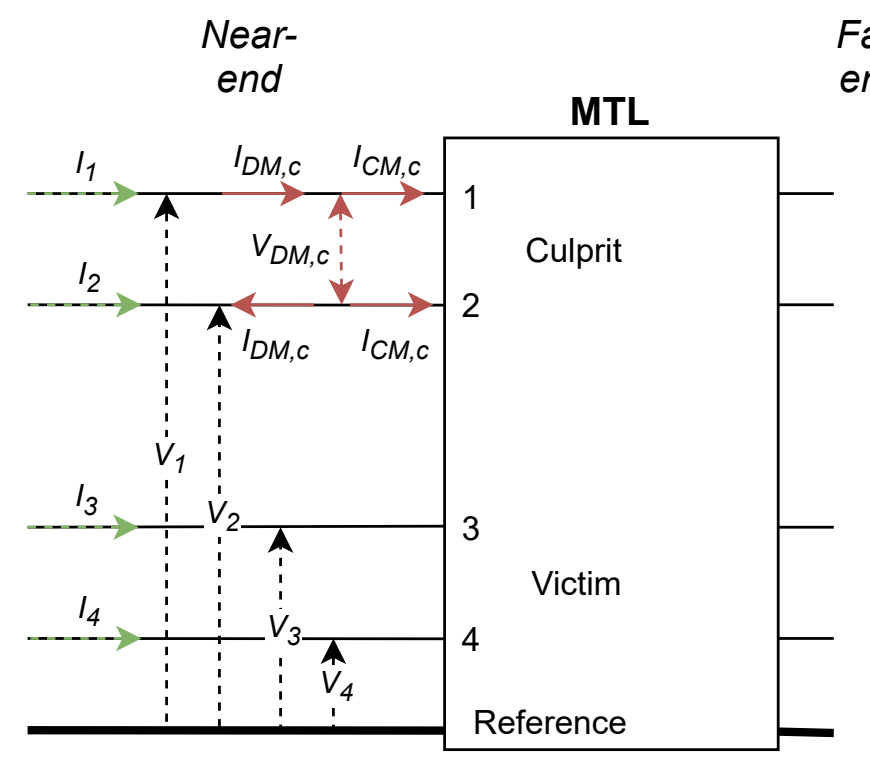

Far-

end

Figure 2.1: Representation of the MTL as a four-port network, in which the numbering of currents and voltages is illustrated. For the culprit, the DM currents $\left(I_{D M}, c\right)$ and voltage $\left(V_{D M, C}\right)$ are also shown, as well as the CM currents $\left(I_{C M, C}\right)$.

Here $\mathbf{U}_{1}$ and $\mathbf{U}_{2}$ are the $n$-dimensional vectors that select the necessary voltages from $\mathbf{V}_{0}$ to obtain either Common-mode (CM) or Differential-mode (DM) voltages of victim and culprit in the numerator and denominator of (2.3), respectively. For example, in case DM crosstalk is required, from Figure 2.1 it can be derived that $\mathbf{U}_{1}=(-1,1,0,0)$ and $\mathbf{U}_{2}=(0,0,-1,1)$.

To obtain closed-form NEXT expressions, the vector of voltages at near-end is required. For Far-end Crosstalk (FEXT) the far-end voltage is used in (2.3), but the analysis is completely similar. Therefore, in this chapter we discuss only NEXT. For the analysis of MTLs, matrix equations are presented in [5]. One way of solving these MTL equations involves the use of chain parameter matrices $\boldsymbol{\Phi}$, which can be used to relate the voltages and currents at the beginning of the TL to the end of the TL:

$$
\left[\begin{array}{l}
\mathbf{V}_{\ell} \\
\mathbf{I}_{\ell}
\end{array}\right]=\left[\begin{array}{ll}
\boldsymbol{\Phi}_{11} & \boldsymbol{\Phi}_{12} \\
\boldsymbol{\Phi}_{21} & \boldsymbol{\Phi}_{22}
\end{array}\right]\left[\begin{array}{l}
\mathbf{V}_{0} \\
\mathbf{I}_{0}
\end{array}\right]
$$

Suppose that the terminations of the MTL can be represented by a Thévenin equivalent, then the boundary condition for the near-end voltages is expressed as:

$$
\mathbf{v}_{0}=\mathbf{V}_{S}-\mathbf{Z}_{S} \mathbf{I}_{0}
$$

Here $\mathbf{V}_{S}$ is the vector of voltage sources at the near-end side, and the termination networks at the near and far-end sides are represented by the im- 
pedance matrices $\mathbf{Z}_{S}$ and $\mathbf{Z}_{L}$. If there are any additional voltage sources at the far-end side they are contained in a vector given by $\mathbf{V}_{L}$. In this case, the desired voltages at the near-end can be obtained by solving the corresponding currents from:

$$
\begin{aligned}
\mathbf{A I}_{0} & =\left[\boldsymbol{\Phi}_{11}-\mathbf{Z}_{L} \boldsymbol{\Phi}_{21}\right] \mathbf{V}_{S}-\mathbf{V}_{L} \\
\mathbf{A} & =\left[\boldsymbol{\Phi}_{11} \mathbf{Z}_{S}+\mathbf{Z}_{L} \boldsymbol{\Phi}_{22}-\boldsymbol{\Phi}_{12}-\mathbf{Z}_{L} \boldsymbol{\Phi}_{21} \mathbf{Z}_{S}\right]
\end{aligned}
$$

and substituting $\mathbf{I}_{0}$ into (2.5). For crosstalk analysis, we assume presence of one voltage source at the near-end side, implying:

$$
\mathbf{V}_{S}=V_{S} \mathbf{U}_{1}, \quad \mathbf{V}_{L}=\mathbf{0},
$$

in which $V_{S}$ controls the magnitude of the voltage source.

Alternatively, suppose that the terminations are given by a Norton equivalent representation. Therefore, the terminations are represented by admittance matrices $\mathbf{Y}_{S}$ and $\mathbf{Y}_{L}$ on source and load side. Then the near-end voltages can be solved from:

$$
\begin{aligned}
\mathbf{A} \mathbf{V}_{0} & =\left[\boldsymbol{\Phi}_{22}-\mathbf{Y}_{L} \boldsymbol{\Phi}_{12}\right] \mathbf{I}_{S}+\mathbf{I}_{L} \\
\mathbf{A} & =\left[\boldsymbol{\Phi}_{22} \mathbf{Y}_{S}+\mathbf{Y}_{L} \boldsymbol{\Phi}_{11}-\boldsymbol{\Phi}_{21}-\mathbf{Y}_{L} \boldsymbol{\Phi}_{12} \mathbf{Y}_{S}\right]
\end{aligned}
$$

For crosstalk analysis we now assume one current source at the near-end side, implying:

$$
\mathbf{I}_{S}=I_{S} \mathbf{U}_{1}, \quad \mathbf{I}_{L}=\mathbf{0},
$$

in which $I_{S}$ controls the magnitude of the voltage source.

\subsubsection{Low-frequency approximations}

Expressions that clearly give relations between crosstalk and designable parameters can be obtained by using low-frequency approximations. Such approximations are usually accurate up to frequencies at which the electrical length of the TL is short with respect to the wavelength of the transmitted signals. At such frequencies the chain parameters can be approximated by:

$$
\begin{aligned}
& \boldsymbol{\Phi}_{11}=\boldsymbol{\Phi}_{22}=\mathbf{I}_{n} \\
& \boldsymbol{\Phi}_{12}=-\mathrm{j} \omega \ell \mathbf{L}-\ell \mathbf{R} \\
& \boldsymbol{\Phi}_{21}=-\mathrm{j} \omega \ell \mathbf{C}-\ell \mathbf{G},
\end{aligned}
$$

where $\mathbf{I}_{n}$ is the $n$-dimensional identity matrix and $\ell$ the length of the line. Dielectric losses and conductor losses can be included in the p.u.l. conductance matrix $\mathbf{G}$ and resistance matrix $\mathbf{R}$, respectively. However, by the low-frequency technique presented in this chapter the high-frequency effects of dielectric and conductor losses to crosstalk will be neglected. Low-frequency effects of 
common-impedance coupling can be derived in a way similar to how inductive and capacitive coupling will be computed for the cases in Section 2.1.1. However, these examples consider crosstalk between wire pairs in which case there is no common return conductor. Therefore, in this chapter we neglect conductor losses, by which $\mathbf{R}=\mathbf{0}$. The losses in dielectric media are neglected in this entire thesis, implying $\mathbf{G}=\mathbf{0}$. Moreover, the analysis in this chapter presumes weak coupling. Therefore, the usually small secondary coupling effects, as well as proximity effects, which are small whenever the wires of a wire pair are separated by isolation, are both neglected.

Substitution of (2.10) in (2.6) or (2.8) gives the basis for the low-frequency crosstalk analysis. The matrix inversion of $\mathbf{A}$, needed for the computation of voltages in all conductors, can be approximated by a Taylor expansion for low frequencies. Therefore, $\mathbf{A}$ is manipulated to be in the following form:

$$
\mathbf{A}=\mathbf{I}_{n}+\mathrm{j} \omega \ell \mathbf{B} .
$$

Here, for the Thévenin representation, B follows from substitution of (2.10) into (2.6) and multiplication by $\tilde{\mathbf{Z}}=\left[\mathbf{Z}_{S}+\mathbf{Z}_{L}\right]^{-1}$. For the Norton representation we multiply by $\tilde{\mathbf{Y}}=\left[\mathbf{Y}_{S}+\mathbf{Y}_{L}\right]^{-1}$ after substitution of (2.10) into (2.8). For all frequencies such that $\omega \ell\|\mathbf{B}\| \ll 1$, which usually holds for electrically short lines, the inversion is then approximated by:

$$
\mathbf{A}^{-1}=\mathbf{I}_{n}-\mathrm{j} \omega \ell \mathbf{B} .
$$

We apply this operation to (2.6) (after substitution of (2.10)) to obtain the vector of currents at the near-end side. The vector of near-end voltages is then computed by (2.5), resulting in:

$$
\mathbf{V}_{0} \approx V_{S} \mathbf{U}_{1}-V_{s} \mathbf{Z}_{S} \tilde{\mathbf{z}} \mathbf{U}_{1}+\mathrm{j} \omega \ell V_{S} \mathbf{Z}_{S}\left[\tilde{\mathbf{z}} \mathbf{L} \tilde{\mathbf{z}}+\tilde{\mathbf{z}} \mathbf{Z}_{L} \mathbf{C} \mathbf{Z}_{S} \tilde{\mathbf{z}}-\tilde{\mathbf{z}} \mathbf{Z}_{L} \mathbf{C}\right] \mathbf{U}_{1}
$$

If the termination networks on both sides of the transmission line are equal, i.e. $\mathbf{Z}_{S}=\mathbf{Z}_{L}=\mathbf{Z}$, then (2.13) simplifies to:

$$
\mathbf{v}_{0} \approx \frac{V_{S}}{2}\left[\mathbf{I}_{n}+\frac{1}{2} \mathrm{j} \omega \ell\left(\mathbf{L Z}^{-1}-\mathbf{Z C}\right)\right] \mathbf{U}_{1}
$$

Similarly, for the Norton equivalent representation the Taylor approximation is applied to (2.8) to obtain the vector of voltages at the near-end side:

$$
\mathbf{V}_{0} \approx I_{S} \tilde{\mathbf{Y}} \mathbf{U}_{1}+\mathrm{j} \omega \ell I_{S}\left[\tilde{\mathbf{Y}} \mathbf{Y}_{L} \mathbf{L}-\tilde{\mathbf{Y}} \mathbf{C} \tilde{\mathbf{Y}}-\tilde{\mathbf{Y}} \mathbf{Y}_{L} \mathbf{L} \mathbf{Y}_{S} \tilde{\mathbf{Y}}\right] \mathbf{U}_{1}
$$

Again, if the terminations on both sides of the transmission line are equal, i.e. $\mathbf{Y}_{S}=\mathbf{Y}_{L}=\mathbf{Y}$, then (2.15) simplifies to:

$$
\mathbf{V}_{0} \approx \frac{I_{S}}{2}\left[\mathbf{Y}^{-1}+\frac{1}{2} j \omega \ell\left(\mathbf{L}-\mathbf{Y}^{-1} \mathbf{C} \mathbf{Y}^{-1}\right)\right] \mathbf{U}_{1}
$$

Once the vector of voltages in each conductor has been obtained, (2.3) is applied to compute the resulting crosstalk. 
Figure 2.2 gives an example of the NEXT computed between two $1.9 \mathrm{~m}$ long wire pairs with $20 \mathrm{~mm}$ separation, situated parallel at a distance of $1.5 \mathrm{~mm}$ from an infinite ground plane, as shown by the cross-section in Figure 2.3a. The values for all other parameters are given in [23] (and equal to those given later in Section 2.2.1). The results in the figure show that by complete MTL simulation or full-wave Feko [34] simulation a detailed prediction of crosstalk in the frequency domain can be obtained. In the MTL simulations analytic and exact expressions for the chain parameter matrices are used, including all coupling and propagation effects in all conductors. Full-wave simulations take into account all EM interactions, but are much more extensive to set up. By substituting the low-frequency approximations for $\boldsymbol{\Phi}$ in (2.10), direct solution of (2.6) yields a curve in which resonances disappeared, but the trends of crosstalk behaviour in the frequency domain are still present (black line). The red dashed curve represents the low-frequency approximations as result of the analysis in this chapter, which do not give an exact solution for crosstalk behaviour, but are perfect for determining relations to all designable parameters.

\subsection{Application of the presented method}

In this section, for two situations closed-form expressions are derived. In [23], the presented methodology was applied to two single wires above a ground plane. This provided results that are widely reported in literature. In this chapter, the analysis is applied to crosstalk between wire pairs parallel and close to a ground plane, as well as without ground plane. For both cases first a general crosstalk expression is presented, after which leading-order dependency on designable parameters is derived with Taylor expansions after some extra assumptions.

\subsubsection{Two wire pairs above a perfectly conducting ground plane}

Consider the cross-section in Figure 2.3a, comprising two wire pairs parallel to a ground plane. The wires are numbered 1 to 4 from left to right and a differential voltage source is included in the near-end termination of the first pair; differential-mode crosstalk results. The pairs are separated by a distance $d$ and all wires are at equal heights $h$ above the ground plane and of radius $r$. The wires in both pairs have an intra-pair separation distance $a$.

Terminations The terminations for the wire pairs are equal to those used in [23] and shown in Figure 2.4. Norton equivalent representation techniques can be used to obtain the corresponding termination matrices: 


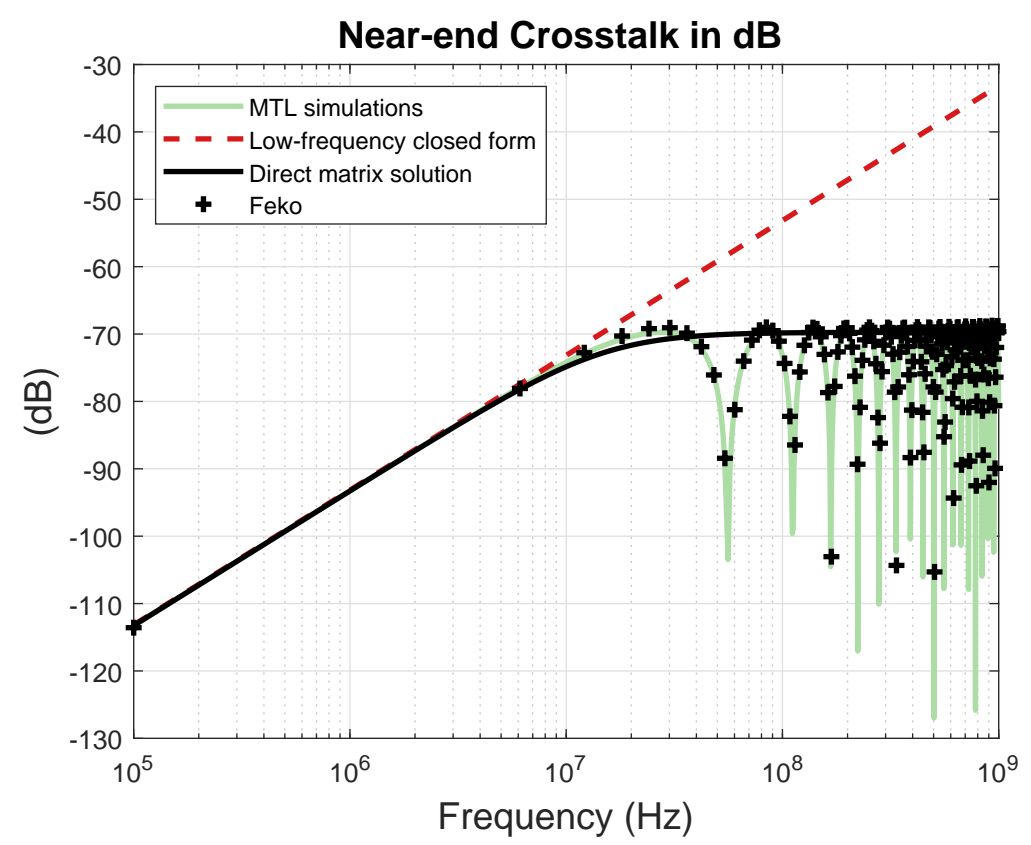

Figure 2.2: MTL (green) and Feko (black crosses) simulations of NEXT between two wire pairs above an infinite ground plane (see Section 2.2.1). The red dashed line shows the low-frequency approximation. The black line is the direct solution found from Paul's matrix equation, given by (2.6), after substitution of (2.10).

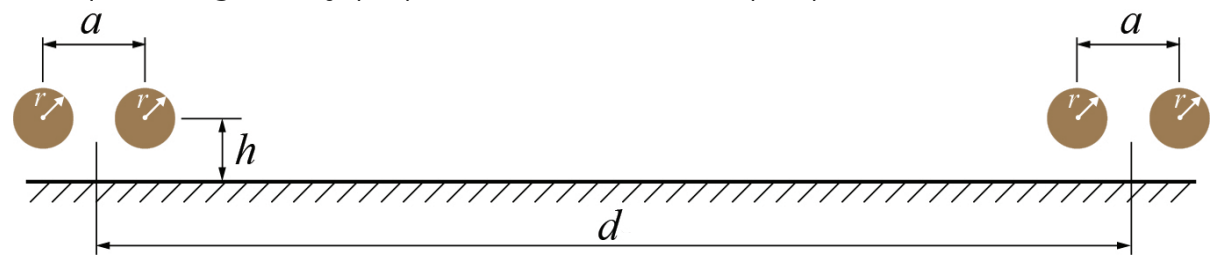

(a) Two wire pairs close to an infinite, perfectly conducting ground plane

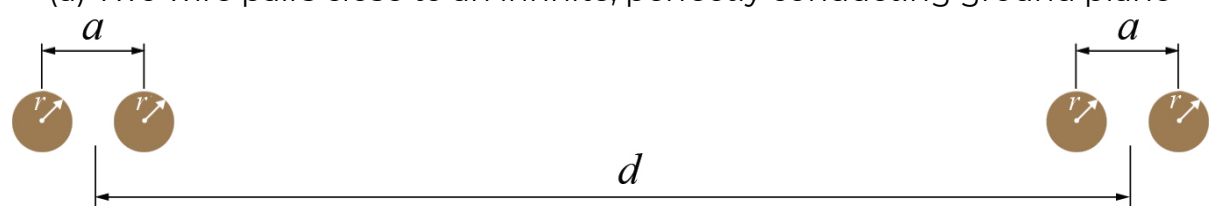

(b) Two wire pairs without nearby ground plane.

Figure 2.3: Illustration of the cross-sections for two cabling configurations analysed in this chapter. 


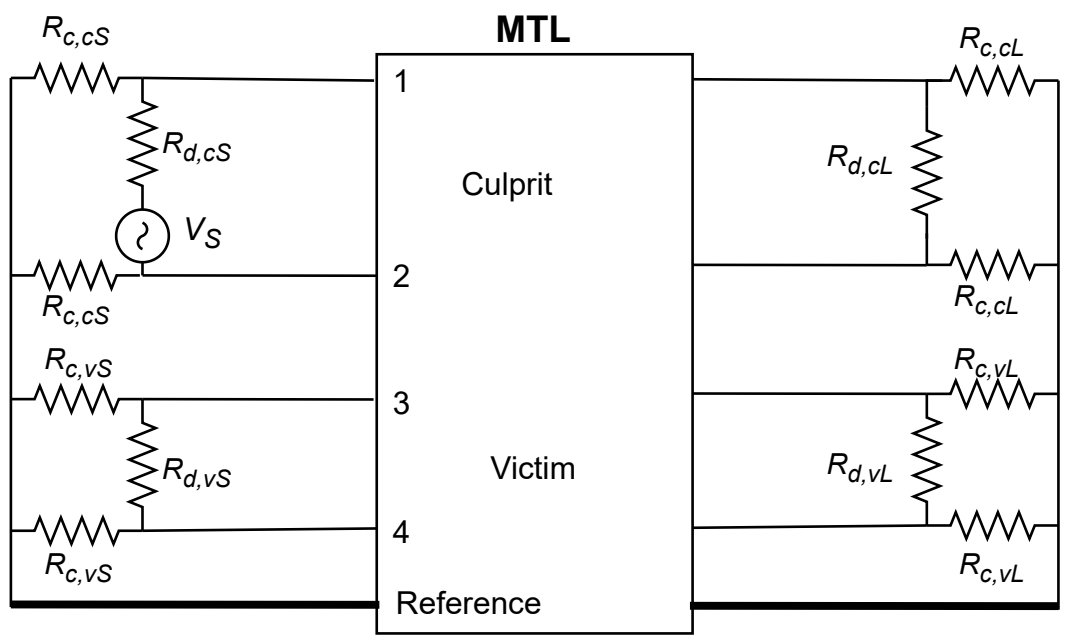

Figure 2.4: Illustration of the termination network for two wire pairs above a ground plane.

$$
\begin{aligned}
\mathbf{z}_{x} & =\left[\begin{array}{cc}
\mathbf{Z}_{c x}^{*} & \mathbf{0} \\
\mathbf{0} & \mathbf{Z}_{v x}^{*}
\end{array}\right] \\
\mathbf{z}_{m x}^{*} & =R_{d, m x}\left[\begin{array}{ll}
c_{m x}+\kappa_{m x} & c_{m x}-\kappa_{m x} \\
c_{m x}-\kappa_{m x} & c_{m x}+\kappa_{m x}
\end{array}\right], m \in\{c, v\} .
\end{aligned}
$$

where subscripts $v$ and $c$ represent the victim or the culprit, while the subscript $X \in\{S, L\}$ indicates the termination on source or load side. Moreover, $\kappa_{m x}=\left[2\left(R_{d, m x} / R_{c, m x}+2\right)\right]^{-1}, c_{m x}=\left[R_{d, m x}\left(R_{d, m x} / R_{c, m x}+2\right) / R_{c, m x}\right]^{-1}+$ $\kappa_{m x}$. Here the variable $R_{d, m x}$ is the DM and $R_{c, m x}$ the CM termination of either culprit or victim wire pair at the load or source side (see also Figure 2.4). The CM impedance $R_{c, m x}$ is present if the wire pair is also connected to ground via a certain impedance. In the measurements presented in this chapter, wire pairs are always connected to ground via a balun with known impedance. Finally, since a DM source is present in the culprit line, we define $\mathbf{U}_{1}=(-1,1,0,0)^{T}$ and $\mathbf{U}_{2}=(0,0,1,1)^{T}$. By (2.5), this implies that a voltage difference of $2 V_{S}$ is set and maintained.

Near-end crosstalk The given termination matrices in (2.17) can be used to compute the numerator and denominator of (2.3): 


$$
\begin{aligned}
\mathbf{U}_{1}^{T} \mathbf{V}_{0} \approx & \frac{2 V_{S} R_{d, c L} K_{c L}}{R_{d, c L} K_{c L}+R_{d, c S} K_{c S}} \\
\mathbf{U}_{2}^{T} \mathbf{V}_{0} \approx & \frac{j \omega l V_{S} R_{d, v S} K_{v S}\left(l_{13}+l_{24}-\left(l_{14}+l_{23}\right)\right)}{2\left(R_{d, c L} K_{c L}+R_{d, c S} K_{c S}\right)\left(R_{d, v L} K_{v L}+R_{d, v S} K_{v S}\right)} \\
& -\frac{2 \mathrm{j} \omega l V_{S} R_{d, c L} R_{d, v L} R_{d, v S} K_{c L} K_{v L} K_{v S}\left(c_{13}+c_{24}-\left(c_{14}+c_{23}\right)\right)}{\left(R_{d, c L} K_{c L}+R_{d, c S} K_{c S}\right)\left(R_{d, v L} K_{v L}+R_{d, v S} K_{v S}\right)}
\end{aligned}
$$

Here, $l_{i j}$ and $c_{i j}$ are elements of the inductance and capacitance matrices. These can be computed by for instance the analytic expressions in [5], or numerical methods [8]. Substituting (2.18) into (2.3) results in the following expressions for capacitive crosstalk:

$$
\gamma_{N E, c a p} \approx \mathrm{j} \omega \ell \frac{R_{d, v L} R_{d, v S} K_{v L} K_{v S}\left(c_{14}+c_{23}-c_{13}-c_{24}\right)}{\left(R_{d, v L} K_{v L}+R_{d, v S} K_{v S}\right)},
$$

and inductive crosstalk:

$$
\gamma_{N E, \text { ind }} \approx \mathrm{j} \omega \ell \frac{R_{d, v S K_{v S}}\left(l_{13}+l_{24}-l_{14}-l_{23}\right)}{4 R_{d, c L} K_{C L}\left(R_{d, v L} K_{v L}+R_{d, v S} K_{v S}\right)} .
$$

In the next subsection Taylor expansions of the analytical p.u.l. parameters will be used to obtain the leading order, closed-form crosstalk expressions.

Closed-form expression Per-unit-length parameters characterise the transmission line. We use Taylor expansions combined with the nice structure of inductance and capacitance matrices to derive our low-frequency closed form expressions for crosstalk. The medium surrounding the wires is assumed to be homogeneous. Material properties are given by $\varepsilon_{r} \varepsilon_{0}$ and $\mu_{r} \mu_{0}$, where the subscripts $r$ and 0 indicate relative and free space permittivity or permeability, respectively. Magnetic materials are unusual in cabling and are therefore not considered for this thesis, by which the relative permeability will be set to one from here on. Paul's analytical expressions for the self-inductance $l_{i i}$ and mutual inductance $l_{i j}$ of cylindrical conductors above a perfectly conducting ground plane are given by [5]:

$$
l_{i i}=\frac{\mu_{0}}{2 \pi} \ln \left(\frac{2 h_{i}}{r_{i}}\right), \quad l_{i j}=\frac{\mu_{0}}{4 \pi} \ln \left(1+\frac{4 h_{i} h_{j}}{d_{i j}^{2}}\right) .
$$

Here $h_{i}$ and $r_{i}$ are the height above ground and wire radius of conductor $i$, while $d_{i j}$ is the separation distance between conductors $i$ and $j$. Applying these equations to the test case of two wire pairs above a ground plane as given by Figure 2.3a, the inductance matrix can be written as:

$$
\mathbf{L}=\left[\begin{array}{cc}
\mathbf{L}_{000} & \mathbf{L}_{12} \\
\mathbf{L}_{12}^{T} & \mathbf{L}_{000}
\end{array}\right]
$$


where $\mathbf{L}_{000}$ represents the inductance within a pair, given by:

$$
\mathbf{L}_{000}=\beta\left[\begin{array}{cc}
\ln (2 h / r) & \ln \sqrt{1+y} \\
\ln \sqrt{1+y} & \ln (2 h / r)
\end{array}\right],
$$

where $\beta=\mu_{0} / 2 \pi$ and $y=4 h^{2} / a^{2}$. The mutual inductance between the pairs equals:

$$
\mathbf{L}_{12}=\frac{\beta}{2}\left[\begin{array}{cc}
\ln (1+x) & \ln \left(1+\frac{x}{(1+\alpha)^{2}}\right) \\
\ln \left(1+\frac{x}{(1-\alpha)^{2}}\right) & \ln (1+x)
\end{array}\right],
$$

where $\alpha=a / d$ and $x=4 h^{2} / d^{2}$. Suppose $\alpha$ is a small parameter, i.e. $a \ll d$. Then the inversion of $\mathbf{L}$, which is necessary for the computation of the capacitance matrix, can be approximated by Taylor expansions. Therefore, firstly $\mathbf{L}_{12}$ is expanded in terms of $\alpha$ :

$$
\hat{\mathbf{L}}_{12}=\underbrace{\left[\begin{array}{ll}
1 & 1 \\
1 & 1
\end{array}\right]}_{\mathbf{L}_{01}} b+\underbrace{\left[\begin{array}{cc}
0 & -1 \\
1 & 0
\end{array}\right] \frac{\beta x}{x+1}}_{\mathbf{L}_{1}} \alpha+\underbrace{\left[\begin{array}{ll}
0 & 1 \\
1 & 0
\end{array}\right] \frac{\beta}{2} \frac{3 x+x^{2}}{(x+1)^{2}}}_{\mathbf{L}_{2}} \alpha^{2},
$$

where $b=\beta / 2 \ln (1+x)$. The hat notation is used for Taylor approximations. Substituting (2.25) into (2.22) yields a second order Taylor approximation for $\mathbf{L}$ :

$$
\begin{aligned}
\hat{\mathbf{L}} & =\underbrace{\mathbf{L}_{00}+\mathbf{L}_{01} b}_{\mathbf{L}_{0}}+\mathbf{L}_{1} \alpha+\mathbf{L}_{2} \alpha^{2} \\
\mathbf{L}_{00} & =\left[\begin{array}{cc}
\mathbf{L}_{000} & \mathbf{0} \\
\mathbf{0} & \mathbf{L}_{000}
\end{array}\right] .
\end{aligned}
$$

This second-order polynomial form for the inductance matrix can be utilised to compute the inverse of $\mathbf{L}$ by the Taylor expansion of a quadratic polynomial. This is applied to determine the capacitance matrix, which follows from:

$$
\mathbf{C}=\mu_{0} \varepsilon_{0} \varepsilon_{r} \mathbf{L}^{-1} \text {. }
$$

This can be approximated by Taylor expansions in the following way:

$$
\hat{\mathbf{C}}=\mu_{0} \varepsilon_{0} \varepsilon_{r}\left[\mathbf{I}_{4}-\mathbf{L}_{0}^{-1} \mathbf{L}_{1} \alpha-\left(\mathbf{L}_{0}^{-1} \mathbf{L}_{2}-\mathbf{L}_{0}^{-1} \mathbf{L}_{1} \mathbf{L}_{0}^{-1} \mathbf{L}_{1}\right) \alpha^{2}\right] \mathbf{L}_{0}^{-1} .
$$

It remains to determine the inverse of $\mathbf{L}_{0}$. Suppose that the wire pairs are closer to the reference ground plane than to each other $(h<d / 2 \sqrt{2})$, by which $x \ll 1$ and thus $b \ll 1$. In that case:

$$
\hat{\mathbf{L}}_{0}^{-1}=\left[\mathbf{I}_{4}-\mathbf{L}_{00}^{-1} \mathbf{L}_{01} b\right] \mathbf{L}_{00}^{-1} .
$$

The structure of inductance and capacitance matrices is utilised to obtain the final closed-form expression for the NEXT between two wire pairs close 
to ground. For presentation purpose, suppose that $\mathbf{Z}_{S}=\mathbf{Z}_{L}=\mathbf{Z}$ (subscript $S / L$ is dropped for $R_{d, m T}$ ). Combining (2.19) and (2.20), (2.26) and (2.28) then leads to closed-form expressions for inductive crosstalk:

$$
\gamma_{N E, \text { ind }} \approx \frac{-3 \mu_{0}}{2 \pi R_{d, c}}\left(\frac{R_{d, c}}{R_{c, c}}+2\right) a^{2} h^{2} \mathrm{j} \omega \ell d^{-4},
$$

and capacitive crosstalk:

$$
\gamma_{N E, c a p} \approx \frac{-24 \pi \varepsilon_{0} \varepsilon_{r} R_{d, v}}{\left(\frac{R_{d, v}}{R_{c, v}}+2\right)} \frac{a^{2} h^{2} \mathrm{j} \omega \ell d^{-4}}{\ln ^{2}\left(r^{2}\left(\frac{1}{4 h^{2}}+\frac{1}{a^{2}}\right)\right)} .
$$

The total NEXT follows from addition of the inductive and capacitive expressions:

$$
\gamma_{N E} \approx \gamma_{N E, i n d}+\gamma_{N E, \text { cap }} .
$$

The dependencies on all designable parameters are clearly shown by (2.30) and (2.31). The fourth-order dependency on wire pair separation is important. It implies that when the distance $d$ is doubled, the crosstalk decreases by 24 $\mathrm{dB}$. For most other wiring configurations this would be $12 \mathrm{~dB}$. This fourth-order dependency is validated by the results shown in Figure 2.5, which shows both MTL simulations and measurements of crosstalk between two wire pairs with a length of $1.9 \mathrm{~m}$. Crosstalk levels are shown for two separation distances, namely 10 and $20 \mathrm{~mm}$. Values for other parameters are: $a=2.5 \mathrm{~mm}, r=0.49$ $\mathrm{mm}, h=1.5 \mathrm{~mm}, \varepsilon_{r}=2, R_{d, c}=R_{d, v}=112.5 \Omega$ and $R_{c, c}=R_{c, v}=450 \Omega$ (equal load and source side terminations). Indeed, in both measurements and simulations the difference between results for the two separations is approximately $24 \mathrm{~dB}$. Moreover, measured crosstalk results agree with those from MTL simulations. Finally, Figure 2.6 shows similar results of MTL simulations and closedform expressions for the case where 4 extra wires, terminated with $50 \Omega$ to ground, are arbitrarily placed in between the two wire pairs. Three variations of the parameters $d$ and $h$ are shown, while other parameters are equal to the values above. Simulated results illustrate that extra conductors slightly affect the levels of crosstalk, but the parameter dependencies roughly remain the same. Situations with other terminations show that it is actually only the capacitive coupling between the wire pairs that is influenced by nearby conductors.

The measurements were performed between two wire pairs that were fixed to the given distances along the entire length of the cables by using spacers of Rohacell foam (see Figure 2.7 for pictures of the setup). Moreover, in the case that includes a ground plane foam spacers were used to keep the wires at fixed height above ground. The aluminium ground plane had a width of 1 $\mathrm{m}$, which is enough to avoid edge effects. Baluns were used to connect the balanced pairs to the unbalanced coaxes that are connected to the measurement equipment. The frequency dependent transmission of these baluns is calibrated from the measurement results. This is done by connecting two 


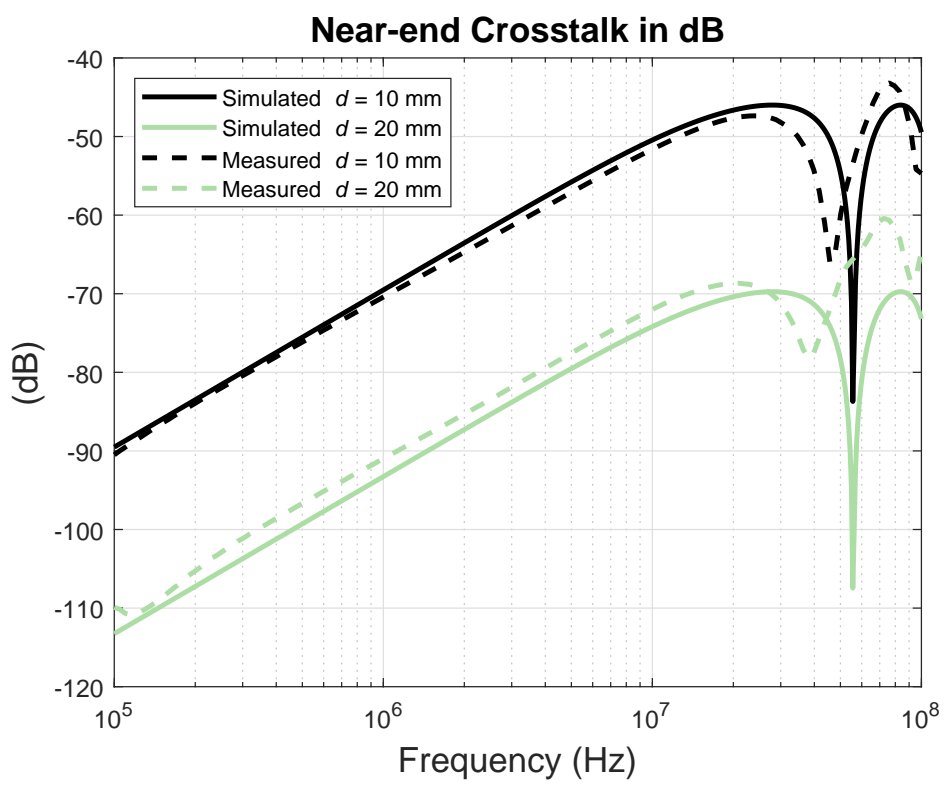

Figure 2.5: MTL simulations and measurements of NEXT for two separation distances in the wire configuration given in Figure 2.3a.

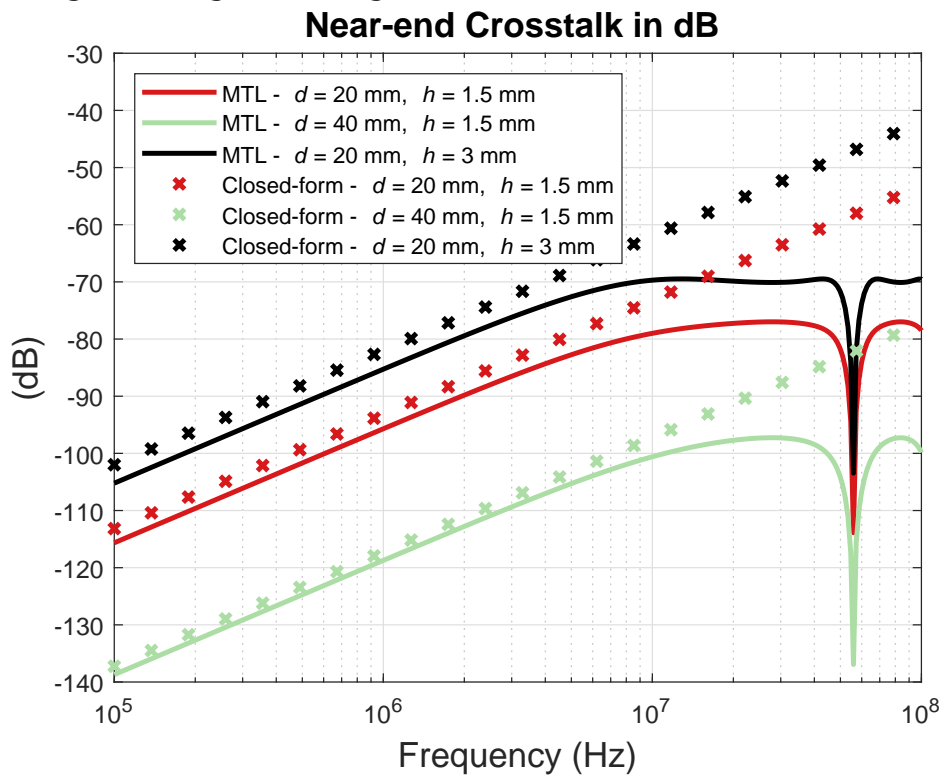

Figure 2.6: MTL simulations (solid lines) and closed-form expressions (2.32) (crosses) of NEXT for three different cases of the wire configuration given in Figure 2.3a, in which 4 terminated wires are included in between the wire pairs. 


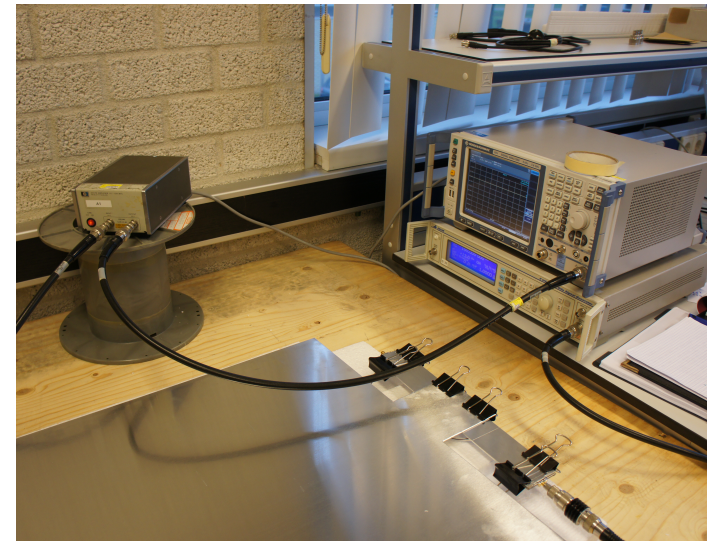

(a) Measurement equipment: Spectrum analyser, generator and preamplifier.

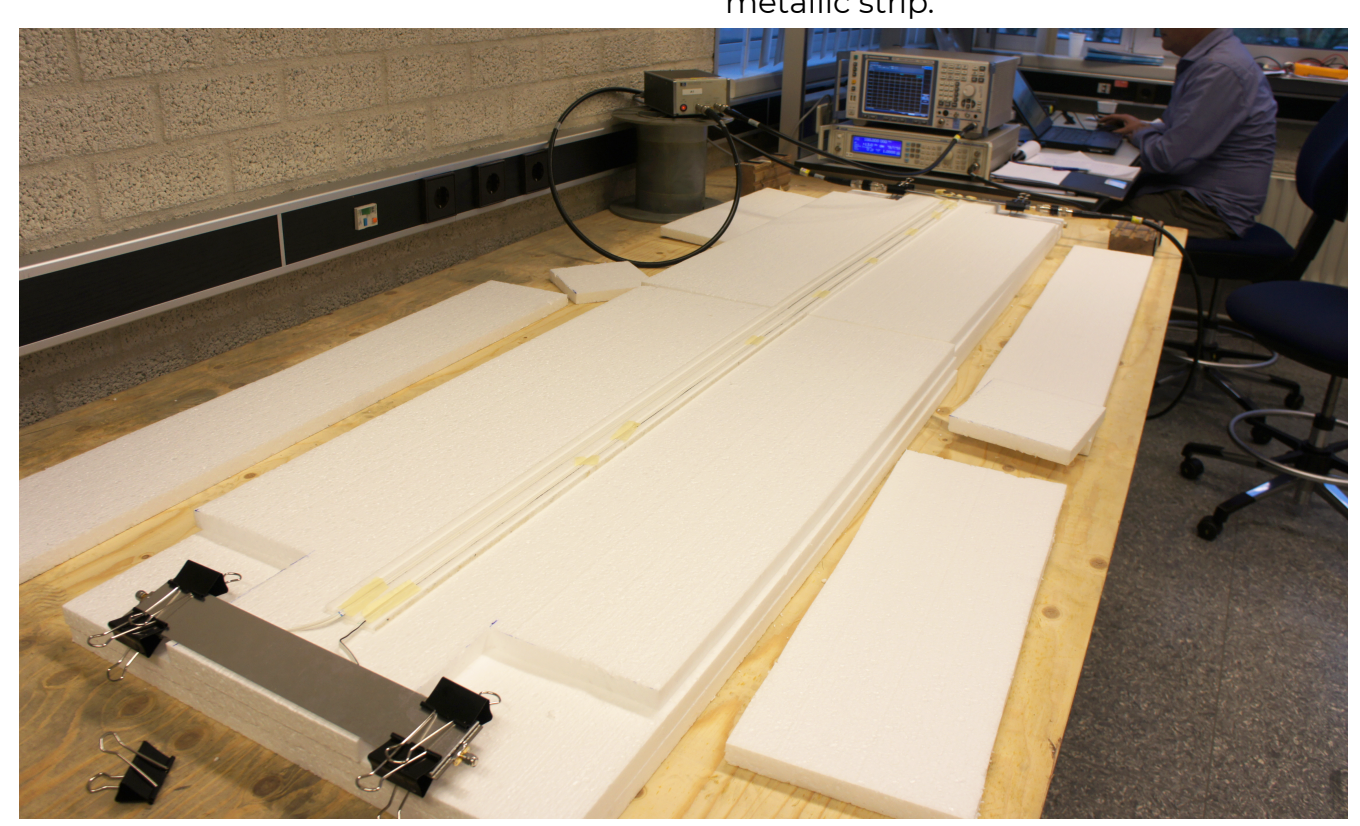

(c) Foam spacers are used to keep the wire pairs at fixed separation distances and heights above ground plane along the TL length.

Figure 2.7: Pictures of the crosstalk measurement setup.

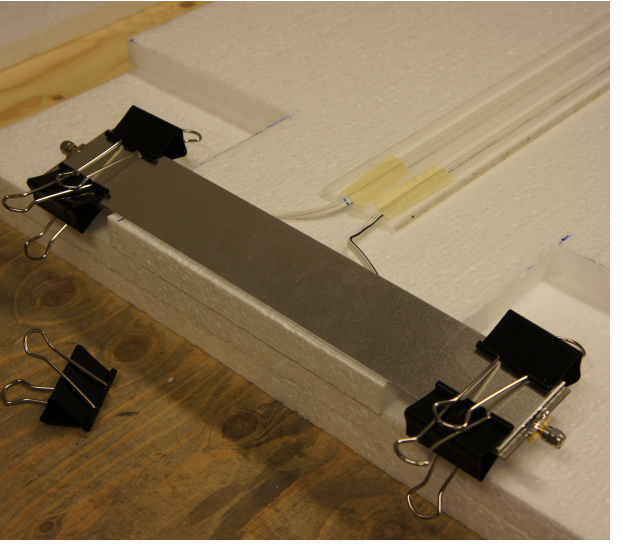
separated and soldered to balun boards that are attached to the backside of the metallic strip. 
baluns to each other with short pieces of wire and measuring the insertion loss. Obtained crosstalk measurement results are compensated for the obtained calibration measurement. The frequencies for which results are shown in this chapter are determined by the applicability of these baluns. This frequency band includes only little effect of ohmic and dielectric losses.

\subsubsection{Two wire pairs without nearby ground plane}

As a second example, consider the previous configuration without ground plane. An illustration of the cross-section of this case is shown in Figure 2.3b. All properties remain the same, including the termination impedances. However, in this case the fourth wire is taken as the reference. The other victim wire is numbered 1, and we finish by labelling the culprit wires 2 and 3. Impedance matrices $\mathbf{Z}_{S}$ and $\mathbf{Z}_{L}$ can be derived similar to the previous section (not shown here). Finally, a differential voltage source is included in the culprit pair, by which we can define $\mathbf{U}_{1}=(0,-1,1)^{T}$ and $\mathbf{U}_{2}=(1,0,0)^{T}$.

Near-end crosstalk Again (2.13) is used to compute the numerator and denominator of (2.3) for this wiring configuration:

$$
\begin{aligned}
\mathbf{U}_{1}^{T} \mathbf{v}_{0} \approx & \frac{-2 V_{S}\left(\bar{Y}_{C S}+2 \bar{K}_{C S}\right)}{\bar{Y}_{C L}+\bar{Y}_{C S}+2 \bar{K}_{C L}+2 \bar{K}_{C S}} \\
\mathbf{U}_{2}^{T} \mathbf{v}_{0} \approx & \frac{j \omega \ell V_{S}\left(\bar{Y}_{C L}+2 \bar{K}_{C L}\right)\left(\bar{Y}_{C S}+2 \bar{K}_{C S}\right)\left(\bar{Y}_{V L}+2 \bar{K}_{V L}\right)\left(l_{12}-l_{13}\right)}{2\left(\bar{Y}_{C L}+\bar{Y}_{C S}+2 \bar{K}_{C L}+2 \bar{K}_{C S}\right)\left(\bar{Y}_{V L}+\bar{Y}_{V S}+2 \bar{K}_{V L}+2 \bar{K}_{V S}\right)} \\
& +\frac{4 j \omega \ell V_{S}\left(\bar{Y}_{C S}+2 \bar{K}_{C S}\right)\left(2 c_{12}-2 c_{13}+c_{22}-c_{33}\right)}{2\left(\bar{Y}_{C L}+\bar{Y}_{C S}+2 \bar{K}_{C L}+2 \bar{K}_{C S}\right)\left(\bar{Y}_{V L}+\bar{Y}_{V S}+2 \bar{K}_{V L}+2 \bar{K}_{v S}\right)} .
\end{aligned}
$$

Here $\bar{Y}_{m T}=R_{d, m T}^{-1}$ and $\bar{\kappa}_{m T}=\left(4 R_{c, m T}\right)^{-1}$ are entries from the admittance matrices, in which again the subscript $m$ is either culprit or victim, and $T$ denotes either load or source side. Substituting this into (2.3) yields the following expressions for capacitive and inductive near-end crosstalk:

$$
\begin{aligned}
& \gamma_{N E, \text { cap }} \approx \mathrm{j} \omega \ell \frac{2 c_{12}-2 c_{13}+c_{22}-c_{33}}{4\left(\bar{Y}_{V L}+\bar{Y}_{V S}+2 \bar{K}_{V L}+2 \bar{K}_{V S}\right)} \\
& \gamma_{N E, \text { ind }} \approx \mathrm{j} \omega \ell \frac{\left(\bar{Y}_{C L}+2 \bar{K}_{C L}\right)\left(\bar{Y}_{V L}+2 \bar{K}_{V L}\right)\left(l_{13}-l_{12}\right)}{4\left(\bar{Y}_{V L}+\bar{Y}_{V S}+2 \bar{K}_{V L}+2 \bar{K}_{V S}\right)}
\end{aligned}
$$

Closed-form crosstalk expressions Paul's expressions for self and mutual inductances for cylindrical conductors in free space are given by:

$$
l_{i i}=\frac{\mu_{0}}{2 \pi} \ln \left(\frac{d_{i 0}^{2}}{r_{i} r_{0}}\right) \quad l_{i j}=\frac{\mu_{0}}{2 \pi} \ln \left(\frac{d_{i 0} d_{j 0}}{d_{i j} r_{0}}\right) .
$$


As in (2.21), $d_{i j}$ is the distance between conductors $i$ and $j$, in which for this case the subscript 0 refers to the reference conductor. Following these expressions, the inductance matrix for this case of wire pairs without nearby ground plane equals:

$$
\begin{aligned}
\mathbf{L} & =\mathbf{L}_{0}+\mathbf{L}_{\alpha} \\
\mathbf{L}_{0} & =\left[\begin{array}{lll}
2 & 1 & 1 \\
1 & 2 & 1 \\
1 & 1 & 2
\end{array}\right] p+\left[\begin{array}{ccc}
0 & 0 & 0 \\
0 & -1 & -1 \\
0 & -1 & -1
\end{array}\right] q \\
\mathbf{L}_{\alpha} & =\left[\begin{array}{ccc}
0 & -\beta \ln (1-\alpha) & \beta \ln (1+\alpha) \\
-\beta \ln (1-\alpha) & 0 & \beta \ln (1+\alpha) \\
\beta \ln (1+\alpha) & \beta \ln (1+\alpha) & 2 \beta \ln (1+\alpha)
\end{array}\right],
\end{aligned}
$$

where $p=\beta \ln (a / r)$ and $q(d)=2 \beta \ln (a / d)$. Taylor expansion of the inductance matrix with respect to the small parameter $\alpha$ leads to:

$$
\begin{aligned}
\hat{\mathbf{L}}_{1} & =\mathbf{L}_{1} \alpha+\mathbf{L}_{2} \alpha^{2} \\
& =\left[\begin{array}{lll}
0 & 1 & 1 \\
1 & 0 & 1 \\
1 & 1 & 2
\end{array}\right] \beta \alpha+\left[\begin{array}{ccc}
0 & 1 & -1 \\
1 & 0 & -1 \\
-1 & -1 & -2
\end{array}\right] \frac{\beta}{2} \alpha^{2} .
\end{aligned}
$$

The capacitance matrix is approximated as before by (2.28). The inverse of $\mathbf{L}_{0}$ has to be calculated explicitly in terms of $p$ and $q$, since it cannot be expressed in terms of a small parameter:

$$
\mathbf{L}_{0}^{-1}=\frac{1}{4(p-q)}\left[\begin{array}{ccc}
3-2 g & -1 & -1 \\
-1 & 3-2 g & -1+2 g \\
-1 & -1+2 g & 3-2 g
\end{array}\right]
$$

where $g=q / p$. The above approximations for the capacitance and inductance matrices can be used to obtain a closed-form expression for NEXT for wire pairs without nearby ground plane. Again we assume for simplification of the formulas that the terminations on both sides of the transmission lines are equal (and drop the source/load subscripts on terminations). Then (2.34) with low-frequency approximations of inductance and capacitance yields the desired closed-form expressions for NEXT:

$$
\begin{aligned}
& \gamma_{N E, \text { cap }} \approx \frac{-\pi \varepsilon_{0} \varepsilon_{r}\left(1+\ln ^{-1}\left(\frac{d^{2}}{r a}\right)\right)}{4 \ln ^{2}\left(\frac{a}{r}\right)\left(\frac{1}{R_{d, v}}+\frac{1}{2 R_{c, v}}\right)} a^{2} \mathrm{j} \omega \ell d^{-2} \\
& \gamma_{N E, i n d} \approx \frac{-\mu_{0}}{4 \pi}\left(\frac{1}{R_{d, c}}+\frac{1}{2 R_{c, c}}\right) a^{2} \mathrm{j} \omega \ell d^{-2} .
\end{aligned}
$$

Again, the sum of inductive and capacitive coupling gives the total NEXT. The relation between crosstalk and all designable parameters is shown by these 
equations. The dependency on the separation distance is quadratic, as opposed to the fourth-order behaviour of the previous section. This is confirmed by the comparison of two measured and two simulated results for separation distances of $10 \mathrm{~mm}$ and $20 \mathrm{~mm}$, as shown in Figure 2.8. All simulation parameters are equal to the previous case. The difference in crosstalk between these traces is roughly $12 \mathrm{~dB}$. Moreover, there is again a good match between measurements and MTL simulations.

In addition to separation dependency, Figure 2.9 shows MTL simulations and measurements of near-end crosstalk that focus on the height above the ground plane. Results are given for varying heights of the pairs above a ground plane, as well as for the situation without a ground plane. Measurements and simulations are in good agreement. Moreover, the $12 \mathrm{~dB}$ difference between the simulated results for $h$ is $1.5 \mathrm{~mm}$ and $3 \mathrm{~mm}$ confirms the quadratic dependency found from (2.32). Finally, both measured and simulated results in Figure 2.9 indicate that when moving wire pairs away from the ground plane the crosstalk levels approach those for wire pairs without nearby ground plane.

\subsubsection{Parameter dependencies}

Closed-form expressions for low-frequency crosstalk are useful to extract dependencies of crosstalk on designable parameters. Table 2.1 gives an overview of all leading-order dependencies for both inductive and capacitive crosstalk. Each entry of the table is given by the order with which the corresponding parameter appears in the closed-form expression, with a plus or minus sign showing if crosstalk increases or decreases with an increase of the parameter value. The sensitivities have been derived from (2.32) and (2.39), which were obtained under the assumption that $a \ll d$, as well as $h \ll d$ in case a ground plane is involved. Specifically, when $2 a \leq d$ and $d \geq 2 h \sqrt{2}$ hold, nearly all dependencies given in Table 2.1 hold. For inductive coupling these sensitivities are simply read from the exponents in (2.30) and (2.34). For capacitive coupling logarithms complicate the dependencies on $a, h$ and $r$. For the case with ground plane, the logarithm in the denominator of (2.31) can be simpli-

Table 2.1: Near-end crosstalk between wire pairs: sensitivity to model parameters*

\begin{tabular}{llcccccccc}
\hline \hline Ground & & & & & & & & & \\
plane & Type & $d$ & $a$ & $h$ & $r$ & $Z$ & $f$ & $\ell$ & $\varepsilon_{r}$ \\
\hline Yes & Inductive & -4 & +2 & +2 & 0 & -1 & +1 & +1 & 0 \\
Yes & Capacitive & -4 & $+7^{*}$ & $+2^{*}$ & $>+1$ & +1 & +1 & +1 & +1 \\
No & Inductive & -2 & +2 & - & 0 & -1 & +1 & +1 & 0 \\
No & Capacitive & -2 & +1 & - & $>+1$ & +1 & +1 & +1 & +1 \\
\hline
\end{tabular}

* In general, all orders of dependency hold for $2 a \leq d$ and $d \geq h 2 \sqrt{2}$. The only extra assumption is needed for ${ }^{*}$, in which the complex ratio of (2.31) is simplified assuming $a \leq 2 h$. 


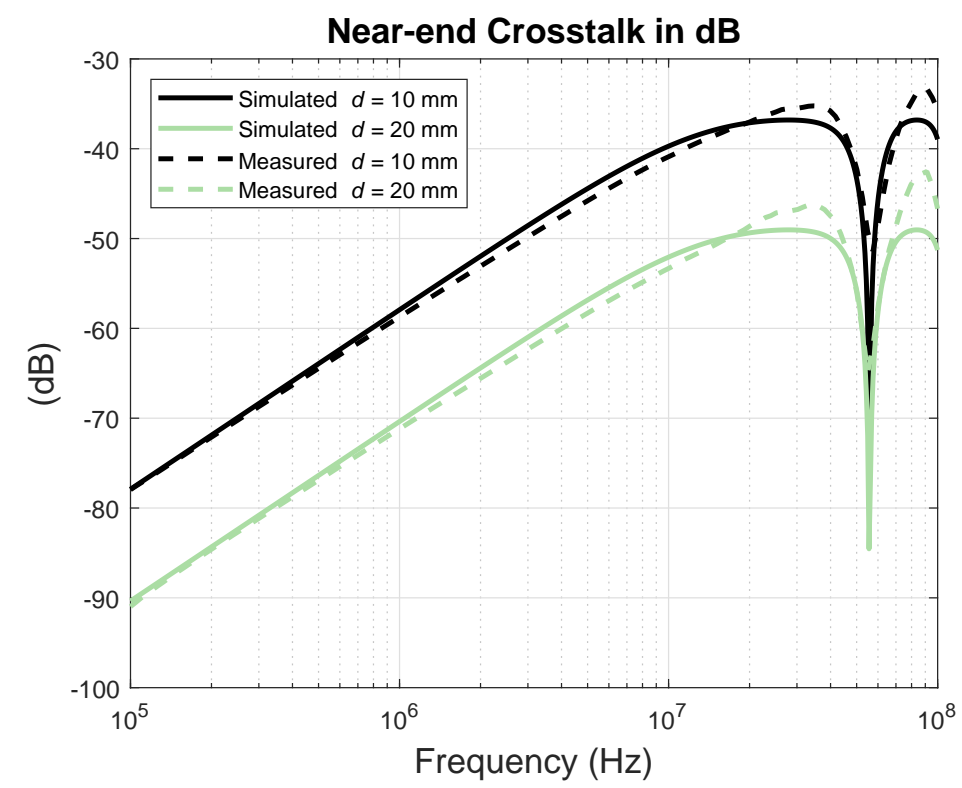

Figure 2.8: MTL simulations and measurements of NEXT for two separation distances in the wire configuration given in Figure 2.3b.

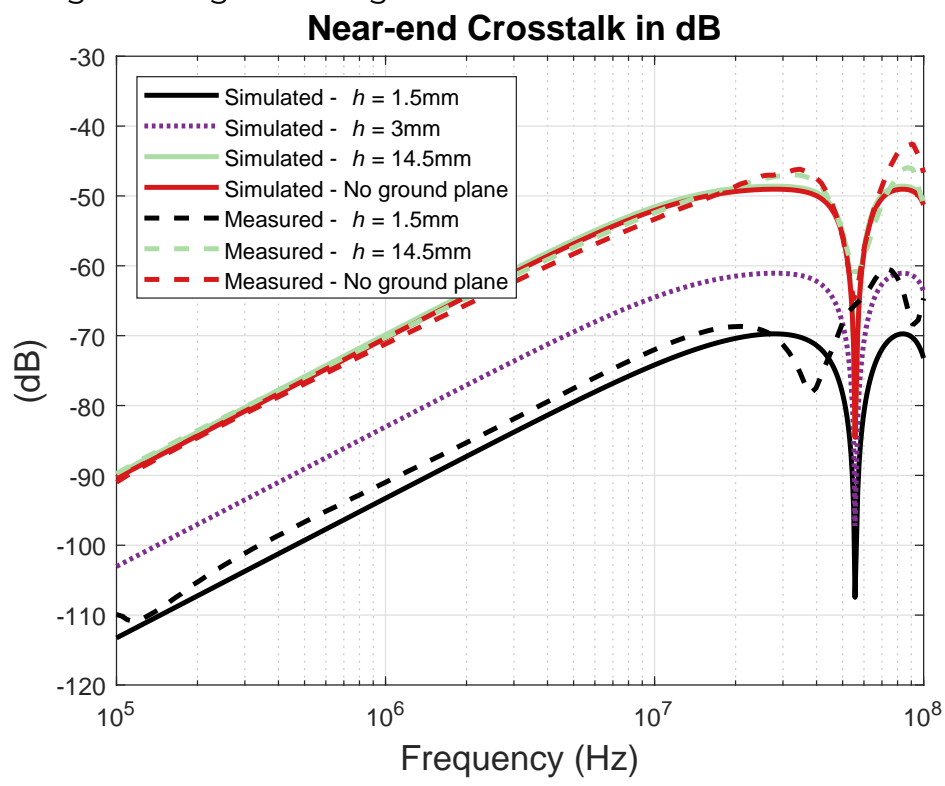

Figure 2.9: NEXT simulations (solid lines) and measurements (dashed lines) of NEXT for three values of the height above a ground plane in the wire configuration given in Figure 2.3a. For $h=3 \mathrm{~mm}$ (purple, dotted line) only MTL simulation data is available. One result for the situation without ground plane (Figure 2.3b) is given (red). 
fied when $a \leq 2 h$, which is a reasonable assumption when the wires of a pair are separated only by some insulation. In that case it can be derived that the dependency on height is quadratic. The dependency on intra-pair separation is then such that the increase in crosstalk is roughly 5-8 dB when $a$ is doubled, hence the dependency stated by Table 2.1 is roughly first-order. Similar intra-pair separation dependency holds for the case without ground plane. Note that when any of the given assumptions is violated, the order of dependency for certain parameters might change. However, the trend of crosstalk behaviour with respect to that parameter will usually remain equal.

Clearly, the parameter with highest sensitivity is the separation distance between two pairs close to a ground plane. Wire radius does not affect the inductive crosstalk at all, while the dependency of capacitive crosstalk on wire radius still involves a complex logarithm. Therefore its sensitivity on $r$ is such that crosstalk increases roughly $10 \mathrm{~dB}$ when $r$ is doubled, implying slightly more than linear behaviour, hence the $>+1$ in the table.

The impedance parameter $\boldsymbol{Z}$ given in Table 2.1 refers to the differential mode impedance between either the victim or culprit pair for capacitive or inductive crosstalk, respectively. For both types of crosstalk, the dependency on termination impedances is of order one, but inductive crosstalk is always inversely proportional to culprit terminations, while capacitive crosstalk is linearly dependent on victim impedance.

\subsection{Non-uniform transmission lines}

Apart from its use in the derivation of closed-form expressions, the set of equations given in (2.10) can also be applied to non-uniform transmission lines. For instance, to compute crosstalk in a bundle of twisted wire pairs the most common solution is to use a sufficient amount of Uniform Cascased Sections (UCS) and to multiply the chain parameter matrices for each section [6], [7]. Using the exact form of the chain parameter matrices involves performing eigen decompositions for each uniform section. However, each section is usually small in terms of wavelengths. Therefore, when quick computations are desired above the inclusion of high-frequency losses, the approximate low-frequency form (2.10) can be used for each segment.

Figure 2.10 shows simulation results for a bundle of seven $1 \mathrm{~m}$ long twisted pairs that are separated $3.4 \mathrm{~mm}$ from each other and are enclosed by a bundle shield. Near-end crosstalk between the centre pair and one of the surrounding pairs is considered. More details of this bundle are given in Chapter 5 and in [35]. The results for full MTL simulation including finite wire conductivity ( $\sigma=$ $6 e 7 \mathrm{~S} / \mathrm{m}$ ) are compared to those of a lossless MTL simulation and a simulation in which (2.10) is used for the chain parameter matrices of each uniform section. This approximate result exactly matches that of lossless MTL simulation, while the difference with respect to the full MTL simulation obtains a maximum of only $0.86 \mathrm{~dB}$ in the resonance area. The computation time of the full 
MTL simulation, on a single core of a simple laptop with Intel i5 processor and $8 \mathrm{~GB}$ of RAM, is more than 20 times higher than the time needed for the approximate solution, while this is 10 times when compared to the lossless MTL simulation. Therefore, the solution that utilises (2.10) forms a better candidate for cable optimisations, sensitivity analyses and early risk assessments than the usual MTL simulations. This will be applied in sensitivity analyses of more complex cable bundles in Chapter 5 .

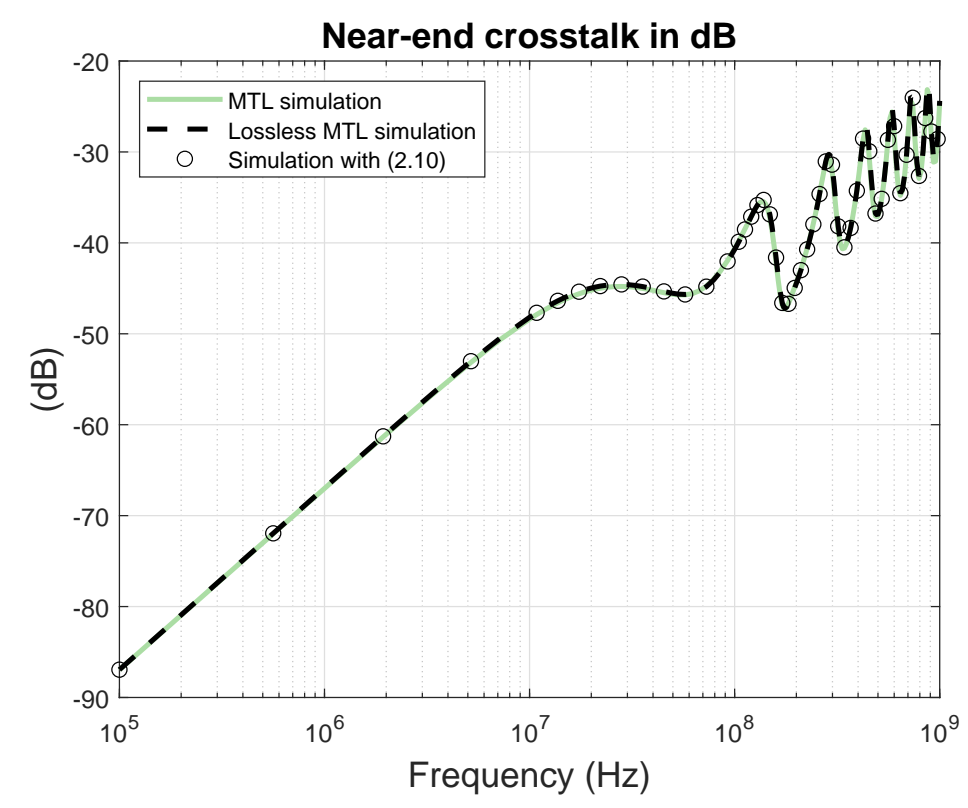

Figure 2.10: Simulated NEXT for a bundle of 7 twisted pairs. Results are compared for full MTL simulation, lossless MTL simulation and the low-frequency approximation in (2.10) applied to each uniform segment.

\subsection{Summary}

Efficient crosstalk design rules are required to optimise cable bundles and to yield early routing of low-risk signals as well as early risk identification for highrisk signals. This chapter presented a mathematical methodology to analyse crosstalk in multi-conductor transmission lines. With the proposed lowfrequency technique closed-form expressions that directly relate crosstalk to designable parameters which are valid for electrically short cables are derived.

For two cable configurations involving wire pairs with and without a nearby perfectly conducting ground plane, the low-frequency analysis has resulted in expressions for near-end crosstalk that clearly show the differences between all designable parameter sensitivities. For instance, in the configuration with 
ground plane the crosstalk decreases up to $24 \mathrm{~dB}$ when the inter-pair separation distance is doubled. However, when the height of the wire pairs above the ground plane becomes large compared to the inter-pair separation distance, the crosstalk behaviour approaches that of wire pairs without nearby ground plane. In that case, crosstalk increases with a more familiar $12 \mathrm{~dB}$ when doubling the separation distance.

Using the efficient low-frequency approximations of the chain parameter matrices leads to accurate lossless results when analysing more complex nonuniform MTLs with UCS. However, the computation time becomes more than 20 times smaller when compared to the usual MTL simulations. Therefore, in this case, the low-frequency approximations can be well applied to perform quick analysis of cable bundles that involve twisted pairs, meandering of cables and other complex non-linear phenomena. 


\section{3 | Shielded cables}

Crosstalk in situations where a possible common return conductor is lossless can be subdivided into coupling due to magnetic and electric fields, i.e. inductive and capacitive crosstalk, respectively. Depending on the impedances of connected systems the magnitudes of inductive and capacitive coupling change. In general, crosstalk is a combination of electric and magnetic field effects. However, in specific cases one of the two might be dominant. In Chapter 2 closed-form expressions were derived for crosstalk between single wires and wire pairs that explicitly reveal also the dependencies on termination impedances. For instance, it shows that for high victim termination impedances the capacitive crosstalk increases, making it likely that electric coupling effects dominate crosstalk behaviour. There are several solutions to minimise crosstalk, of which shielding is an effective method to reduce both electric and magnetic field coupling.

To include shields in crosstalk models the theory of Paul [5] can be extended. An important aspect in solving the MTL equations is the determination of the p.u.l. parameters for the cross-section of shielded wires. Paul considers a method to include a single shield around a single wire into the p.u.l. parameters and the MTL equations in several papers [36,37]. In this chapter we extend this method for other shielding configurations that involve two single shields, as well as a double shield. Moreover, we derive a closed-form expression for CM crosstalk in three different configurations where two wires are situated parallel to an infinite, perfectly conducting ground plane and carry either one or two shields. Such trends in the frequency domain for a single wire versus a single shielded wire were also analysed by Paul. However, in this chapter for multiple shielding configurations the derivations lead to expressions for crosstalk levels that clearly relate to all designable parameters. These expressions show interesting behaviour of the shields with respect to frequency. For double shielded wires five different frequency regions are observed. The presence of one shield gives rise to a region where crosstalk remains constant with frequency. The addition of a second shield could cause another such interval. Analysis of the transition frequencies and the crosstalk levels leads to generic predictions of crosstalk in the frequency domain. In a very practical way this can be advantageous for the design of cabling. For instance cable manufacturers can analyse effects of several parameters on crosstalk levels. 
The generic prediction could be used to optimise shielding parameters with the aim to achieve desired crosstalk levels in certain frequency domains.

This chapter presents the derivation of such closed-form expressions for NEXT for double shielded wires, while afterwards the generic predictions are given for three different shielding configurations:

- Single wire versus a single shielded wire above a perfectly conducting ground plane.

- Two single shielded wires above a perfectly conducting ground plane.

- Single wire versus a double shielded wire above a perfectly conducting ground plane.

For wires that carry a shield, the electromagnetic interactions between in and outside are modelled by transfer impedance. If the wire carries a double shield this is modelled by two separate transfer impedances. In general, both transfer impedance and transfer admittance govern the relations between magnetic and electric fields in and outside the shields, respectively. However, the effects of transfer admittance are usually negligibly small, and therefore out of scope for this thesis. More details on the transfer impedance are discussed in Chapter 5. This chapter focuses on the derivation of crosstalk expressions when a certain transfer impedance is given.

In Section 3.1 the properties of the double shield are included into the p.u.l. parameters for inductance, capacitance and resistance. For the case of a double shielded wire and an unshielded wire above a ground plane the p.u.l. parameters are described by four-dimensional matrices. By substituting these matrices into the MTL equations, in Section 3.2 closed-form expressions can be obtained for the voltages and crosstalk levels. To fully derive the effects of the shields we first solve the shield currents from the corresponding MTL equations. Next, we substitute these obtained expressions into the remaining two equations. This results into an augmented two-dimensional system for two wires above a ground plane. Analytical expressions for several shielding configurations can then be obtained by solving this augmented system by use of Taylor expansions in the frequency domain, similar to the previous chapter and [23]. This procedure leads to results corresponding with [36] when applied to the case where the double shield is replaced by a single shield.

The knowledge about effects of shielding is increased by analysing generic predictions of crosstalk in the frequency domain. In Section 3.3 such generic predictions for three different shielded situations versus one case with two unshielded wires are compared. It illustrates the practical use of analysing crosstalk levels with the introduced approach. The final section of this chapter gives a summary of the results. 


\subsection{Transmission line characteristics}

Consider a situation with two wires parallel to an infinite, perfectly conducting ground plane, where one of the wires is surrounded by a double shield. The cross-section is illustrated in Figure 3.1 and the terminations in Figure 3.2. In the following we describe the modelling of this wiring configuration.

\subsubsection{Per-Unit-Length parameters}

The electromagnetic analysis of such a transmission line requires determination of the per-unit-length parameters. In this chapter the medium surrounding all cable configurations is free space and therefore homogeneous and lossless. Modelling of shields in the p.u.l. parameters is based upon papers by Paul $[36,37]$, in which p.u.l. matrices were derived for a situation with one single shield. Here we extend these matrices to a double shielded configuration (see Figure 3.1, which also gives numbering of conductors). Afterwards, in the next section we derive crosstalk expressions for this configuration.

Inductance The derivation of the p.u.l. inductance matrix is obtained by domain decomposition. To this extent, all currents and voltages are defined with respect to their local reference (see Figure 3.3), e.g. the return current of conductor two flows in the inner shield and the return current of that inner shield flows in the outer shield. A local inductance matrix $\mathbf{L}^{\prime}$ can then be defined as follows [37,38]:

$$
\mathbf{L}^{\prime}=b\left[\begin{array}{cccc}
\ln \left(\frac{2 h}{r}\right) & 0 & 0 & \frac{1}{2} \ln (1+x) \\
0 & \ln \left(\frac{2 h}{r}\right) & 0 & 0 \\
0 & 0 & \ln \left(\frac{2 h}{r_{s, 1}}\right) & 0 \\
\frac{1}{2} \ln (1+x) & 0 & 0 & \ln \left(\frac{2 h}{r_{s, 2}}\right)
\end{array}\right]
$$

Here $x=4 h^{2} / d^{2}$ and $b=\mu_{0} / 2 \pi$. The geometrical parameters are defined in Figure 3.1.

The domain decomposition matrices $\mathbf{T}_{I}$ and $\mathbf{T}_{V}$ that relate the global or physical (unprimed) voltages and currents to the local or modal (primed) ones in Figure 3.3, are found by writing:

$$
\begin{aligned}
& I_{1}^{\prime}=I_{1}, \quad I_{2}^{\prime}=I_{2}, \quad I_{3}^{\prime}=I_{2}+I_{3}, \quad I_{4}^{\prime}=I_{2}+I_{3}+I_{4} \\
& V_{1}^{\prime}=V_{1}, \quad V_{2}^{\prime}=V_{2}-V_{3}, \quad V_{3}^{\prime}=V_{3}-V_{4}, \quad V_{4}^{\prime}=V_{4} \text {, }
\end{aligned}
$$

This results in:

$$
\mathbf{T}_{I}=\left[\begin{array}{llll}
1 & 0 & 0 & 0 \\
0 & 1 & 0 & 0 \\
0 & 1 & 1 & 0 \\
0 & 1 & 1 & 1
\end{array}\right] \quad \mathbf{T}_{V}=\left[\begin{array}{rrrr}
1 & 0 & 0 & 0 \\
0 & 1 & -1 & 0 \\
0 & 0 & 1 & -1 \\
0 & 0 & 0 & 1
\end{array}\right]
$$




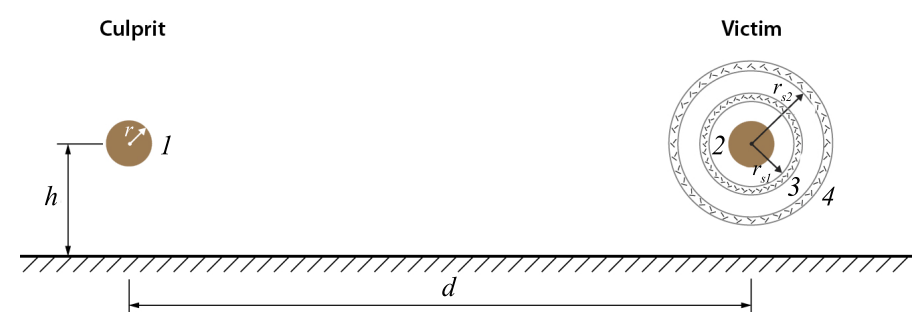

Figure 3.1: Unshielded versus double shielded wire above an infinite, perfectly conducting ground plane. Both wires have equal radius $r$ and height $h$.

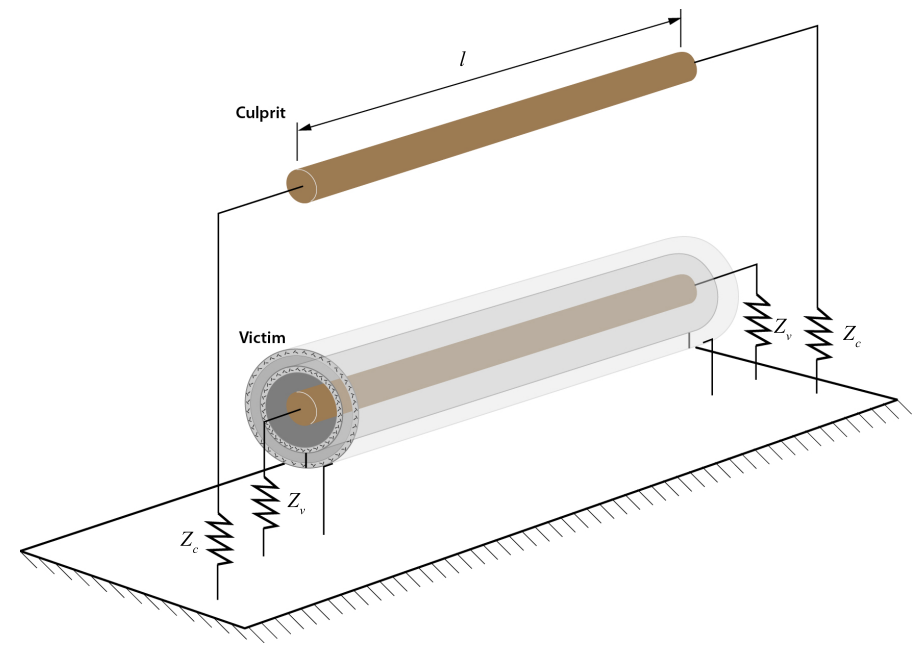

Figure 3.2: Finishing of the configuration with one unshielded wire and one double shielded wire.

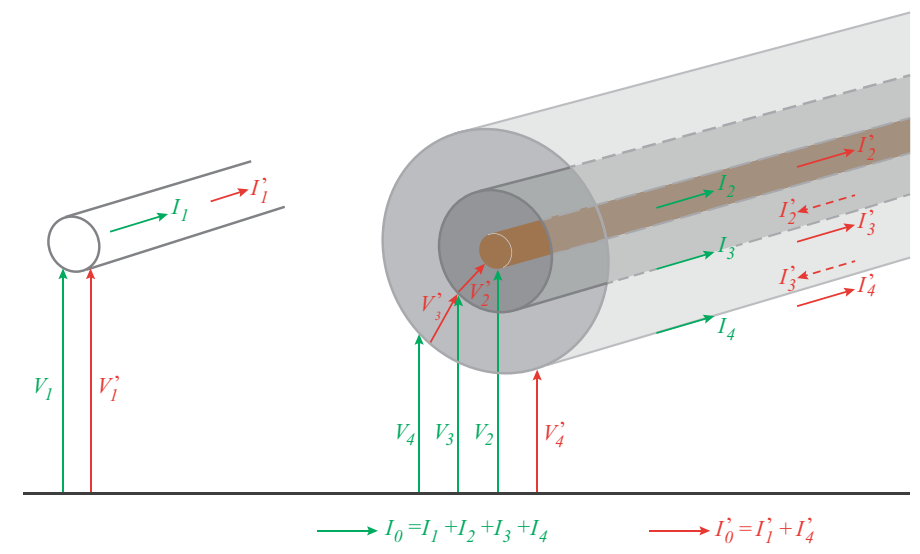

Figure 3.3: Illustration of local or modal (primed variables given in red) and global or physical (unprimed variables given in green) currents and voltages for the case of a single wire and a double shielded wire above ground. 
Then the global inductance matrix equals:

$$
\mathbf{L}=\mathbf{T}_{V}^{-1} \mathbf{L}_{I}=b\left[\begin{array}{cccc}
\ln \left(\frac{2 h}{r}\right) & \frac{1}{2} \ln (1+x) & \frac{1}{2} \ln (1+x) & \frac{1}{2} \ln (1+x) \\
\frac{1}{2} \ln (1+x) & \ln \left(\frac{2 h}{r}\right) & \ln \left(\frac{2 h}{r_{s, 1}}\right) & \ln \left(\frac{2 h}{r_{s, 2}}\right) \\
\frac{1}{2} \ln (1+x) & \ln \left(\frac{2 h}{r_{s, 1}}\right) & \ln \left(\frac{2 h}{r_{s, 1}}\right) & \ln \left(\frac{2 h}{r_{s, 2}}\right) \\
\frac{1}{2} \ln (1+x) & \ln \left(\frac{2 h}{r_{s, 2}}\right) & \ln \left(\frac{2 h}{r_{s, 2}}\right) & \ln \left(\frac{2 h}{r_{s, 2}}\right)
\end{array}\right] .
$$

Capacitance Both shields are directly connected to the ground. Therefore the capacitances between the culprit wire and the inner conductors (referred to by number 2 and 3 in Figure 3.4) are neglected. In Figure 3.4 all non-zero capacitances are shown. Together these are contained in the following capacitance matrix:

$$
\mathbf{C}=\left[\begin{array}{cccc}
c_{11}+c_{14} & 0 & 0 & -c_{14} \\
0 & c_{23} & -c_{23} & 0 \\
0 & -c_{23} & c_{23}+c_{34} & -c_{34} \\
-c_{14} & 0 & -c_{34} & c_{44}+c_{14}+c_{34}
\end{array}\right]
$$

Here it is assumed that (2.27) holds for the culprit wire versus outer shield, resulting in:

$$
\left[\begin{array}{cc}
c_{11}+c_{14} & -c_{14} \\
-c_{14} & c_{44}+c_{14}
\end{array}\right]=\mu_{0} \varepsilon_{0}\left[\begin{array}{ll}
l_{11} & l_{14} \\
l_{14} & l_{44}
\end{array}\right]^{-1}
$$

For the capacitances between the victim wire and inner shield, and inner and outer shield, respectively we have:

$$
c_{23}=\frac{2 \pi \varepsilon_{0}}{\ln \left(r_{s, 1} / r\right)}, \quad c_{34}=\frac{2 \pi \varepsilon_{0}}{\ln \left(r_{s, 2} / r_{s, 1}\right)} .
$$

Transfer impedance The coupling of the electromagnetic fields through the shields is governed by transfer impedance. In our case, this property is included in the p.u.l. impedance matrix, which reads:

$$
\hat{\mathbf{z}}=\operatorname{diag}\left(\begin{array}{llll}
0 & 0 & Z_{T, 1} & Z_{T, 2}
\end{array}\right)+j \omega \mathbf{L} \text {. }
$$

The transfer impedance of the two shields are given by $Z_{T, k}$ where $k$ is the number of the shield. In literature accurate models for this quantity can be found [39], though here for illustration purposes we use a simplified description:

$$
Z_{T, k}=R_{T, k}+j \omega l_{t}, \quad \text { with } \quad l_{t}=2 / \pi n F / m .
$$

The transfer impedance of shield $k$ comprises the constant Direct Current (DC) resistance part of the transfer impedance $R_{T, k}$ and its inductive part $l_{t}$. 


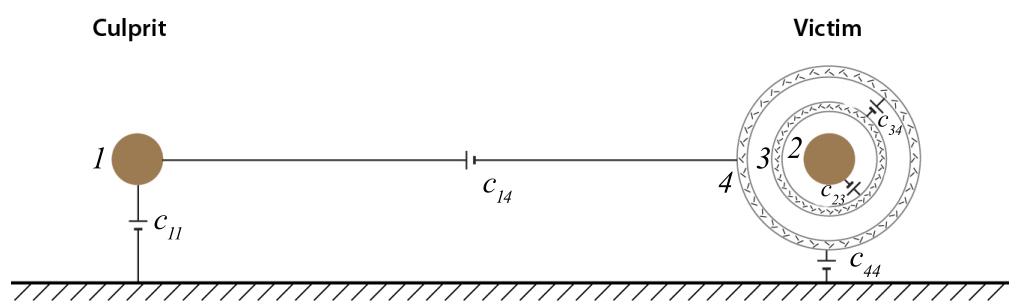

Figure 3.4: Capacitances for the configuration of one unshielded wire and one double shielded wire.

\subsubsection{Impedance matrices}

Besides the p.u.l. parameters also termination networks determine the behaviour of transmission lines. In Figure 3.2 it is seen that for this wiring situation the terminations of the TLs are straightforward and equal at both sides. This results in a four by four impedance matrix $\mathbf{Z}=\operatorname{diag}\left(Z_{c} Z_{v} 00\right)$ in which the only non-zero entries are given by $Z_{c}$ and $Z_{V}$, representing the culprit and victim termination impedance, respectively.

\subsection{Near-end crosstalk}

Like in Chapter 2 we define $\mathbf{V}_{0}$ as the vector of voltage differences between each conductor and the ground plane. The vector $\mathbf{I}_{0}$ contains the corresponding currents flowing in each conductor. Each pair of conductors forms a transmission line and between each pair of transmission lines electromagnetic coupling might occur which causes crosstalk. The common-mode near-end crosstalk between two transmission lines is computed as in equation (2.3) with in this case, $\mathbf{U}_{1}=\left(\begin{array}{llll}1 & 0 & 0 & 0\end{array}\right)^{T}$ and $\mathbf{U}_{2}=\left(\begin{array}{llll}0 & 1 & 0 & 0\end{array}\right)^{T}$.

\subsubsection{One double shielded versus one unshielded wire}

Starting point for the derivation of explicit analytic crosstalk expressions is the solution of the vector of voltages, which is required for the computation of crosstalk following equation (2.3). As in Chapter 2, these voltages can be obtained by solving equations (2.5) and (2.6), in which for this case $\mathbf{Z}_{S}=\mathbf{Z}_{L}=\mathbf{Z}$. The closed-form expressions follow by making use of the first-order approximation of the chain matrices as given by equation (2.10). A voltage source (see Figure 3.2) is present only at one side of the culprit transmission line, implying $\mathbf{V}_{L}=\mathbf{0}$ and $\mathbf{V}_{S}=V_{S} \mathbf{U}_{1}$.

The behaviour of the shields governed by the transfer impedance is contained in the chain matrices via the impedance matrix. To preserve this behaviour in the low-frequency solution of the matrix equation (2.6) we first solve the two equations for the shield currents. If subsequently these currents are 
substituted along with the chain matrices into the other two equations of (2.6), we find a two-dimensional matrix equation given by:

$$
[2 \mathbf{Z}+j \omega \ell[\mathbf{L}+\mathbf{Z C Z}-\mathbf{S}]] \mathbf{I}_{0}=\left[\mathbf{I}_{2}+j \omega \ell \mathbf{Z C}\right] \mathbf{V}_{S}
$$

In order to drop two dimensions in the p.u.l. and impedance matrices that were defined in the previous section, all row or column entries concerning the shields are deleted. The matrix $\mathbf{S}$ is given by:

$$
\mathbf{S}=j \omega \ell g\left[\begin{array}{ll}
S_{11} & S_{12} \\
S_{21} & S_{22}
\end{array}\right]
$$

in which:

$$
\begin{aligned}
& S_{11}=l_{12}^{2} Z_{T, 2} \ell+l_{12}^{2}\left(Z_{T, 1} \ell+j \omega l l_{33}\right)-l_{12}^{2} l_{44} j \omega l \\
& S_{12}=S_{21}=l_{12} l_{33}\left(Z_{T, 2} \ell+j \omega l l_{44}\right)+l_{12} l_{44} Z_{T, 1} \ell-l_{12} l_{44}^{2} j \omega l \\
& S_{22}=l_{33}^{2}\left(Z_{T, 2} \ell+j \omega \ell l_{44}\right)+l_{44}^{2} Z_{T, 1} \ell-l_{33} l_{44}^{2} j \omega l,
\end{aligned}
$$

and

$$
g=\left[\left(Z_{T, 1} \ell+j \omega \ell l_{33}\right)\left(Z_{T, 2} \ell+j \omega \ell l_{44}\right)-\left(j \omega \ell l_{44}\right)^{2}\right]^{-1} .
$$

By using a low-frequency Taylor approximation valid for electrically short lines to (3.10) together with equation (2.5) we obtain the voltage vector:

$$
\mathbf{V}_{0} \approx \frac{V_{S}}{2}\left[\mathbf{I}_{2}+\frac{1}{2} \mathrm{j} \omega \ell\left(\mathbf{L Z}^{-1}-\mathbf{Z C}-\mathbf{S Z}^{-1}\right)\right] \mathbf{U}_{1}
$$

Finally we compute the numerator and denominator of (2.3):

$$
\begin{aligned}
& \mathbf{U}_{1}^{T} \mathbf{v}_{0}=\frac{V_{S}}{2}+O(\omega \ell) \\
& \mathbf{U}_{2}^{T} \mathbf{v}_{0}=\frac{V_{S}}{4} Z_{c}^{-1} j \omega \ell\left(l_{21}-S_{21}\right)+O\left(\omega^{2} \ell^{2}\right)
\end{aligned}
$$

which leads to the following closed-form expression for the near-end crosstalk between two wires, of which one carries a double shield:

$$
\gamma_{N E} \approx \frac{j \omega \ell b \ln \left(1+4 h^{2} / d^{2}\right) Z_{T, 1} Z_{T, 2}}{4 Z_{C}\left[Z_{T, 1}^{*} Z_{T, 2}^{*}-\left(j \omega b \ln \left(2 h / r_{s, 2}\right)\right)\right]^{2}},
$$

in which the extended impedance $Z_{T, k}^{*}$ is given by $Z_{T, k}^{*}=Z_{T, k}+\mathrm{j} \omega b \ln \left(2 h / r_{s, k}\right)$ for $k \in\{1,2\}$. Inspection of (3.15) and (3.16) reveals that the near-end crosstalk is governed by inductive coupling only. Capacitive crosstalk vanishes because our model assumes that the shields are grounded at both sides.

Figure 3.5 shows that there is a good comparison between MTL simulations and the expression given by (3.16) for frequencies up to roughly $10 \mathrm{MHz}$. For simulations we used the following values for the model parameters: $h=1.67$ $\mathrm{mm}, r=0.16 \mathrm{~mm}, d=10 \mathrm{~mm}, \ell=2 \mathrm{~m}, r_{s, 1}=0.3 \mathrm{~mm}, r_{s, 2}=0.52 \mathrm{~mm}$ and $Z_{v}=$ $Z_{C}=100 \Omega$. Finally the transfer impedances are equal to $Z_{T, 1}=0.005+j \omega l_{t} \Omega / m$ and $Z_{T, 2}=0.1+j \omega l_{t} \Omega / m$. 


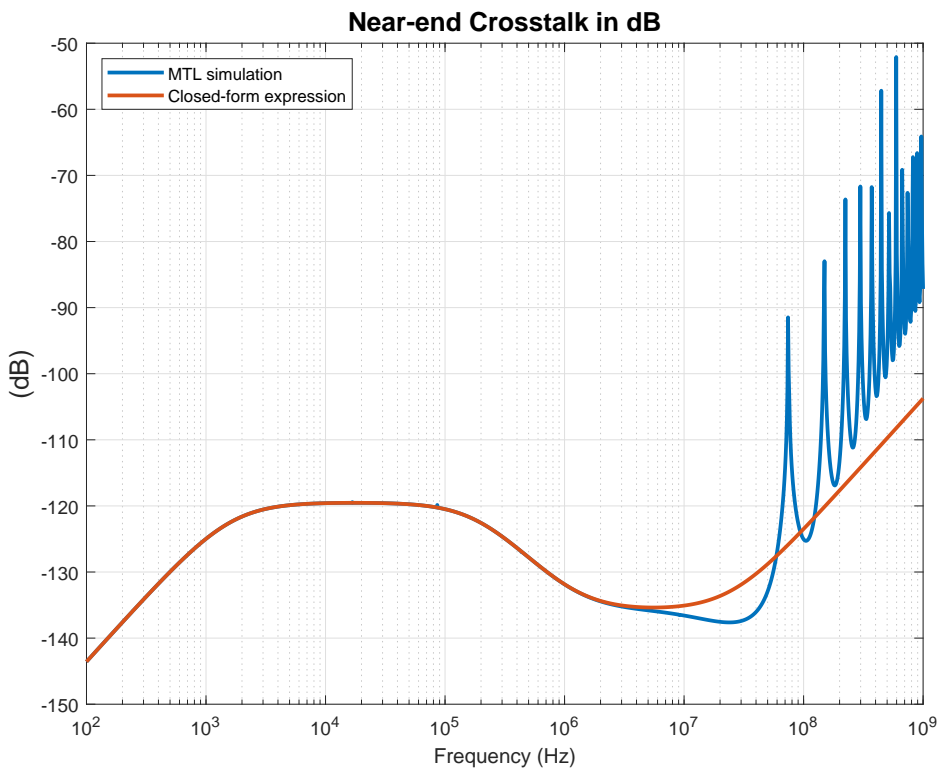

Figure 3.5: MTL simulations (blue) and the closed-form expression in (3.16) (red) of NEXT between an unshielded and a double shielded wire.

\subsubsection{Comparison to literature: one single shielded wire}

The configuration with two wires in which one of the wires carries a single shield has been covered in literature before (see for instance [36]). An illustration of this situation is given in Figure 3.6. If we follow the procedure to derive closed-form expressions as explained above, we find that the near-end crosstalk in this situation is equal to:

$$
\gamma_{N E} \approx \frac{j \omega \ell b Z_{T} \ln \left(1+4 h^{2} / d^{2}\right)}{4 Z_{C}\left[Z_{T}+j \omega b \ln \left(2 h / r_{S}\right)\right]}
$$

This expression coincides with results found in literature. Figure 3.7 again shows a good comparison between (3.17) and MTL simulations, in which we used the same parameter values as for Figure 3.5 (the single shield parameters are chosen equal to those of the outer shield in the previous situation).

\subsection{Generic crosstalk predictions}

The results of previous section show various regions that have different crosstalk behaviour with respect to frequency. This behaviour can be retraced and explained by further analysis of the closed-form expressions of NEXT. Several transition frequencies occur which are governed by shield self-inductance 


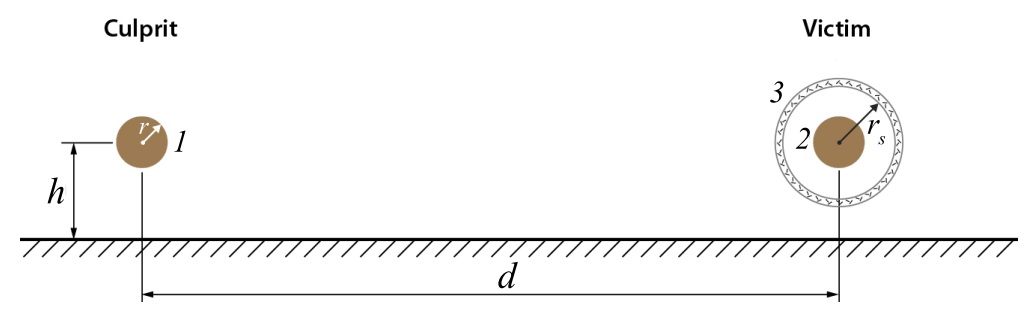

Figure 3.6: One unshielded wire and one wire carrying a single shield, both parallel above an infinite, perfectly conducting ground plane.

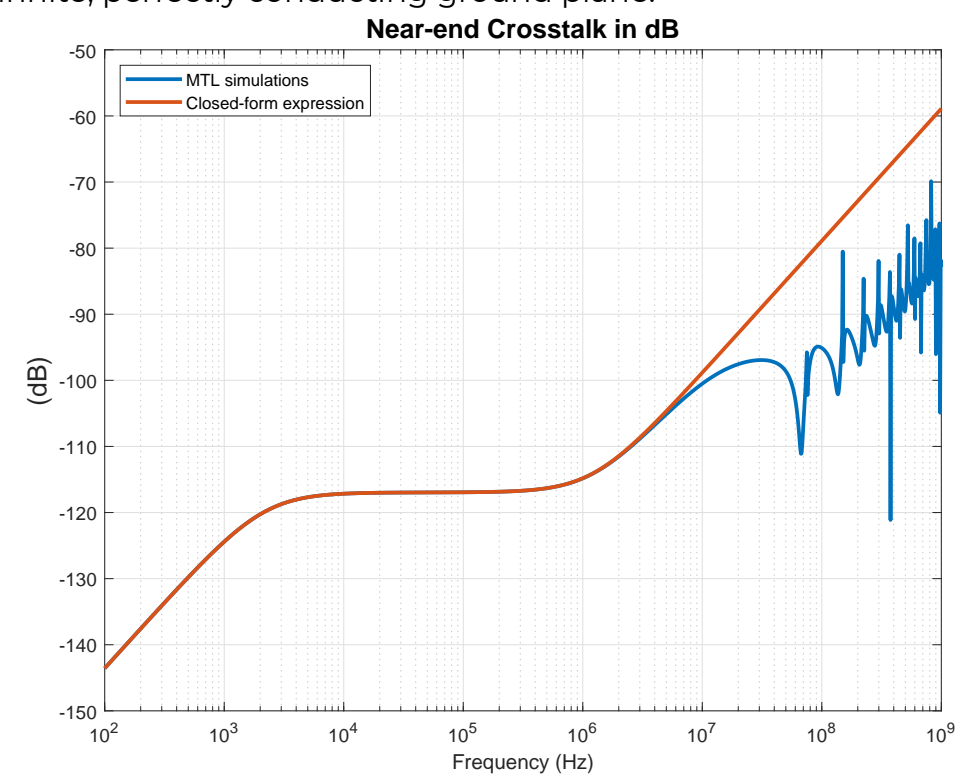

Figure 3.7: MTL simulations (blue) and the closed-form expression in (3.17)(red) of NEXT between an unshielded and a single shielded wire.

and transfer impedance phenomena. To find these dependencies and transitions we use equations (3.16) and (3.9) and investigate the orders of dependence with respect to frequency. This analysis leads to Figure 3.8 which illustrates the generic prediction of crosstalk with respect to frequency, as well as shielding parameters.

For the NEXT expressions derived in the previous section it holds that the leading order term in our low-frequency approximation equals the inductive coupling between two single wires above a ground plane (see Chapter 2). This holds for frequencies where both transfer impedance and shield inductance are negligible. In this first region of relatively low frequencies the behaviour is linear with respect to $\omega$ and the shields have no influence on inductive crosstalk levels. The shields only cause the capacitive crosstalk to vanish. In the following we will derive the other regions of different frequency 
dependence. Therefore, we assume the values used in simulations before, $5 \cdot 10^{-3} \Omega=R_{T, 1}<R_{T, 2}=10^{-1} \Omega$. Moreover $l_{t}=2 / \pi n F / m$, by which the self-inductance of the shield will play a role for lower frequencies than the inductance part of the transfer impedance. If values are different and the order of these inequalities changes the figures and transition frequencies below will also change, though the analysis procedure remains the same. Apart from the case of a single wire versus a double shielded wire, for which derivations of low-frequency NEXT were given in the previous section, also the generic predictions for crosstalk between two coaxes and between a single wire and a coax are given. The derivation of these is similar to what has been done for the double shielded wiring configuration.

\subsubsection{One double shielded versus one unshielded wire}

Analysis of (3.16) and Figure 3.5 reveals five different regions of crosstalk behaviour, which are separated by the following four transition frequencies:

$$
\omega_{k}= \begin{cases}\frac{2 \pi R_{T, 1}}{\mu_{0} \ln \left(2 h / r_{s, 1}\right)} & \text { for } k=1 \\ \frac{2 \pi R_{T, 2} \ln \left(2 h / r_{s, 1}\right)}{\mu_{0} \ln \left(r_{s, 2} / r_{s, 1}\right) \ln \left(2 h / r_{s, 2}\right)} & \text { for } k=2 \\ \frac{R_{T, k-2}}{l_{t}} & \text { for } k \in\{3,4\}\end{cases}
$$

These transition frequencies are also shown in Figure 3.8. The values of $\omega_{1}$ to $\omega_{4}$ separate the following crosstalk regions:

1. $\omega<\omega_{1}$

For all shielding configurations this first region obtains linear behaviour with respect to frequency and crosstalk levels are equal to inductive coupling when no shield is present. Any point on this line can be calculated by:

$$
\gamma_{N E}=\frac{j \omega \ell}{4 Z_{C}} \cdot b \ln \left(1+4 h^{2} / d^{2}\right) .
$$

2. $\omega_{1}<\omega<\omega_{2}$

In this region the self-inductance of the shield with the lowest transfer impedance starts to contribute. This frequency dependence cancels against the linear behaviour of the first region and therefore gives rise to a constant region in the crosstalk graph. Its value is equal to:

$$
\gamma_{N E}=\frac{\ell R_{T, 1}}{4 Z_{C}} \cdot \frac{\ln \left(1+4 h^{2} / d^{2}\right)}{\ln \left(2 h / r_{s, 1}\right)} .
$$




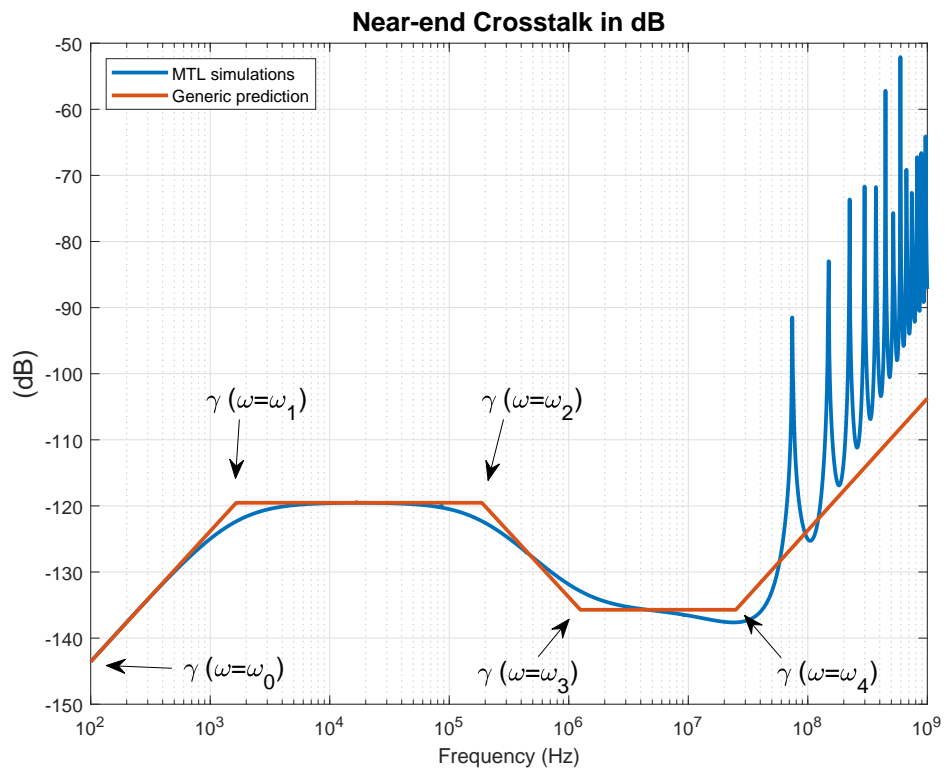

Figure 3.8: Generic prediction of crosstalk in the frequency domain (red) along with MTL simulations (blue) for the configuration of an unshielded versus a double shielded wire.

3. $\omega_{2}<\omega<\omega_{3}$

For these frequencies also the inductance of the second shield interacts. Then the crosstalk becomes inversely proportional to $\omega$. The values of this region can be calculated via:

$$
\gamma_{N E}=\frac{\ell R_{T, 1} R_{T, 2}}{4 b Z_{C}} \cdot \frac{\ln \left(1+4 h^{2} / d^{2}\right)}{\mathrm{j} \omega \ln \left(r_{s, 2} / r_{s, 1}\right) \ln \left(2 h / r_{s, 2}\right)} .
$$

4. $\omega_{3}<\omega<\omega_{4}$

Here the contribution of inductance of the transfer impedance of one of the shields becomes large in comparison to its resistance part. Then the frequency dependence in the numerator is cancelled and again a constant value appears, which is equal to:

$$
\gamma_{N E}=\frac{\ell l_{t} R_{T, 2}}{4 b Z_{C}} \cdot \frac{\ln \left(1+4 h^{2} / d^{2}\right)}{\ln \left(r_{s, 2} / r_{s, 1}\right) \ln \left(2 h / r_{s, 2}\right)} .
$$

5. $\omega>\omega_{4}$

In this last region also the inductance of the transfer impedance of the second shield becomes larger than its resistance. This ensures a linear 
increase of crosstalk with respect to frequency in the fifth region. The crosstalk formula now becomes:

$$
\gamma_{N E}=\frac{j \omega l l_{t}^{2}}{4 b Z_{C}} \cdot \frac{\ln \left(1+4 h^{2} / d^{2}\right)}{\ln \left(r_{s, 2} / r_{s, 1}\right) \ln \left(2 h / r_{s, 2}\right)} .
$$

In Figure 3.8 results of MTL simulations are shown together with the generic prediction. The results correspond very well, except for the fifth frequency region. This is a limitation to our model, since the chain matrices introduced in (2.10) are invalid for such high frequencies. This causes the fact that (3.10) already fails to hold.

Figure 3.9 shows a crosstalk measurement between an unshielded and double shielded wire compared to the corresponding generic prediction and MTL simulations. Due to the broad frequency range two separate generators had to be used, one from $100 \mathrm{~Hz}$ up to $1 \mathrm{MHz}$ and the other above $1 \mathrm{MHz}$. The two results are tied together at $1 \mathrm{MHz}$, indicated by the dash in the measurement trace. The culprit in this case was an AWG28 wire, while the double shielded wire was an RG223 cable, with $r_{s, 1}=1.74 \mathrm{~mm}, r_{s, 2}=2 \mathrm{~mm}, R_{T, 1}=16.2$ $\mathrm{m} \Omega, R_{T, 2}=18.4 \mathrm{~m} \Omega, l_{t, 1}=8 /(3 \pi) \mathrm{nH}, l_{t, 2}=3 / \pi \mathrm{nH}$ and inner wire radius equal to $0.45 \mathrm{~mm}$. The single wire was placed directly against the double shielded wire, yielding $d=3 \mathrm{~mm}$. The length of the cables was $77 \mathrm{~cm}$ and the height above ground $h=35.5 \mathrm{~mm}$. The measured results in Figure 3.9 show a very good match with the generic predictions. The difference of a few $\mathrm{dB}$ that is observed is likely to be caused by a mismatch in parameters for the double shielded wire in our model, compared to the actual parameters. For instance, the transfer resistance and inductance of the separate shields of this RG223 cable had to be estimated from transfer impedance measurements of the separate shields, which naturally comes with uncertainties. Finally, in the figure the fourth frequency region is actually there, but the differences between $\omega_{3}$ and $\omega_{4}$ are so small that this second plateau region is barely visible in the results.

\subsubsection{One single shielded versus one unshielded wire}

Consider the case in which the double shielded wire is replaced by a coax. Analysis of equation (3.17) yields a generic prediction for the near-end crosstalk comprising three frequency regions, which are separated by two transition frequencies:

$$
\omega_{k}= \begin{cases}\frac{2 \pi R_{T}}{\mu_{0} \ln \left(2 h / r_{s}\right)} & \text { for } k=1 \\ \frac{R_{T}}{l_{t}} & \text { for } k=2\end{cases}
$$

The values of $\omega_{1}$ and $\omega_{2}$ separate the following crosstalk regions: 
3.3. GENERIC CROSSTALK PREDICTIONS

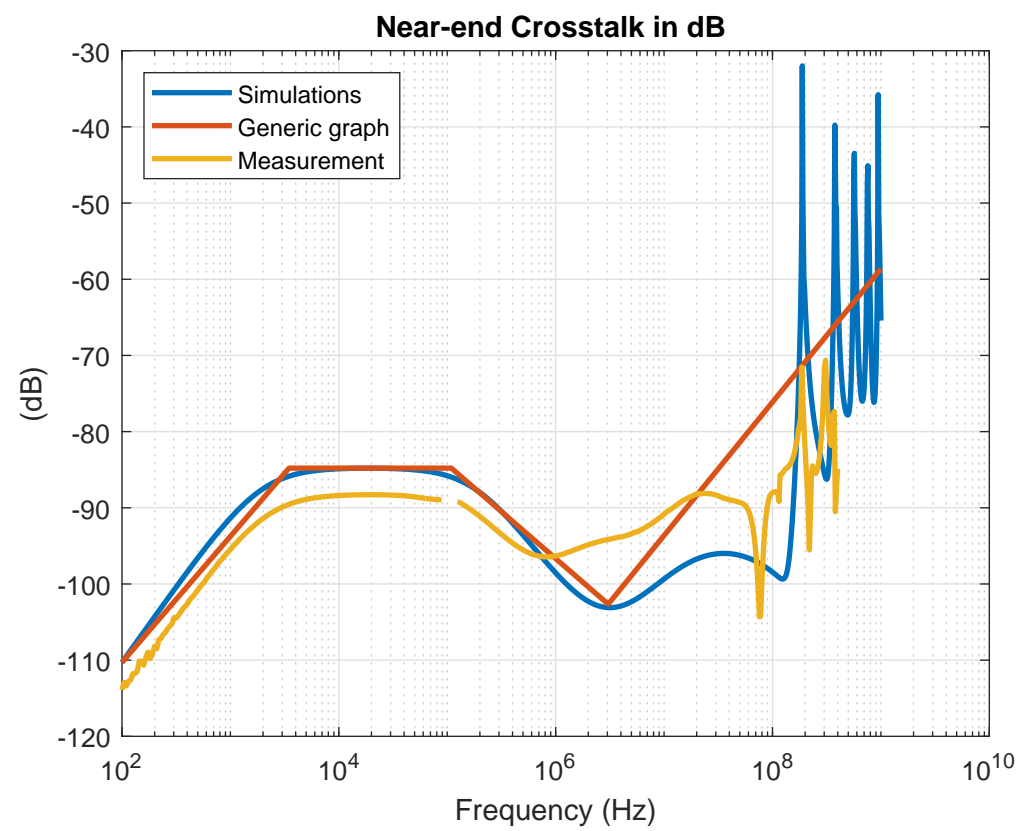

Figure 3.9: Comparison of the generic prediction (red line) of crosstalk between unshielded and double shielded wire versus MTL simulations (blue line) and measurements (yellow line).

1. $\omega<\omega_{1}$

The only effect of shielding is that capacitive coupling vanishes, and the crosstalk can be computed by equation (3.19).

2. $\omega_{1}<\omega<\omega_{2}$

The self-inductance of the single shield causes a plateau region, in which crosstalk can be computed by:

$$
\gamma_{N E}=\frac{\ell R_{T}}{4 Z_{C}} \cdot \frac{\ln \left(1+4 h^{2} / d^{2}\right)}{\ln \left(2 h / r_{S}\right)} .
$$

3. $\omega>\omega_{2}$

For these frequencies the transfer inductance is dominant over the transfer resistance, and crosstalk can be computed by:

$$
\gamma_{N E}=\frac{j \omega l l_{t}}{4 Z_{C}} \cdot \frac{\ln \left(1+4 h^{2} / d^{2}\right)}{\ln \left(r_{2 h} / r_{s}\right)} .
$$

Figure 3.10 compares the generic crosstalk predictions to MTL simulations and measurements for this cabling situation. The culprit was an AWG26 wire, 


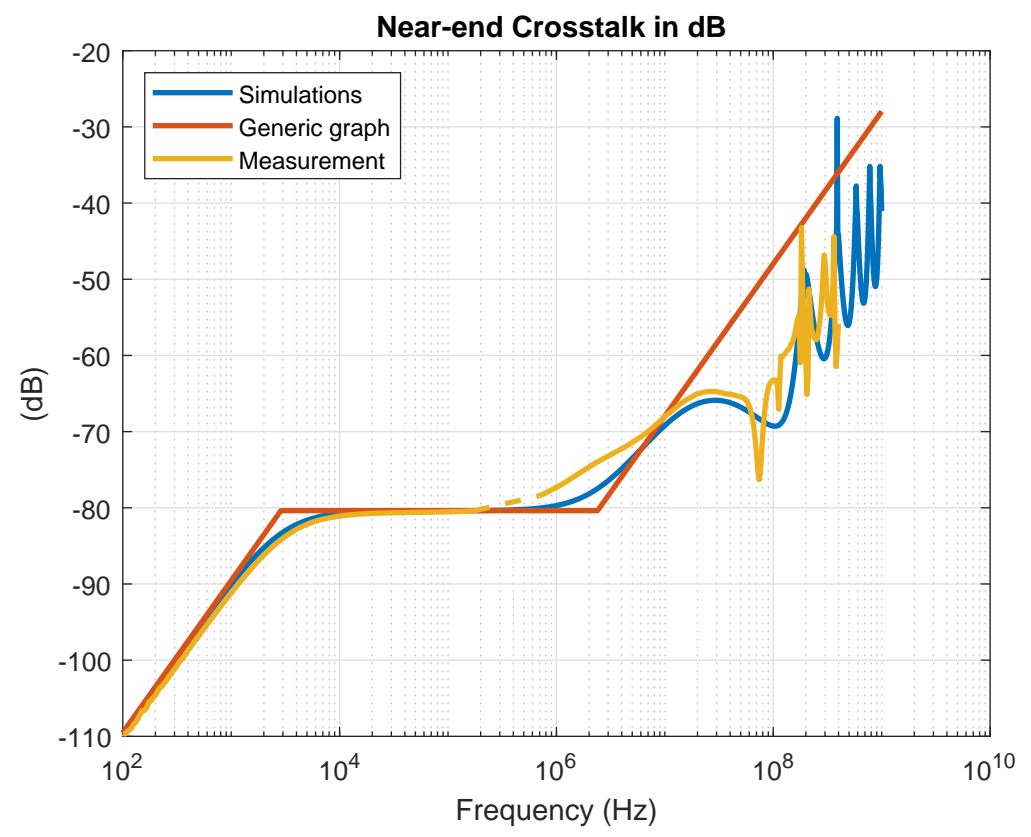

Figure 3.10: Comparison of the generic prediction (red line) of crosstalk between unshielded and shielded wire versus MTL simulations (blue line) and measurements (yellow line).

while the victim was an RG58 cable, of which the transfer resistance equalled $0.014 \Omega$, while the inductive part of the transfer impedance equalled $3 / \pi \mathrm{nH}$. The unshielded wire was placed directly against the coax cable, yielding a separation distance $d=2.9 \mathrm{~mm}$, while the height above ground was $h=45.3 \mathrm{~mm}$. The length of the cables was $\ell=77 \mathrm{~cm}$. The shield of the RG58 was connected to ground at both sides and the terminations of the inner conductor as well as the unshielded wire to ground were $50 \Omega$ at both sides. Finally, the radius of the shield was $1.7 \mathrm{~mm}$ and the radius of the inner core equalled $0.08 \mathrm{~mm}$. The results in Figure 3.10 show a very good match between the generic crosstalk prediction and the measurements. Even though the low-frequency expressions are not valid for the higher frequencies, for this case the generic crosstalk could even serve as an upper bound.

\subsubsection{Two single shielded wires}

The final case for which generic crosstalk prediction is analysed comprises two coaxes. This case consists of five frequency regions, which are separated by 
four transition frequencies:

$$
\omega_{k}= \begin{cases}\frac{2 \pi R_{T, 1}}{\mu_{0} \ln \left(2 h / r_{s, 1}\right)} & \text { for } k=1 \\ \frac{2 \pi R_{T, 2}}{\mu_{0} \ln \left(2 h / r_{s, 2}\right)} & \text { for } k=2 \\ \frac{R_{T, k-2}}{l_{t}} & \text { for } k \in\{3,4\}\end{cases}
$$

The values of $\omega_{1}$ to $\omega_{4}$ separate the following crosstalk regions:

1. $\omega<\omega_{1}$

The first frequency region remains unchanged an equal to equation (3.19).

2. $\omega_{1}<\omega<\omega_{2}$

In the second region the crosstalk is constant with frequency and computed by equation (3.20).

3. $\omega_{2}<\omega<\omega_{3}$

The second shield starts to interact as well, causing a decrease of crosstalk with frequency. Crosstalk values can be computed by:

$$
\gamma_{N E}=\frac{\ell R_{T, 1} R_{T, 2}}{4 b Z_{C}} \cdot \frac{\ln \left(1+4 h^{2} / d^{2}\right)}{j \omega \ln \left(2 h / r_{s, 1}\right) \ln \left(2 h / r_{S, 2}\right)} .
$$

4. $\omega_{3}<\omega<\omega_{4}$

The transfer inductance of one of the shields starts to dominate the transfer resistance, resulting in:

$$
\gamma_{N E}=\frac{\ell l_{t} R_{T, 2}}{4 b Z_{C}} \cdot \frac{\ln \left(1+4 h^{2} / d^{2}\right)}{\ln \left(2 h / r_{s, 1}\right) \ln \left(2 h / r_{s, 2}\right)} .
$$

5. $\omega>\omega_{4}$

Finally, also the second transfer inductance dominates the transfer resistance, yielding:

$$
\gamma_{N E}=\frac{j \omega l l_{t}^{2}}{4 b Z_{c}} \cdot \frac{\ln \left(1+4 h^{2} / d^{2}\right)}{\ln \left(2 h / r_{s, 1}\right) \ln \left(2 h / r_{s, 2}\right)}
$$

In Figure 3.11 the comparison between generic prediction, MTL simulation and measurement is shown for this situation of crosstalk between two shielded cables. In this case, both the culprit and the victim were an RG58 cable. The cables were separated by $d=20 \mathrm{~mm}$, while the height above ground was $h=$ $45.5 \mathrm{~mm}$. The length of the cables was $\ell=77 \mathrm{~cm}$, the shields were connected 
to ground at both sides and the termination of the wire to ground was $50 \Omega$. The measured results match very well with the generic crosstalk prediction. In this case, since the two coaxes are so similar, both plateaus have disappeared and the generic prediction actually consists of only three frequency regions.

\subsubsection{Comparison of various shielding configurations}

Generic crosstalk predictions have been derived for four different shielding configurations:

1. Two unshielded wires above a ground plane

2. One unshielded wire and one single shielded wire above a ground plane

3. Two single shielded wires above a ground plane

4. One unshielded wire and one double shielded wire above a ground plane.

Figure 3.12 compares the generic crosstalk predictions of these four wiring configurations. The shaded area in this figure indicates the frequency range in which the low-frequency assumptions that were used to derive the generic predictions fail to hold. For the unshielded case, the given result is still a valid upper bound, but for cases that involve shielding results in Figures 3.8-3.11 have shown that actual crosstalk levels can be higher than the generic predictions. For the simulations in Figure 3.12 the parameter values are equal to those given at the end of Section 3.2.1. For case 2 the shield radius and transfer impedance of the coax is equal to that of the outer shield of the double shielded wire. For case 3 the same holds for the shield radii, but for the transfer impedances the culprit shield is equal to the inner shield and the victim to the outer shield.

As stated in the previous section, the low-frequency behaviour of all situations is equal, except for a possibly significant part of capacitive crosstalk in the situation without shielding. The chosen loads result in mostly inductive crosstalk, though larger values for $Z_{v}$ and $Z_{c}$ could have made a bigger difference. For all shielded situations even the first constant regions are quite similar. The only difference in levels is caused by a smaller shield radius of the inner shield in the double shielded situation compared to the single shields in the other two configurations. This yields a slightly lower plateau value. The introduction of a second shield causes a temporary linear decrease and a second flat region before eventually crosstalk levels increase just like the configuration with one shield. The differences in transition frequency and constant value of situations 3 and 4 are caused by the difference in inductive coupling between the two shields. In the third case this is negligible by the fact that the separation distance of the wires is significantly larger than the radius of the shield. With the double shield this coupling is not significantly smaller than the selfinductances of the shields, which causes the differences observed in Figure 3.12. Evidently, the third and fourth transition frequencies are equal for these two situations, since the transfer impedances of the shields are chosen equal. 


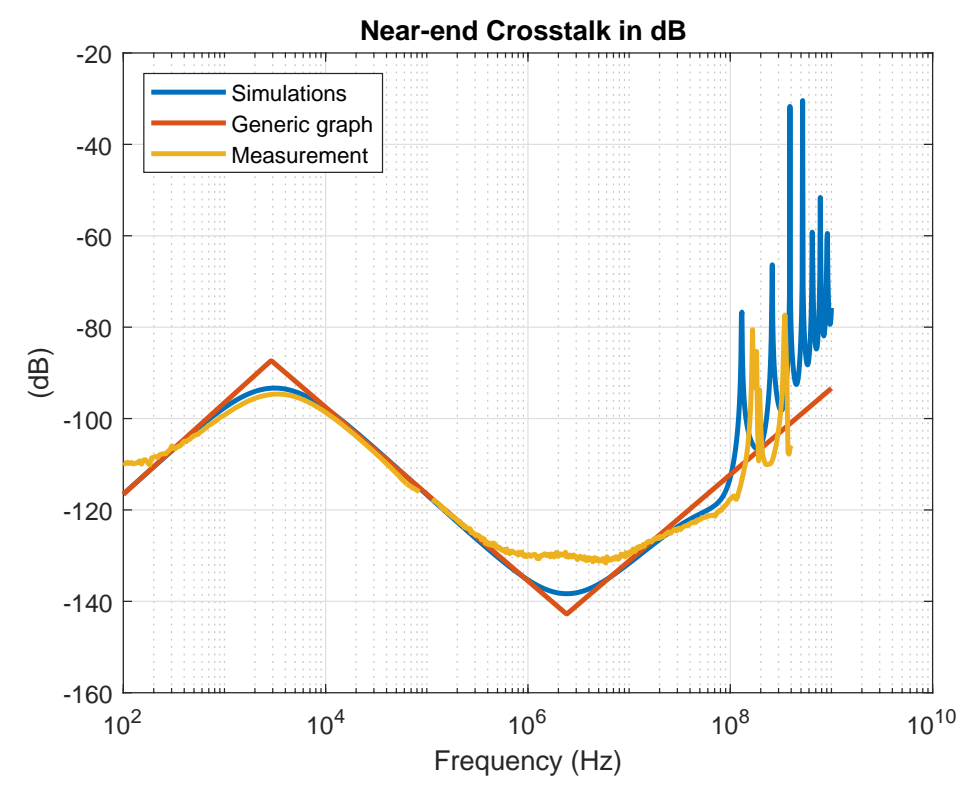

Figure 3.11: Comparison of the generic prediction (red line) of crosstalk between two shielded wires versus MTL simulations (blue line) and measurements (yellow line).

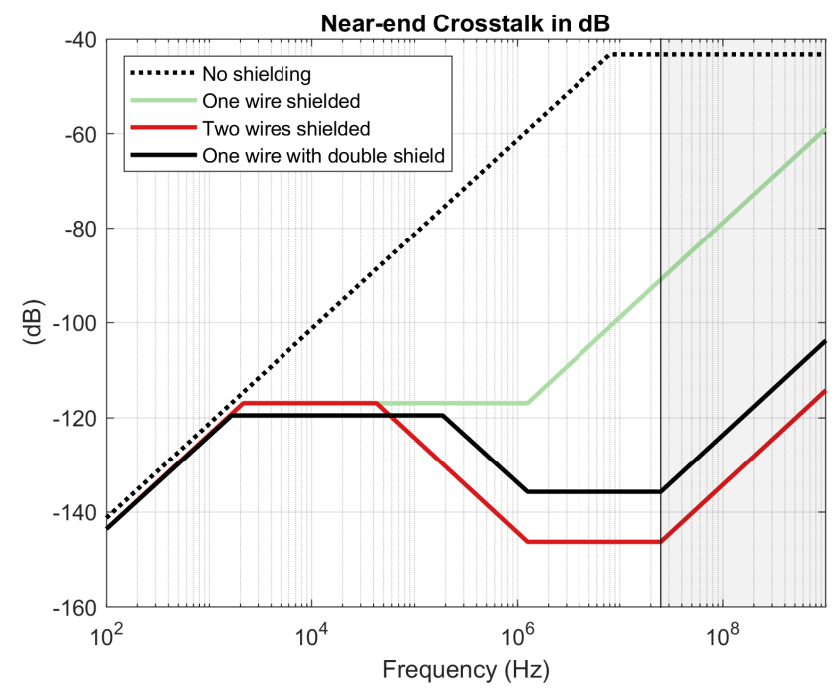

Figure 3.12: Comparison of the generic prediction of crosstalk in four different wiring configurations. The shaded area indicates the frequency region in which the lowfrequency assumptions used for derivation of the generic predictions fail to hold. 


\subsection{Summary}

A method to include transfer impedance parameters of multiple shields in crosstalk models has been described. The low-frequency solution of the MTL equations leads to closed-form expressions for near-end crosstalk which are valid as long as the cables are electrically short. These expressions explicitly contain geometrical parameters, as well as termination impedances and transfer impedance parameters. For the situation of one unshielded versus one double shielded wire the expression is given by (3.16). The analysis of this expression reveals regions of different frequency dependencies. For these regions generic crosstalk predictions have been presented, which describe in a practical way dependencies of the different regions and their crosstalk levels on shield resistance, shield inductance, termination impedance and geometrical parameters. Generic predictions where also given for crosstalk between two coaxes and crosstalk between a single wire and a coax.

Finally we compared generic crosstalk predictions of four different wiring configurations. This results in knowledge about the effects of shielding wires. The inclusion of a second shield causes crosstalk to decrease with frequency on a certain interval, which is followed by a second constant crosstalk level. Generic crosstalk prediction of different wiring configurations can be used to optimise shielding parameters to obtain certain crosstalk levels on specified frequency intervals. 


\section{Cables in the presence of composite ground planes}

Transmission lines are broadly covered in literature, especially when they are placed in free space with or without nearby conducting ground plane. The MTL model developed by Paul [5] is widely accepted for crosstalk analyses. Paul also includes losses in the p.u.l. resistance matrix of his MTL formulations. However, he clearly states the short-comings regarding the distribution of return currents in large, lossy ground planes. As introduced in Chapter 1 and confirmed by the expressions in Chapter 2, it is good practice to place cables close to a good conducting surface, as this generally reduces crosstalk levels. This chapter, which is based on [40], introduces efficient simulation models to compute crosstalk in cabling configurations that include lossy ground planes, such as CFRP, to evaluate the effects of replacing conducting aircraft structures by lossy alternatives to crosstalk behaviour.

Already in 1926, Carson investigated the influence of lossy soil on the behaviour of the transmission lines in a paper on wave propagation in overhead lines [41]. More specifically, he derived formulations which describe the distribution of electrical currents in a lossy ground, as well as an expression for the mutual impedance between two transmission lines that have a lossy ground as return. Since then, much research has focused on simulation models for transmission lines above lossy earth. Improvements to Carson's formulation have been made [42-45] and papers have been published on the use of these formulations in transient analysis of overhead lines above lossy grounds [4648]. Rachidi provides an extensive overview of field-to-TL coupling models that can also include the effects of lossy earth [49]. However, most papers focus on a lossy ground with infinite thickness (e.g. soil), as well as the transient (time-domain) analysis of transmission lines under the influence of incident EM fields and lightning strikes [49,50].

The focus in this chapter is on frequency domain simulations of crosstalk between cables in the presence of ground planes with finite conductivity and finite thickness. In [25] it was observed that skin depth and ground plane thickness are the main factors dictating a shift in crosstalk behaviour in the frequency domain. In the higher frequency range crosstalk in the presence 
of a CFRP ground plane is similar to that above good conducting ground and thus the presence of the ground plane decreases crosstalk. For lower frequencies crosstalk levels are hardly affected by the presence of CFRP. These observations in [25] were obtained from full-wave simulations and confirmed by measurements. Most commercially available full-wave solvers can model the effects of CFRP on crosstalk. However, such methods are computationally expensive and faster methods are desired, especially for industrial applications. The MTL equations are a widely used and much quicker method. An accurate and broadband numerical approach to estimate the p.u.l. parameters that includes skin effect, current crowding and semiconducting material is presented in [51]. However, this still requires the numerical MoM to solve the p.u.l. parameters.

Time-efficient broadband simulation methods that can provide an accurate first estimate of crosstalk in the presence of lossy ground planes are very useful for early risk assessment in EWIS design. Moreover, such methods can be used in cable bundle optimisation, as well as in sensitivity analysis with respect to designable parameters. This chapter proposes two novel methods to incorporate lossy ground planes, including corresponding skin and proximity effects, directly into the MTL equations. Both methods are efficient solutions that are easy to apprehend and implement. The first method makes use of a discretisation of the ground plane into cylindrical conductors. In [52] such a discretisation of a copper ground plane was used to analyse ground currents. The second method of this chapter utilises a ground impedance matrix. This impedance matrix is derived from an expression for mutual impedance of overhead lines above a stratified earth [44]. Both methods are applied to crosstalk between wire pairs above a CFRP or aluminium ground plane. Moreover, the method of discretised ground planes is also applied to cabling between two CFRP or aluminium ground planes. Results of these simulations are compared to full-wave MoM simulations with Feko [34] and to measured crosstalk.

Section 4.1 discusses two MTL models that include lossy ground planes. In Section 4.2 simulation results of two cases are shown and compared to fullwave simulations as well as measurements. The final section summarises the chapter.

\subsection{Transmission line models}

Equations (2.1) and (2.2) describe the evolution of the voltages and currents along the line. All voltages are defined with respect to the reference conductor and the return current of all conductors also flows in this reference conductor. For a Perfectly Electric Conducting (PEC) ground plane this return current is concentrated in a delta-peak directly below the conductor itself. However, if losses are present in the ground, these currents tend to spread out due to dispersion $[5,41]$, as is shown by the illustration in Figure 4.1. If we extend this 


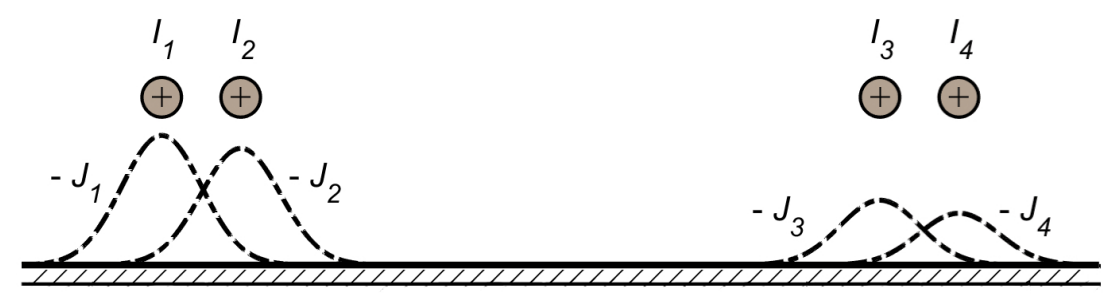

Figure 4.1: Illustration of (return) current distributions $J_{i}$ of the corresponding currents in the wires $I_{i}$ in a lossy ground plane.

further towards the case of no ground plane, the return currents will have vanished. Therefore, to model a CFRP ground plane with transmission line equations, we need to accommodate losses in the reference conductor. This chapter presents two models which can account for (return) current distributions in lossy ground planes. One method discretises the ground plane by a series of lossy wires, and the second implements a ground impedance matrix. In the following sections the two methods are described and explained based on the test case in Figure 4.2a. Two wire pairs are separated by a distance $d=20 \mathrm{~mm}$ and are at a height $h$ above a ground plane with thickness $t_{g}$. This ground plane is considered to be made of either aluminium with $t_{g}=1.5 \mathrm{~mm}$ or CFRP with $t_{g}=1.3 \mathrm{~mm}$. The intra-pair separation $a$ and the wire radius $r$ will be equal to $2.2 \mathrm{~mm}$ and $0.55 \mathrm{~mm}$, respectively, throughout the chapter. Both wire pairs are assumed to be terminated with a DM resistance $R_{d}=100 \Omega$ at both sides. Figure 4.2b also shows a case in which a second ground plane is placed above the wire pairs. This test case will be analysed in Section 4.2.

Assume a DM source is included in the terminations of the culprit wire pair. Consequently, a voltage will be induced in the terminations of the victim wire pair. Then differential-mode NEXT $\gamma_{N E, D M}$ can be defined as given in (2.3). In that equation $\mathbf{V}_{0}$ is the vector of which entry $i$ represents the voltage of conductor $i$ at the source side of the MTL with respect to the reference. $\mathbf{U}_{1}$ and $\mathbf{U}_{2}$ are vectors that are used to obtain the correct combination of conductor voltages in the numerator and denominator. For two wire pairs above a single infinite ground plane these would be defined as:

$$
\mathbf{U}_{1}=(-1,1,0,0)^{T}, \quad \mathbf{U}_{2}=(0,0,-1,1)^{T}
$$

FEXT can be defined in a similar way by using the voltages and currents at the other side of the transmission line. This chapter focuses only on NEXT, since the methods and models are analogously applied to FEXT.

Equation (2.3) shows that by solving the vector of voltages at the source side of the TL, crosstalk can be computed. These voltages can be solved by equations (2.5)-(2.9), depending on the termination network representation. In that solution, the p.u.l. matrices $\mathbf{C}, \mathbf{L}$ and $\mathbf{R}$ are required. The conductance 


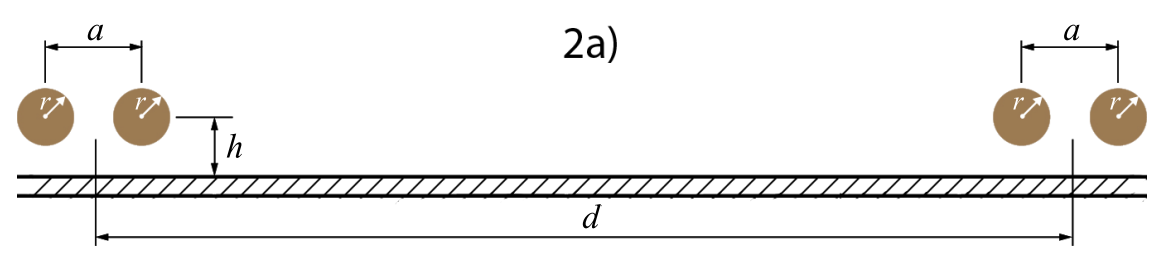

(a) Wire pairs above a single ground plane.

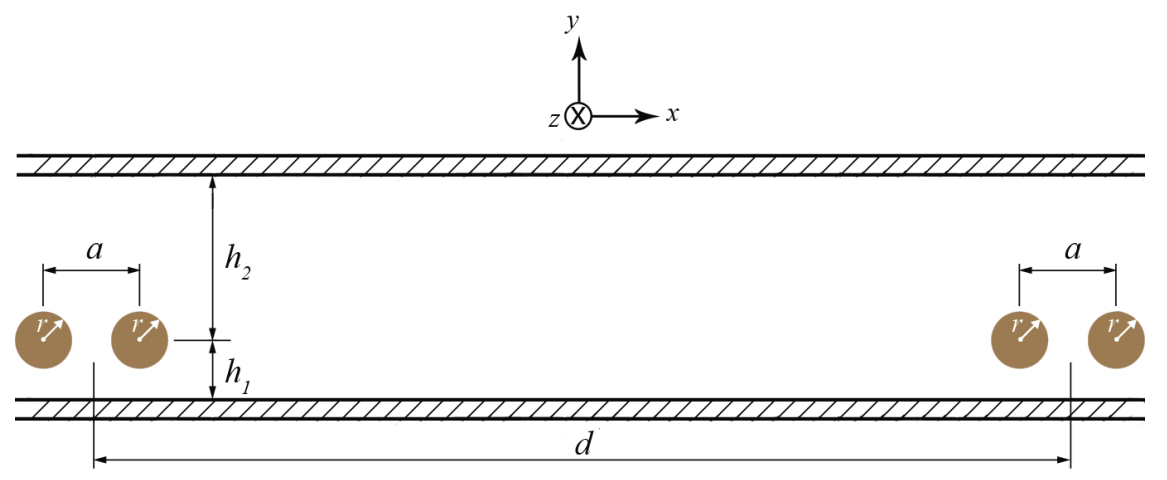

(b) Wire pairs between two ground planes.

Figure 4.2: Illustration of cross-section of crosstalk test cases.

G is assumed to be negligible. For an infinite PEC ground plane the approximate logarithmic expressions for p.u.l. capacitance and inductance as given by equation (2.21) can be used. Moreover, in the two new methods presented in the next two subsections that include lossy ground planes, for the self and mutual capacitances and inductances these same expressions can be used. The major differences that will be introduced are in resistance matrices and/or the termination impedance matrices.

\subsubsection{Discretised ground plane}

The first model presented in this section is an MTL model with discretised ground plane (MTL-DG). In this method, the ground plane is modelled as a series of cylindrical conductors parallel to the $z$-axis. The conventional MTL equations given by (2.1)-(2.2) can be used, however the dimensions of all matrices are increased with the number of conductors in the ground plane $n_{g}$. Moreover, the resistance matrix and termination matrices have to be adapted. Thus, to model the CFRP ground plane in the configuration of Figure 4.2a, instead of an infinite ground plane a finite ground plane is introduced, which will be represented by an array of $n_{g}$ adjacent cylindrical conductors. An example with a single layer of ground conductors, each with diameter $t_{g}$, is shown in Figure 4.3. In general, multiple layers of ground conductors can be used, as 


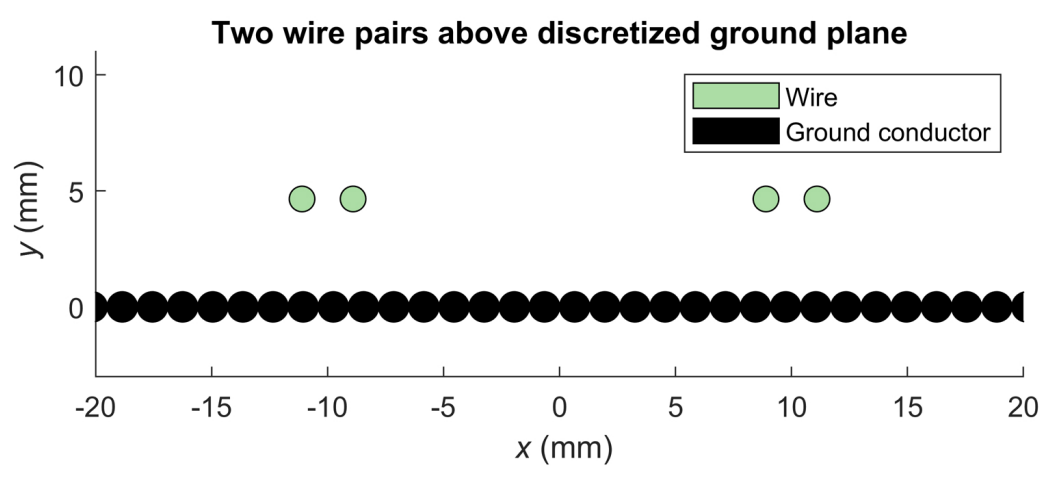

Figure 4.3: Illustration of (zoomed-in) cross-section for a cable configuration with two wire pairs above a discretised ground plane.

long as the diameters add up to $t_{g}$. Since in the measurements the ground planes are very large compared to the separation between the wire pairs, an infinite ground plane can be assumed. Therefore, in this chapter $n_{g}$ is also chosen large enough to mimic an infinite ground plane. Experiments to confirm this follow in Section 4.2. To avoid confusion, in the following the conductors of the ground plane will be referred to as ground conductors, while the four conductors in the wire pairs will be mentioned as wires.

With the replacement of the infinite ground plane by a (finite) array of ground conductors, there is no more natural choice for the reference conductor since, in MTL theory, the reference conductor carries a net current equal to the sum of the return currents from all other conductors. To resolve this issue, a "dummy" reference conductor is introduced far away from the wires and ground plane under consideration, in this thesis at $x=0 \mathrm{~m}$ and at $y=-$ $100 \mathrm{~m}$. Moreover, the termination network (shown in Figure 4.4) is designed to have all conductors floating with respect to the dummy reference. In calculations this dummy reference appears just as a placeholder for the voltage integrals, but it is a dead conductor. This means that due to the distance to the structure and termination impedances that approach infinity due to the floating dummy reference, the dummy carries no net (return) current and has no effect to the local behaviour of the wire pairs above ground. All currents flow locally in the wire pairs and the ground plane. Since the cross-section of the actual cabling remains small compared to the wavelength, TL theory can be applied [53]. The two wire pairs are terminated on both sides by the DM resistance $R_{d}=100 \Omega$.

To have all wires and ground conductors floating with respect to the dummy reference conductor, a Norton equivalent representation is required to incorporate the termination network into the current implementation of the MTL model. In theory, the ground conductors are short-circuited. However, 
Near-

end
Far-

end

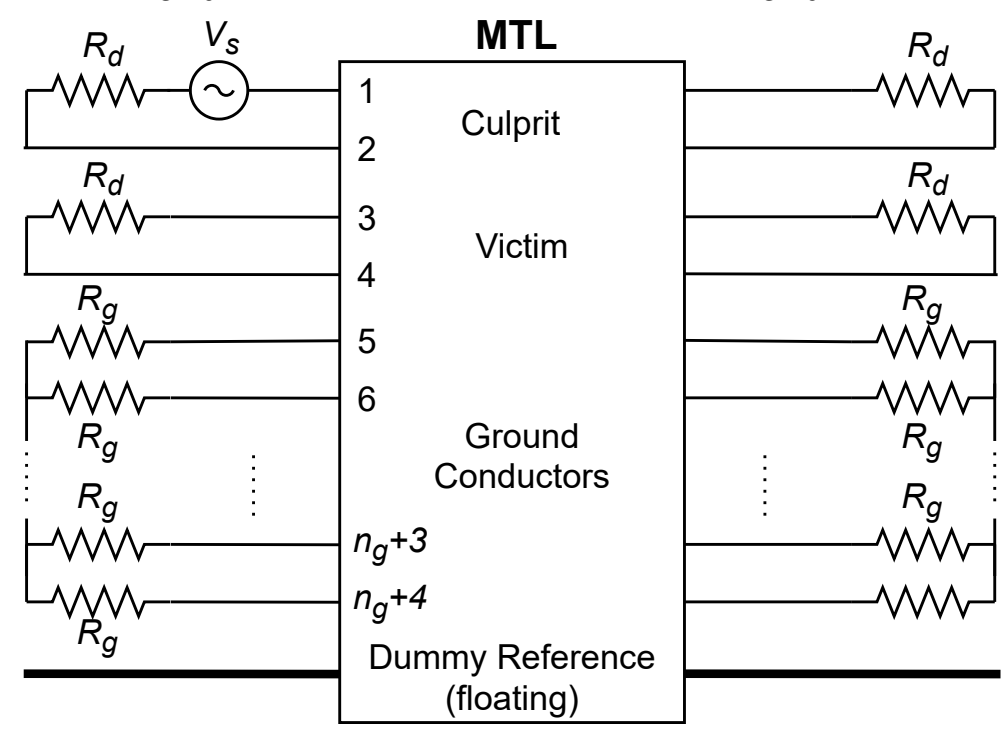

Figure 4.4: Termination schematics for the two wire pairs, terminated with DM resistance $R_{d}$, above a discretised ground with $n_{g}$ ground conductors that are connected to each other by the resistances $R_{g}$. Culprit terminations include a voltage source $V_{s}$.

this would create a singularity in the admittance matrices introduced in (4.2) below. To avoid this limitation, a small resistance $R_{g}=1 \mathrm{~m} \Omega$ (shown in 4.4) is used to mimic a short between all ground conductors. The value of $R_{g}$ is chosen such that it is negligible with respect to the losses in a ground conductor, which for a single layer of ground conductors equals $36 \Omega$ at $0.1 \mathrm{MHz}$ (computed by (4.5)). In this way $R_{g}$ does not affect crosstalk results and allows for current distribution through the ground plane. This results in the following admittance matrices at source and load side $\mathbf{Y}_{S}$ and $\mathbf{Y}_{L}$ :

$$
\begin{aligned}
& \mathbf{Y}_{S}=\mathbf{Y}_{L}=\left[\begin{array}{cc}
\mathbf{Y}_{N} & \mathbf{0} \\
\mathbf{0} & \mathbf{Y}_{\hat{g}}
\end{array}\right] \\
& \mathbf{Y}_{N}=\left[\begin{array}{cc}
\mathbf{Y}_{C} & \mathbf{0} \\
\mathbf{0} & \mathbf{Y}_{V}
\end{array}\right], \quad \mathbf{Y}_{k}=\left[\begin{array}{cc}
R_{d, k}^{-1} & -R_{d, k}^{-1} \\
-R_{d, k}^{-1} & R_{d, k}^{-1}
\end{array}\right] \\
& \mathbf{Y}_{\hat{g}}=\frac{1}{\left(1+\frac{1}{n_{g}-1}\right) R_{g}} \mathbf{1}_{n_{g}}-\frac{1}{n_{g} R_{g}} \mathbf{1}_{n_{g}}^{\prime} .
\end{aligned}
$$

Here $\mathbf{Y}_{N}$ contains the wire pair terminations and $\mathbf{Y}_{\hat{g}}$ the ground conductor terminations, $\mathbf{1}_{m}$ represents the $m \times m$ identity matrix and $\mathbf{1}_{m}^{\prime}$ the matrix with ones on all non-diagonal elements. The subscript $k$ can be $c$ for culprit and $v$ 
for victim.

To include the ground current distribution and skin effect in the MTL model the analytical resistance of cylindrical conductors with finite conductivity is included in the resistance matrix for all ground conductors. This yields a p.u.l. impedance matrix equal to:

$$
\hat{\mathbf{z}}=\mathbf{R}_{w}+\mathbf{Z}_{g}+\mathbf{j} \omega \mathbf{L}_{w}
$$

in which matrix $\mathbf{L}_{w}$ contains the self and mutual inductances of the entire MTL (including far-away reference). The elements of this inductance matrix are computed by (2.35) and the capacitance matrix by (2.27). In this case, the subscript 0 in (2.35) refers to the dummy reference. The conductor radius $r_{i}$ is either the wire radius ( $r$ in Figure 4.2) or the ground conductor radius. The radius of the dummy reference $r_{0}$ is chosen equal to the wire radius. In (4.3) the matrix $\mathbf{R}_{w}$ contains the resistances of the wires that form the two wire pairs. However, in this chapter the resistances in the wires are neglected, resulting in $\mathbf{R}_{w}=\mathbf{0}$. Finally, $\mathbf{Z}_{g}$ contains the resistances and inductances of the conductors that form the ground plane, for which the formulations described by Paul are used [5]:

$$
\mathbf{z}_{g}=z_{g} \mathbf{1}_{n_{g}}, \quad z_{g}=r_{g}+\mathrm{j} \omega l_{g}
$$

Here:

$$
\begin{aligned}
& r_{g}=\frac{2 q}{\sigma_{g d} \pi d_{g}^{2}}\left(\frac{\operatorname{ber}(q) \operatorname{bei}^{\prime}(q)-\operatorname{bei}(q) \operatorname{ber}^{\prime}(q)}{\left(\operatorname{bei}^{\prime}(q)\right)^{2}+\left(\operatorname{ber}^{\prime}(q)\right)^{2}}\right) \\
& l_{g}=\frac{4 \mu_{0}}{8 \pi q}\left(\frac{\operatorname{bei}(q) \operatorname{bei}^{\prime}(q)+\operatorname{ber}(q) \operatorname{ber}^{\prime}(q)}{\left(\operatorname{bei}^{\prime}(q)\right)^{2}+\left(\operatorname{ber}^{\prime}(q)\right)^{2}}\right), \quad q=\sqrt{2} \frac{d_{g}}{2 \delta} .
\end{aligned}
$$

Here ber and bei are Kelvin functions, $d_{g}$ is the ground conductor diameter, which for the discretisation in Figure 4.3 is equal to $t_{g}$, and $\delta$ is the skin depth, given by:

$$
\delta=\frac{1}{\sqrt{\pi f \mu_{0} \sigma_{g d}}}, \quad \sigma_{g d}=\frac{4 \sigma_{g}}{\pi} .
$$

Here the free space permeability $\mu_{0}$ is used since there is no magnetic material, and $\sigma_{g}$ is the conductivity of the ground plane. The value $\sigma_{g d}$ is the conductivity used for the ground conductors, which contains a correction factor to account for the difference in area that is missing since we are discretising a rectangular area by circles.

\subsubsection{Ground impedance}

The second method to incorporate the losses and current distribution in the transmission line models is to include a ground impedance matrix into the MTL equations (MTL-GI). The transmission line model remains as given by (2.1) 
and (2.2). In the MTL-GI method, as opposed to the MTL-DG method, the ground plane can be taken as infinite. Therefore, the configuration of Figure $4.2 a$ results in $4 \times 4$ inductance and capacitance matrix of which the elements are computed by equation (2.35) and (2.27). The conductance matrix is still assumed to be zero and the p.u.l. impedance matrix is again defined as given in (4.3), this time resulting in a $4 \times 4$ matrix. Analogous to the MTL-DG method we neglect the resistances in the wires. The effects of CFRP should be included in $\mathbf{Z}_{g}$. There is extensive literature about such impedance matrices that include the losses of soil into transmission line equations. Carson [41] was one of the first ones to derive an equation for ground impedance in which the soil was represented by a lossy half-space. By adapting the formulation for ground impedance of a stratified soil that is given in [44] to the case of a ground plane with finite thickness and conductivity, the ground impedance matrix for MTL$\mathrm{Gl}$ is given by:

$$
\left[\mathbf{z}_{g}\right]_{i j}=\frac{j \omega \mu_{0}}{\pi} \int_{0}^{\infty} \frac{\cos \left(\alpha\left(x_{i}-x_{j}\right)\right) \mathrm{e}^{-\alpha\left(h_{i}+h_{j}\right)}}{\alpha+A} d \alpha
$$

Here:

$$
\begin{aligned}
A & =n_{2}\left[\frac{n_{1}-n_{2}+\left(n_{1}+n_{2}\right) \mathrm{e}^{2 t_{g} n_{2}}}{n_{2}-n_{1}+\left(n_{1}+n_{2}\right) \mathrm{e}^{2 t_{g} n_{2}}}\right] \\
n_{1} & =\sqrt{\alpha^{2}+\mathrm{j} \omega \mu_{0}}, \quad n_{2}=\sqrt{\alpha^{2}+\mathrm{j} \omega \mu_{0} \sigma_{g}} .
\end{aligned}
$$

Here $x_{i}$ gives the $x$ position of wire $i$, while $h_{i}$ is the height of wire $i$ above the ground plane. Apart from this adaptation, the MTL equations remain unchanged and the termination admittance matrices are defined by:

$$
\mathbf{Y}_{S}=\mathbf{Y}_{L}=\left[\begin{array}{cc}
\mathbf{Y}_{C} & \mathbf{0} \\
\mathbf{0} & \mathbf{Y}_{V}
\end{array}\right] \text {. }
$$

\subsection{Results}

In this section crosstalk results from the two MTL models presented in Section 4.1 are compared to full-wave simulations obtained with Feko and to measurements. The comparison is performed for two configurations. At first, the configuration of two wire pairs above a single ground plane, as shown in Figure 4.2a, is computed by both the MTL-DG and the MTL-GI method and compared to Feko simulations and measurements. Thereafter, the MTL-DG method is applied to a configuration with wire pairs between two ground planes, shown in Figure 4.2b. The MTL-DG method can be readily adapted to this second case, due to its flexibility and ease of use. It only requires the inclusion of a higher number of ground conductors. The MTL-GI method is not directly applicable to this case with two ground planes, unless for instance a Laplace solver is incorporated and (4.7) is modified accordingly. 
All measurements are performed with the combination of a signal (tracking) generator as source and a spectrum analyser to measure the victim voltage (see Figure 4.5). As in Chapter 2 the balanced wire pairs are connected to the unbalanced measurement equipment using baluns. These baluns can be used in the frequency range of $100 \mathrm{kHz}-440 \mathrm{MHz}$. Their frequency-dependent behaviour is calibrated out of the measurements. Rohacell spacers with low loss and relative permittivity nearly equal to 1 are used to ensure fixed separation distances and heights of the ground plane along the length of the transmission line, which equals $1.8 \mathrm{~m}$. The ground planes used in the measurements were $1.8 \mathrm{~m}$ in length and $1 \mathrm{~m}$ in width. Their thickness equalled $1.3 \mathrm{~mm}$ and $1.5 \mathrm{~mm}$ for the CFRP and aluminium grounds, respectively. At the end of the transmission lines, the wire pairs are separated from each other and connected to the balun boards (see Figure 4.5b). Connections between the baluns and the ground planes are established by screws through the ground plane, completed with conductive glue for tighter fastening of the screws. This results in a CM impedance of $450 \Omega$, which is high enough to use in simulations only DM terminations [25]. In simulations the CFRP is modelled with an isotropic equivalent conductivity, which was found to be valid in [24, 25,54]. For our CFRP planes, this equivalent conductivity equals $16.5 \mathrm{kS} / \mathrm{m}$ [25].

In the simulations, a homogeneous dielectric with an effective relative permittivity equal to 2.5 is assumed to account for insulation around the wires and foam spacers. Feko simulations are performed by making use of an isotropic thin dielectric layer to which CFRP properties are asserted.

\subsubsection{Single ground plane}

Figures 4.6 to 4.9 show results for crosstalk between wire pairs without nearby ground plane, as well as for wire pairs at a height $h=4 \mathrm{~mm}$ above a ground plane (cross-section of the latter shown in Figure 4.2a). Figure 4.6 shows a comparison between near-end crosstalk computed by various simulation models. Results are given for three configurations: wire pairs in without ground plane, wire pairs above an aluminium ground plane and wire pairs above a CFRP ground plane. All three configurations are simulated with Feko and the results are shown by the solid lines in Figure 4.6. With classic MTL simulations, in which the logarithmic (wide-separation) expressions by Paul (equations (2.21) and (2.35)) are used for inductance and capacitance, the dotted lines in Figure 4.6 are obtained.

The results in Figure 4.6 confirm the well-known phenomenon that crosstalk decreases for cables that are placed close to a metallic ground plane, when compared to wire pairs without nearby ground plane. For these two cases MTL results are equal to those generated by Feko. The MTL-DG method introduced in the previous section yields levels shown by the plus signs in Figure 4.6. With this method both the aluminium ground plane case and the CFRP ground plane case are shown and results show a perfect match to those obtained with Feko. Finally, results obtained with MTL-GI are given by the cir- 

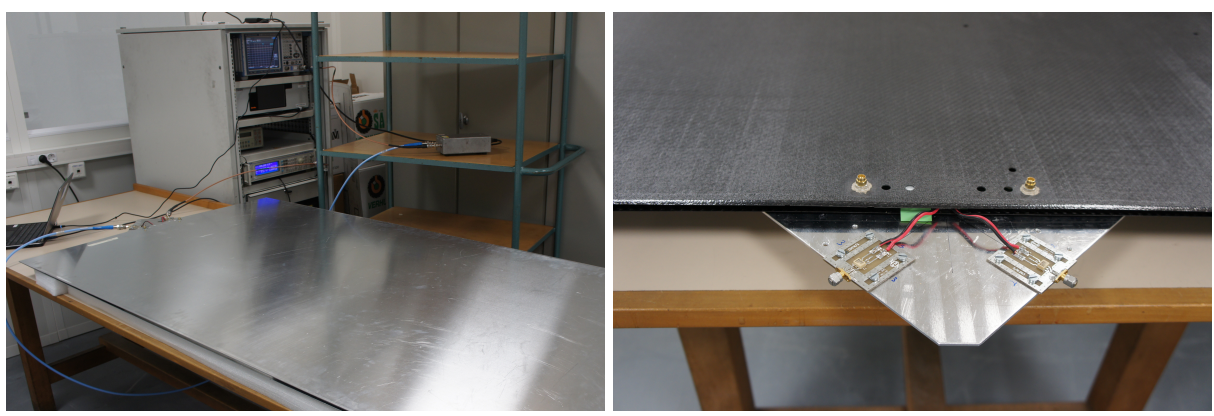

(a) Wire pairs between two aluminium (b) Wire pairs between two CFRP ground planes - overview of setup ground planes - close-up of terminawith spectrum analyser, generator and tion to baluns.

preamplifier.

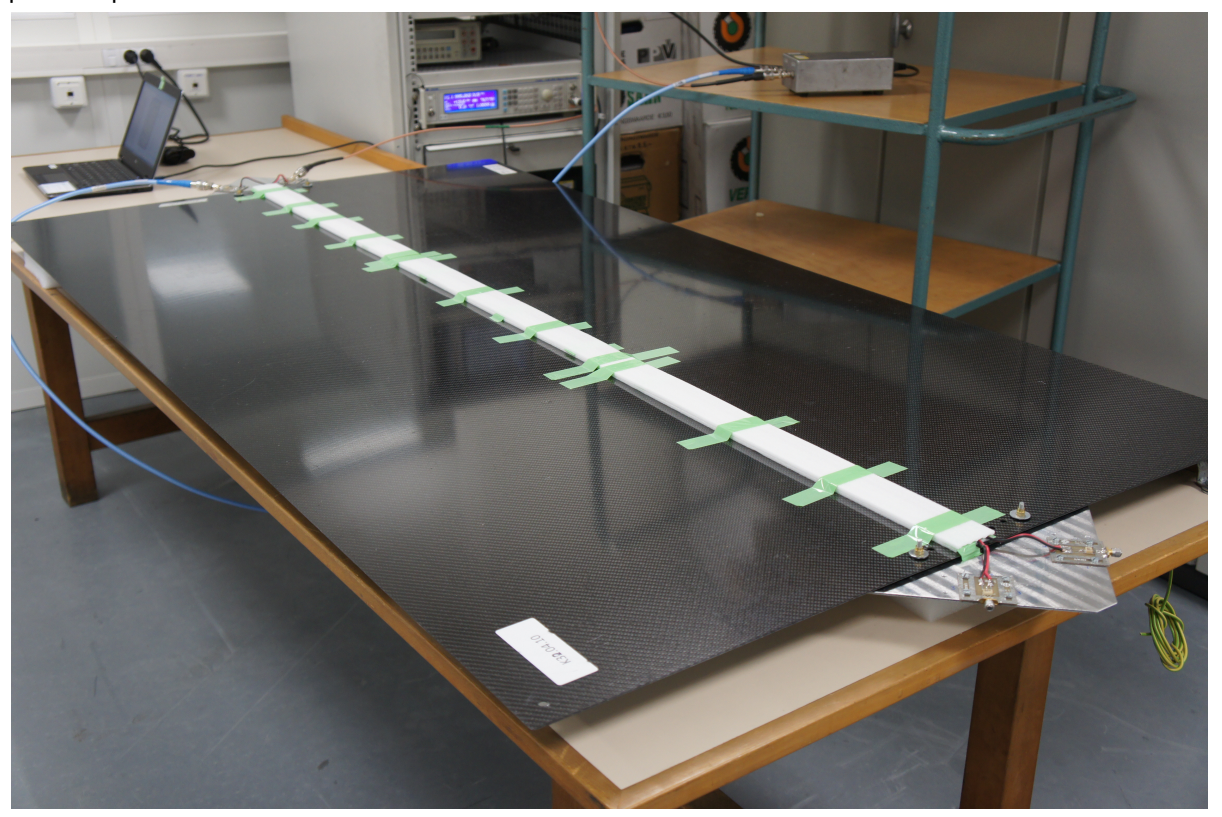

(c) Wire pairs above a single CFRP ground plane.

Figure 4.5: Pictures of the crosstalk measurement setup. 


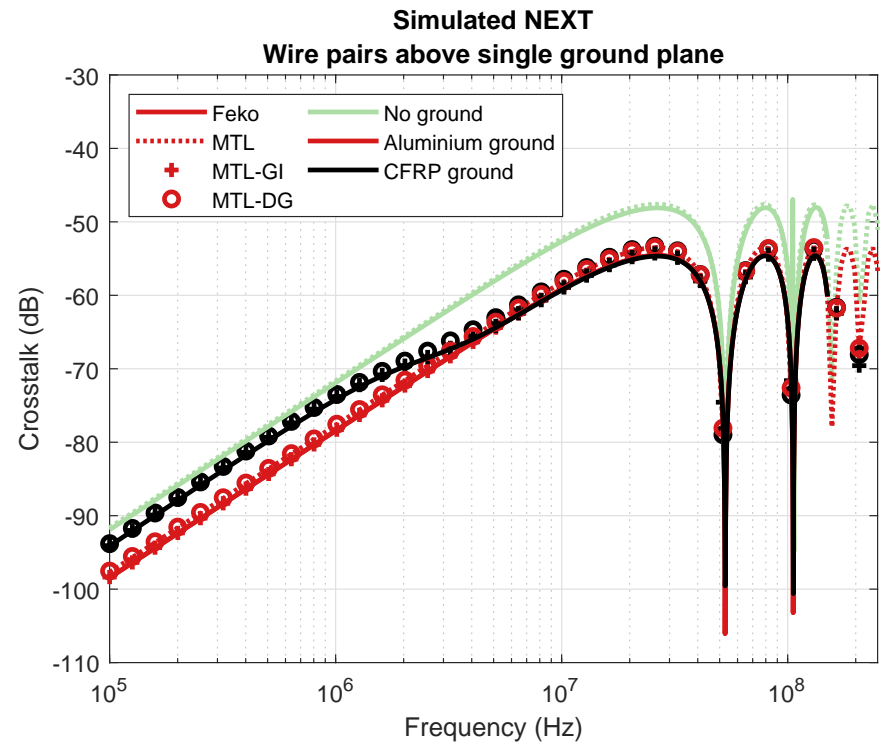

Figure 4.6: Simulated NEXT between two wire pairs in three different configurations. Solid lines are obtained with Feko, dotted lines with the analytic MTL equations. Circles give results computed by MTL-GI, while the plus signs show results for the MTL-DG method.

Simulated NEXT

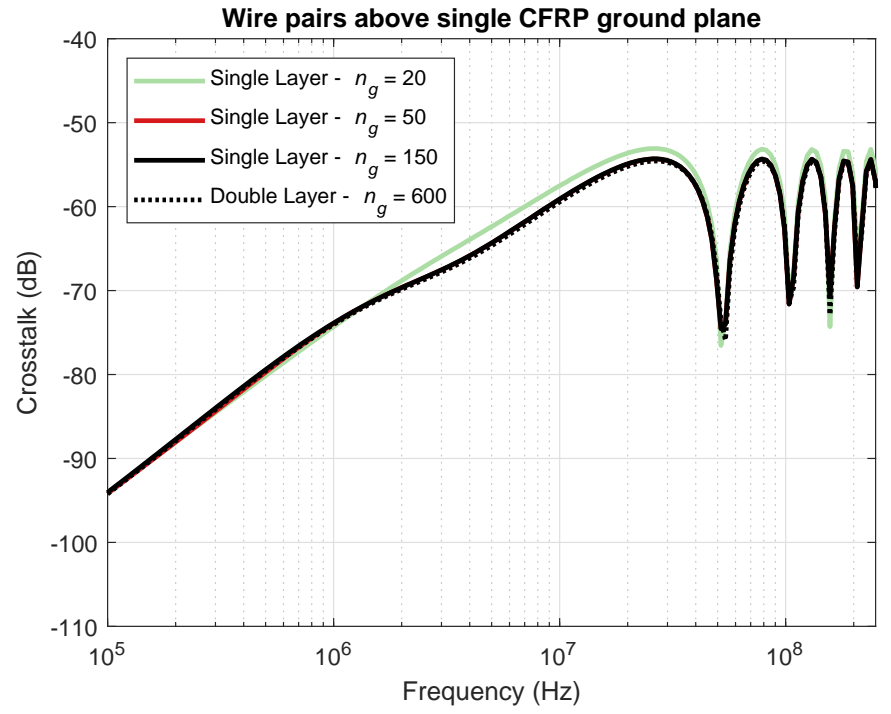

Figure 4.7: Simulated NEXT (MTL-DG) for wire pairs above a single CFRP ground plane. Comparisons are made between three different sizes of a single layer ground plane and a double layered ground plane. 
cles. Again, these results match perfectly to the other simulation methods.

Various discretisation levels might be used in the MTL-DG method. Figure 4.7 shows a comparison of results for a CFRP ground plane with a single layer and 3 different values for $n_{g}$, as well as a discretisation with two layers of wires. In all cases, the ground conductors are touching. Increasing $n_{g}$ in a single layer will therefore increase the ground plane size. Introducing a second layer of ground conductors might increase accuracy, but also increases computation time. From Figure 4.7 it can be concluded that a single layer with 50 ground conductors is sufficient to represent an infinite ground plane, for the case under consideration. Therefore, in all MTL-DG simulations in this chapter a single layer with $n_{g}=50$ conductors is used.

Concluding, similar results are obtained by all three simulation methods. However, simulation times are very different. Fine meshing is needed on a ground plane below a transmission line in order to accurately represent the characteristic impedance in Feko. Therefore, simulations with Feko depend on the wire height and vary for $h=4 \mathrm{~mm}$ to $1 \mathrm{~mm}$ from 60 seconds to 1060 seconds for a single-frequency computation, when using 10 cores on a Linux server (Intel(R) Xeon(R) Gold $6148 \mathrm{CPU} @ 2.40 \mathrm{GHz}, 80$ cores available). At the same time, the MTL-DG and MTL-GI methods take only 6.5 and 3 seconds, respectively, for computing 200 frequencies on a simple laptop (1 core on Intel i5 processor, 8GB RAM). Therefore, simulation time for a single frequency computation is improved by a factor of 1,900 - 70,000. When comparing the two novel MTL methods, MTL-GI is slightly more time-efficient and enables actual infinite ground plane simulation. However, as illustrated also by the case in Section 4.2.2, the MTL-DG method provides more flexibility.

Figure 4.8 shows the comparison of the MTL simulations with measured NEXT. For the ground plane cases the MTL-GI method is shown. Some uncertainties in the measurement setup cause the measured crosstalk curve to have a slope slightly less than the expected $20 \mathrm{~dB} /$ decade, which causes slight differences in the 0.1-0.3 MHz range. However, in general the comparison between simulations and measurements is good. The simulation results clearly show that CFRP affects crosstalk behaviour as stated in [25]. There is a clear distinction between low and high-frequency behaviour, in which both the conductivity and thickness of the ground plane are important parameters. The distinction is determined by the field penetration in the CFRP material [55]. At low frequencies, for which the skin depth is much larger than the thickness of the ground plane, the electromagnetic fields tend to distribute throughout the ground plane and consequently the currents distribute in depth and transversal directions of the ground. At high frequencies, for which the skin depth is small compared to the ground plane thickness, the fields penetrate only the top part of the ground plane given by one skin depth and currents concentrate at the surface and below the wires. For the good conducting aluminium ground planes this high-frequency effect holds throughout the entire frequency range considered in this study.

These observations are confirmed by results shown in figures 4.9 and 4.10. 


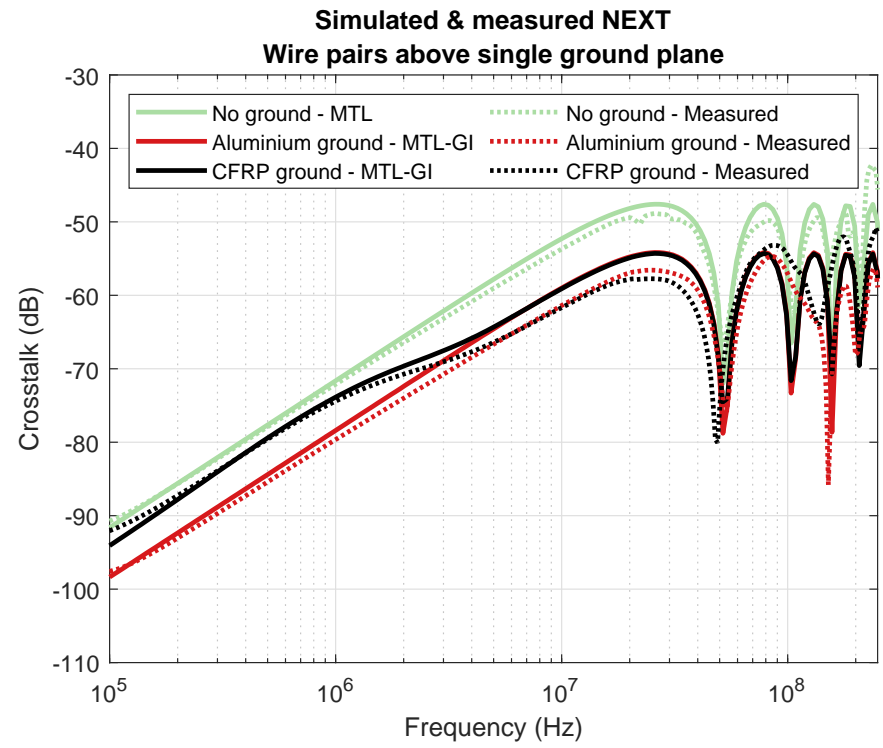

Figure 4.8: Comparison between measured and simulated NEXT for wire pairs without ground plane, or above a single ground plane. Solid lines are results of NEXT simulations (also shown in Figure 4.6, analytic MTL without ground plane and MTL-GI for the ground plane cases). The dotted lines are measured crosstalk results.

Simulated NEXT

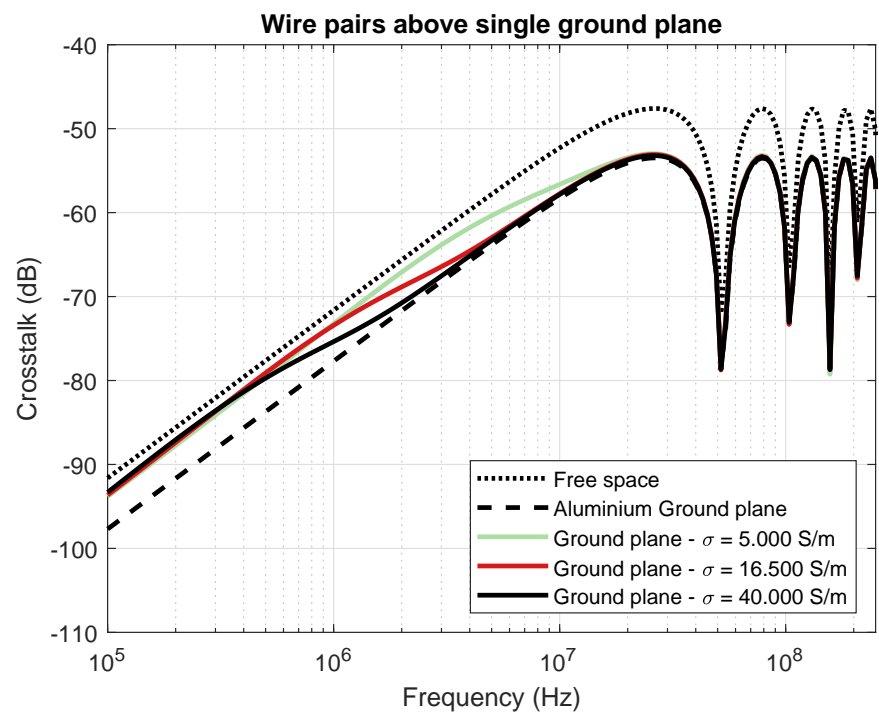

Figure 4.9: Simulated (MTL-DG method) NEXT between two wire pairs above a single ground plane with different conductivities. MTL results with and without PEC ground plane are given as reference. 
Figure 4.9 shows that for lower conductivity, the transition from free-spacelike to PEC-like behaviour occurs at a higher frequency than for higher conductivity. For the conductivity of our measured CFRP, i.e. $16.5 \mathrm{kS} / \mathrm{m}$, Figure 4.10 also shows current distributions in the ground plane. To better show current distributions, a ground plane discretisation with two layers of ground conductors is used for the computation of results in Figure 4.10. A big advantage of the MTL-DG method is that these current distributions are readily available after solving the MTL equations. For all subplots the left wire pair is the culprit, and the magnitudes of ground plane currents are shown in correspondence with the colour bar. The two upper subfigures of Figure 4.10 show the current distributions in a CFRP ground plane, while the two bottom subfigures are for an aluminium ground plane. Indeed, good field penetration in the CFRP for the lower frequencies causes the currents to distribute wider across the ground plane, while they become more concentrated below the current-driving wires for higher frequencies, showcasing proximity effects. In aluminium, the currents in the ground plane are concentrated below the wires in the entire frequency range considered in this study.

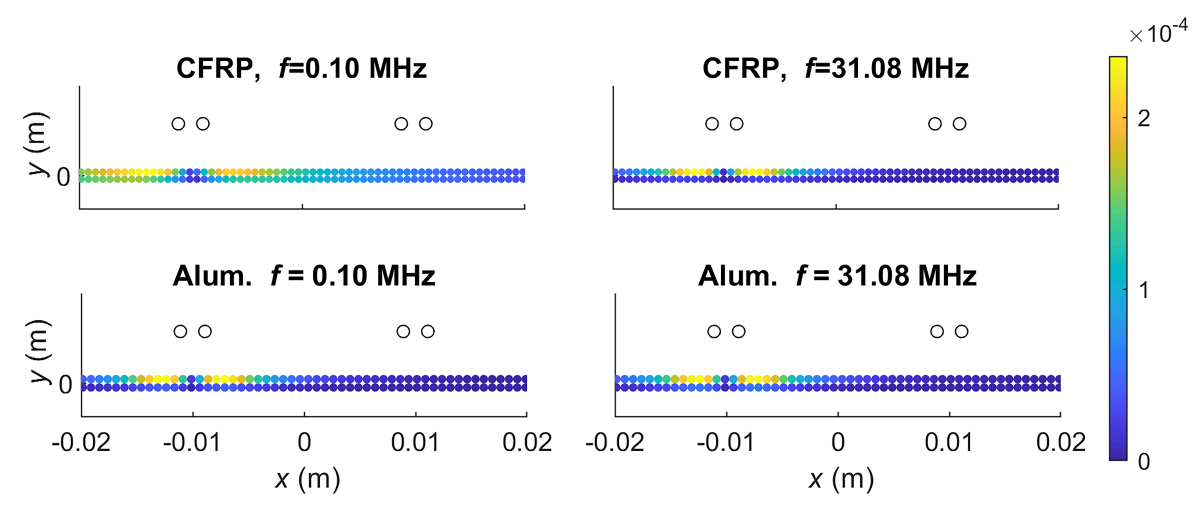

Figure 4.10: Simulated currents (in ampere) at the near-end side of all conductors in the ground plane at 2 different frequencies, for both CFRP and aluminium.

\subsubsection{Double ground plane}

To further illustrate the capabilities of the proposed MTL-DG method, this section shows results of crosstalk between wire pairs between two ground planes. The cross-section is illustrated in Figure 4.2b. Such a configuration can be interesting as a step towards simulation of integrated or embedded wiring, which is of interest to the aviation industry.

Figure 4.11 shows simulated NEXT results for wire pairs between two ground planes, with $h_{1}=h_{2}=4 \mathrm{~mm}$. As reference, the results without nearby ground plane are also shown. Clearly, crosstalk is even further reduced when a second ground plane is placed above the wire pairs. For a single ground plane, 


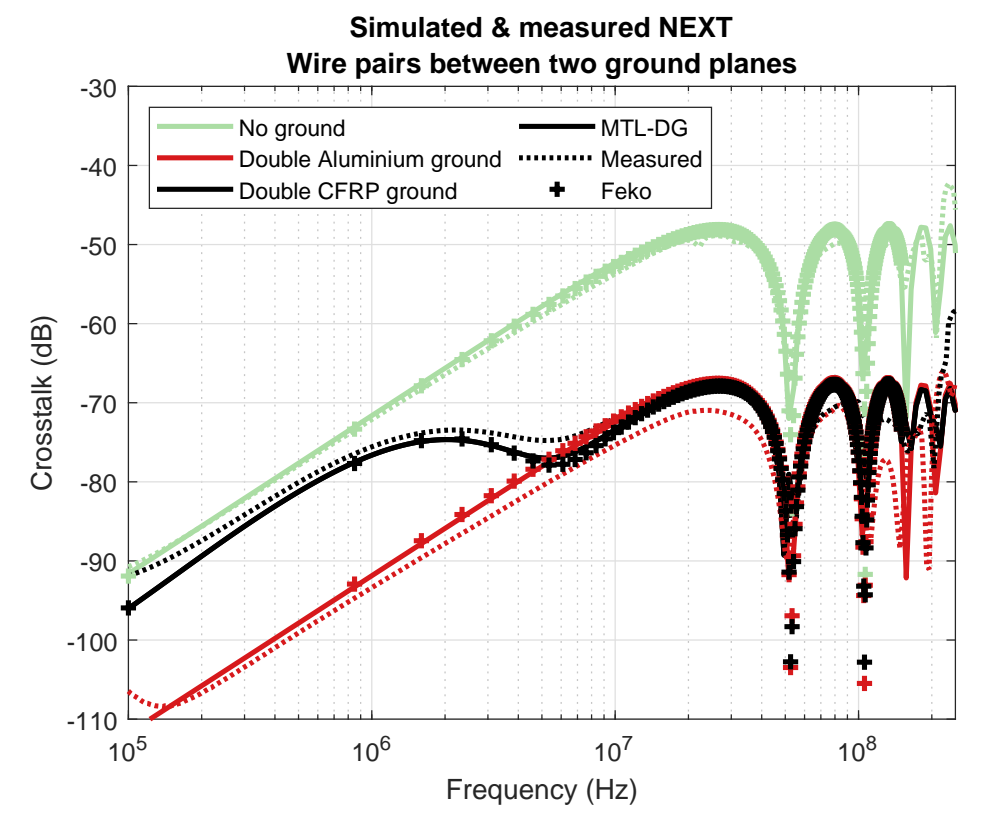

Figure 4.11: Comparison between simulated (MTL-DG - solid lines, Feko - markers) and measured NEXT (dashed lines) between two wire pairs between two ground planes.

the decrease was roughly $8 \mathrm{~dB}$ compared to the case without ground plane. From Figure 4.11 we see that introduction of a second ground plane decreases crosstalk with another $15 \mathrm{~dB}$, for the case under study. Moreover, it can be observed that the general behaviour of crosstalk between CFRP ground planes shows the same tendency as crosstalk above a single CFRP ground plane, but with a different level. Again, for low frequencies the crosstalk is similar to that of wire pairs without nearby ground plane. Figure 4.11 shows that the agreement between measurements and MTL simulations are very good for all three cases. The results of the MTL-DG method and Feko show a perfect match for all three cases. However, for the case of two ground planes, the MTL-DG method requires only 40 seconds to run 200 frequencies on a laptop, while Feko computation times vary for $h=4 \mathrm{~mm}$ to $h=1.1 \mathrm{~mm}$ from 180 to 14.100 seconds, to run a single frequency on 10 cores of our Linux server. This implies a factor 900 - 70,000 simulation time improvement of the MTL-DG method, when compared to Feko.

Finally, figures 4.12 and 4.13 show results of MTL-DG and Feko simulations, and measurements for two wire pairs between two aluminium and two CFRP ground planes. The lower ground plane is kept $3.5 \mathrm{~mm}$ from the wire pairs, while the distance of the upper ground plane is varied. For all distances, MTLDG and Feko again show a perfect match. Also the simulations and measurements coincide very well. Only for the CFRP ground planes slight differences occur, which are most likely caused by uncertainties in the measure- 


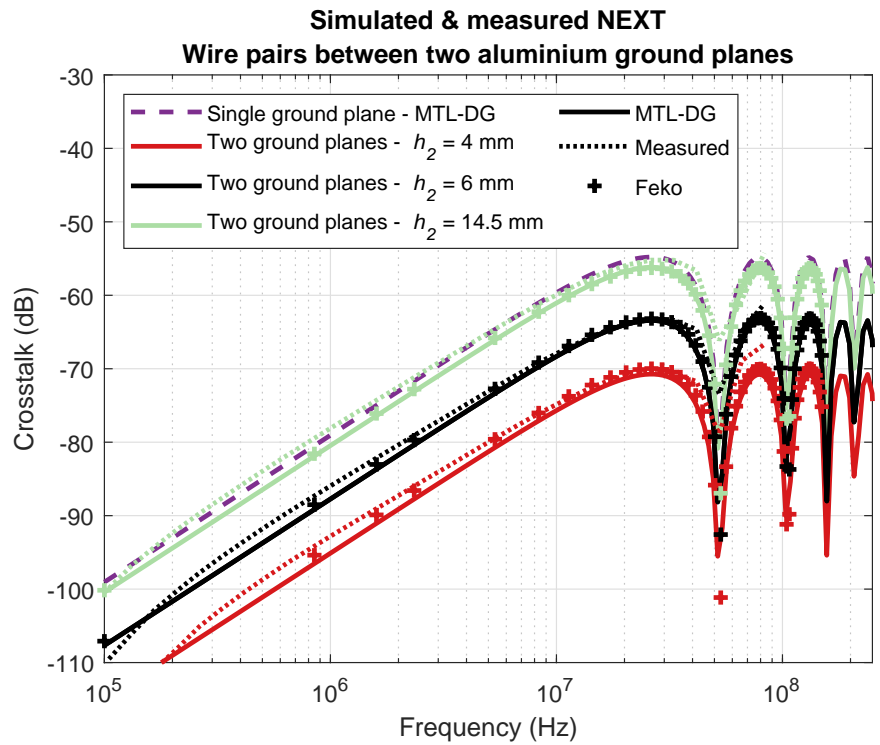

Figure 4.12: MTL-DG (solid lines), Feko (markers) and measured (dotted lines) NEXT for two wire pairs between two aluminium ground planes. The lower ground plane is at 3.5 $\mathrm{mm}$, while the height of the other ground plane is changed. MTL-DG result for single aluminium ground plane is shown as reference (purple dashed line).

Simulated \& measured NEXT

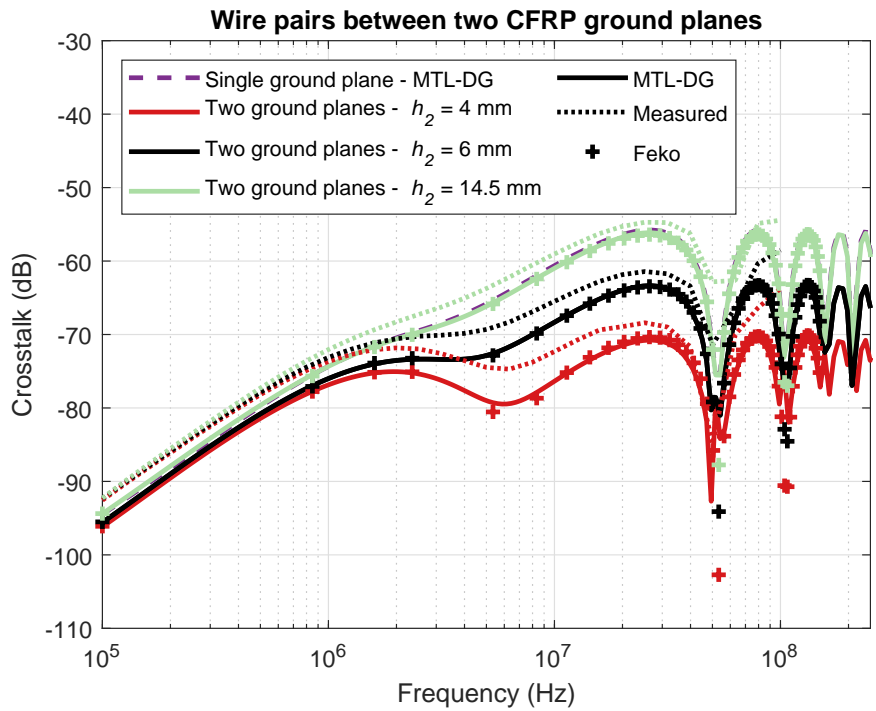

Figure 4.13: MTL-DG (solid lines), Feko (markers) and measured (dotted lines) NEXT for two wire pairs between two CFRP ground planes. The lower ground plane is at $3.5 \mathrm{~mm}$, while the height of the other ground plane is changed. MTL-DG result for single CFRP ground plane is shown as reference (purple dashed line). 
ment setup, or differences between CFRP properties in simulations and measurements. The variations of the height of the second ground plane show that, similar to the case of a single ground plane [22], the effects of the ground plane to the crosstalk behaviour become less when the ground plane is further away. As reference, the simulated result for a single ground plane is also shown in figures 4.12 and 4.13. For both aluminium and CFRP ground planes, this result coincides with the levels of crosstalk obtained for $h_{2}=14.5 \mathrm{~mm}$. This confirms that at this distance the second ground plane has no effect anymore. Bringing the second CFRP ground plane closer has little effect on the low-frequency behaviour, but it does significantly affect the high-frequency crosstalk. Thus, integrating for instance flex PCBs into thermoplastic material that has conducting fibres, such as CFRP, can greatly reduce high-frequency crosstalk levels.

\subsection{Summary}

Replacement of conducting materials in aircraft by less conducting composite materials such as CFRP highlights the need for simulation methods that can quickly assess the effects of lossy ground planes to crosstalk behaviour. This chapter presented two methods that incorporate the effects of ground planes with finite conductivity and thickness into MTL models. As opposed to the previous two chapters these models are not only applicable to electrically short wires, but can be applied under the frequency conditions of MTL theory. The first method makes use of a discretisation of the ground plane by cylindrical conductors which have a diameter equal to the thickness of the ground plane. Well-known analytical expressions can still be used for the inductance and capacitance matrices. When the resistance matrix is adapted to include the analytical resistance of cylindrical wires it is found that the resulting MTL-DG crosstalk simulations coincide very well to measured crosstalk and full-wave Feko simulations, for both metallic ground planes as well as CFRP ground planes.

The second method makes use of a ground impedance matrix. Formulas that are used throughout literature to evaluate the effects of lossy ground to overhead transmission lines are adapted to the case of a CFRP ground plane with finite thickness. Computed crosstalk levels obtained with this MTL-GI method coincide very well with the MTL-DG method, as well as with measured crosstalk and full-wave simulations. Both presented MTL methods show the frequency dependent behaviour of CFRP ground planes that has been observed in an earlier paper [25]. For low frequencies, EM fields penetrate uniformly through the CFRP ground plane, return currents distributed broadly under the wires, and crosstalk levels are similar to that between wire pairs without ground plane. For high frequencies, the fields are unable to penetrate the CFRP further than one skin depth. Therefore, return currents concentrate more at the surface and below the current-driving wire and conse- 
quently crosstalk levels are equal to those for aluminium ground planes. Both thickness and conductivity of the ground plane are important parameters in the switch in frequency behaviour, which appears at the frequency for which the skin depth of the material is in the same order as the thickness of the ground plane.

The MTL-DG method is also applied to the case of two wire pairs between two ground planes. Again, for both aluminium and CFRP ground planes the results of calculations and measurements coincide very well. Crosstalk levels indicate that the presence of a second ground plane close to the wire pairs further reduces the crosstalk, when compared to wire pairs without ground plane and above a single ground plane. For CFRP ground planes this effect is again only observed for the higher frequencies. For low frequencies, the crosstalk is more similar to that of wire pairs without nearby ground plane. Moving one of the two ground planes away from the wire pairs increases highfrequency crosstalk, up to a point where the levels are equal to the case of a single ground plane.

Concluding, the two MTL methods that include lossy grounds presented in this chapter yield simulation results that show a perfect match to full-wave simulations and match measurements well. However, computation times on a simple laptop can be from 900 up to 70,000 times better compared to Feko on a 10 core Linux server. 


\section{5 || Applications of crosstalk models}

A EWIS contains complex cable bundles with uncertainties in various model parameters, such as separation distances and twist rates. Such uncertainties can highly complicate a correct prediction of crosstalk levels. A sensitivity analysis can then be useful to detect the most sensitive parameters. Sensitivity analysis can be performed in various ways. Paul experimentally determined sensitivities of crosstalk with respect to wire positions [56]. Analysing closedform expressions for their level of dependency on different parameters is another alternative, as was done in [23] as well as in Chapter 2. For more complex cable bundles statistical methods provide a structured way to determine the mean crosstalk and the corresponding standard deviation in various configurations. Therefore, such statistical methods are frequently used to perform sensitivity analysis.

In this chapter MTL models are applied to perform sensitivity analysis to two cases:

- Crosstalk between Twisted Wire Pairs (TWPs) inside a bundle shield

- Transfer impedance measurement methods

The sensitivity of a quantity, such as crosstalk or transfer impedance, with respect to any model parameter can be defined by the ratio of standard deviations of output and input. The standard deviation, $\sigma$, should be normalised by the corresponding mean value, $\mu$, yielding a quantity that is called the coefficient of variation $c_{v}$ :

$$
c_{v}=\frac{\sigma}{\mu}
$$

Then the sensitivity of for instance crosstalk with respect to an input parameter is given by the ratio of the estimated $c_{v, \gamma}$ for crosstalk and the assumed $c_{v, i}$ in the input parameter:

$$
S=\frac{c_{v, \gamma}}{c_{V, i}}
$$

To determine the sensitivity with respect to various model parameters a stochastic distribution with certain mean and standard deviation is assumed 
for each input parameter that is under evaluation. Statistical methods can then be applied to estimate the resulting mean and standard deviation in the output. Multiple run algorithms such as the conventional Monte Carlo (MC) method are a well-known way of determining statistical moments of a stochastic process. In [11] the authors use MC simulations to investigate the influence of non-uniform twisting and incomplete final twists to radiated susceptibility. For cable bundles with non-uniformities such as twisting and meandering of cables, MC computation times can become severe. Since MC requires a large number of runs, other statistical methods are preferred. Many efficient alternatives have been presented in literature [15, 17, 18]. In [13,57], Fei introduced the Stochastic Reduced Order Model (SROM) in an EMC context. This statistical method is non-intrusive and quite straight forward to implement and in $[13,57]$ it was used to investigate the effect to crosstalk between two single wires above a ground plane when height, separation distance, wire radius and the termination impedance were changed. Similarly, in [58] it was used to determine the impact of different parameters of an incident plane wave coupling into a transmission line. In this chapter, the SROM method is used for a sensitivity analysis for crosstalk in a bundle of twisted wire pairs with respect to parameters such as twist rate and cable meandering, as well as the influence of dielectric permittivity and termination mismatches in transfer impedance measurement setups. Moreover, for the simulation of twisted pairs, the method to make the MTL simulations more efficient mentioned in Section 2.3 is applied.

The SROM method is used to estimate the mean and standard deviation of the output parameter, for instance NEXT. In comparison to the MC method, the SROM method is a multiple run method that contains a limited set of sample values for the uncertain input parameter (compare: order of 10 (SROM) versus order of $10^{5}(\mathrm{MC})$ for one uncertain parameter). These samples are chosen in such a way that the SROM accurately describes the probability distribution of the input variable. An optimal SROM can be obtained by using the pattern classification method (see [13]). Output of this algorithm is a set of $m$ realisations of the model parameter that forms the $\operatorname{SROM}, \hat{\mathbf{x}}=\left(\hat{x}_{1}, \ldots, \hat{x}_{m}\right)$, including corresponding probabilities for each sample $\mathbf{p}=\left(p_{1}, \ldots, p_{m}\right)$. Figure 5.1 illustrates the selection of such an optimal set of 25 SROM realisations when two stochastic parameters would be assumed (for this example separation distance and height above ground were chosen, with $\mu$ equal to $6 \mathrm{~mm}$ and $10 \mathrm{~cm}$ and $\sigma$ equal to $0.6 \mathrm{~mm}$ and $1 \mathrm{~mm}$, respectively). The pattern classification is based on Voronoi regions, which are also shown in Figure 5.1. The SROM samples have the property that their statistics are similar to the 20.000 MC samples that are shown. For further details, we refer to [13].

Since for sensitivity analysis we consider one parameter at a time, $\hat{x}_{i}$ represents a scalar sample. In this chapter, we choose a number of $m=25$ samples. This number is higher than was concluded for a single variable uncertainty in [13]. However, sensitivity of crosstalk with respect to for instance twist rate (which will be computed in Section 5.1) can be very high and therefore we use 


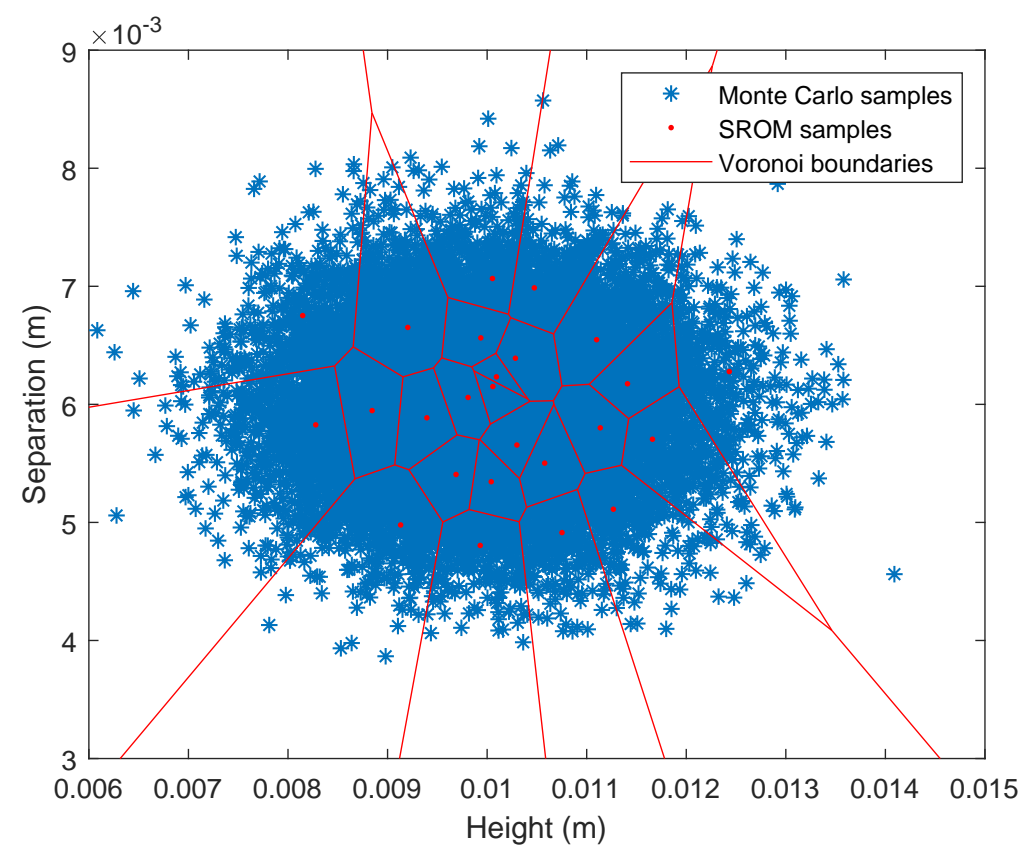

Figure 5.1: Example of the selection of 25 optimal SROM samples (red dots) from 20.000 MC samples, for the case of two stochastic variables (height above ground and wire pair separation). The Voronoi regions that are used in the pattern classification are also shown.

more samples to increase the accuracy of the SROM results. Still, compared to the MC method, which needs in the order of 100 thousand runs for convergence, a huge decrease in computation time can be achieved.

To determine the sensitivity of for instance crosstalk with respect to an input parameter, for each realisation $k$ of the SROM the corresponding nearend crosstalk $\gamma_{N E, k}$ is computed. Statistical moments of crosstalk are then estimated by:

$$
\begin{aligned}
& \hat{\mu}_{\gamma}=\sum_{k=1}^{m} p_{k} \gamma_{N E, k} \\
& \hat{\sigma}_{\gamma}=\sum_{k=1}^{m} p_{k}\left(\gamma_{N E, k}-\hat{\mu}_{\gamma}\right)^{2} .
\end{aligned}
$$

For Section 5.2, the procedure will be entirely equal, however the output under investigation will be transfer impedance instead of crosstalk.

A useful way of displaying sensitivity results is shown in Figure 5.2. The dashed lines indicate various zones of sensitivity. Below $\mathrm{OdB}$, the sensitivity is such that the variability in the output is less than in the input. The further below $\mathrm{O} \mathrm{dB}$, the more we can neglect the uncertainty in this model parameter. 


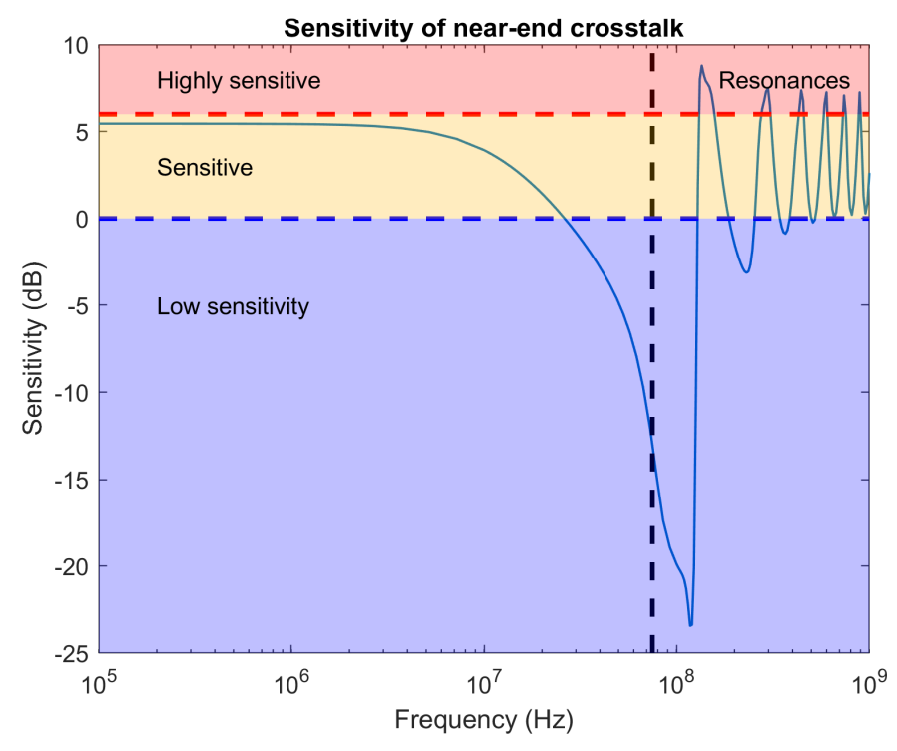

Figure 5.2: Layout for sensitivity plots.

In the orange area, the relation between variability in input and output ranges from linear $(\mathrm{O} d \mathrm{~B})$ to quadratic $(6 \mathrm{~dB})$. Above this, the sensitivity becomes really high. In the low-frequency area we can also distinguish between inductive and capacitive crosstalk by creating cases with low and high-impedance terminations. Above the dashed black line frequencies become high enough for resonances to occur, which can also be reflected into sensitivity results. For instance when the sensitivity with respect to permittivity is investigated, a slight change in permittivity will shift the resonance frequencies slightly. At that precise frequency, the crosstalk value can change drastically by that change of permittivity, yielding peaks in the sensitivity analysis while in general the effect to the entire crosstalk curve is relatively small. Therefore, in this high-frequency area one has to be cautious while interpreting the sensitivity values.

In Section 5.1, which is based on [35] the above methodology is used to investigate the sensitivities of crosstalk between twisted wire pairs in a shielded bundle. Input parameters under investigation include intra-pair separation distance and twist rates. In Section 5.2, which is based on $[59,60]$ MTL models are developed for transfer impedance measurement methods. The sensitivity of line injection and triaxial methods are investigated with respect to dielectric permittivity and possible mismatches in the terminations. Section 5.3 summarizes the findings in this chapter. 


\subsection{Twisted pairs in a bundle}

The authors of [13] applied the SROM method to crosstalk in the simple configuration of two wires above a ground plane. In this section, the SROM method is applied to two cable bundles that contain two and seven twisted wire pairs, respectively. In two crosstalk configurations concerning these bundles, sensitivity with respect to inter-pair and intra-pair separation distance is investigated. Moreover, twist rate sensitivity is discussed for twisted pairs with equal and unequal twist rates. Thereafter, in the second bundle both the bundle twist and the meandering of individual cables are included to investigate their effects on various parameter sensitivities. Realisations of meandering cables are obtained by the Random Displacement Spline Interpolation (RDSI) method [9]. The resulting non-uniform transmission line equations are solved by the UCS method.

\subsubsection{Cable modelling}

Consider the two cable bundles of which the cross-sections are shown in Figure 5.3. Both bundles are enclosed by a bundle shield and contain two and seven TWPs, respectively. For both cases, the radii of the shield $r_{s}$ and wires $r_{w}$ are $6.8 \mathrm{~mm}$ and $0.4 \mathrm{~mm}$, respectively. Moreover, the wire pairs are all separated $3.4 \mathrm{~mm}$ from the centre of the bundle, i.e. $d_{i}=3.4 \mathrm{~mm}$ (except for the centre pair in Figure 5.3b). The intra-pair separation distance $a$ between the individual wires of a single pair is $1.1 \mathrm{~mm}$. The length of the cable bundles $\ell$ is $1 \mathrm{~m}$ and the terminations of all individual wire pairs are as in Figure 2.4. Here the differential-mode resistance is either $50 \Omega$ or $1000 \Omega$ to define a low and high-impedance case, respectively.

The voltages and currents in the cable bundles shown in Figure 5.3 will be solved by the MTL equations. The cable bundles in Figure 5.3 are non-uniform along their length, which is caused by the combination of twisted wire pairs, bundle twisting and meandering cables. Therefore, the UCS as extension to Paul's MTL theory is used to simulate the crosstalk behaviour in the bundles, as it has also been applied in [7]. However, as introduced in Section 2.3, to obtain a significant speed-up of the sensitivity computations the low-frequency formulation in equation (2.10) will be used to compute the solution of each cascaded section.

The per-unit-length inductance matrix for each cascaded section can be computed by [5]:

$$
\begin{aligned}
& l_{i i}=\frac{\mu_{0}}{2 \pi} \ln \left(\frac{r_{s}^{2}-d_{i}^{2}}{r_{s} r_{w}}\right) \\
& \iota_{i j}=\frac{\mu_{0}}{4 \pi} \ln \left(\frac{1}{r_{s}} \sqrt{\frac{\left(d_{i} d_{j}\right)^{2}+r_{s}^{4}-2 d_{i} d_{j} r_{s}^{2} \cos \theta_{i j}}{d_{i}^{2}+d_{j}^{2}-2 d_{i} d_{j} \cos \theta_{i j}}}\right) .
\end{aligned}
$$




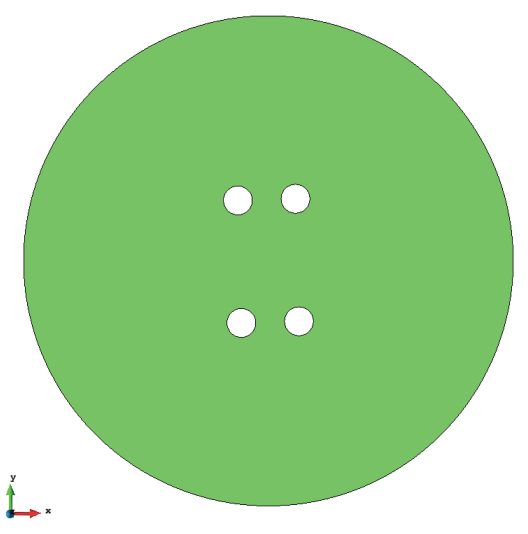

(a) A bundle with 2 twisted pairs.

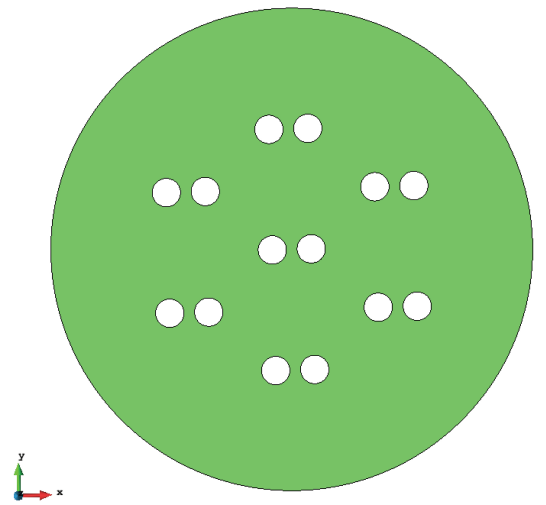

(b) A bundle with 7 twisted pairs.

Figure 5.3: Cross-sections of the two bundles to which sensitvity analysis is performed.

Here $d_{i}$ is the separation distance from conductor $i$ to the centre of the bundle and $\theta_{i j}$ the angle between conductors $i$ and $j$ in the bundle. It is assumed that the bundle contains a homogeneous medium, by which the capacitance matrix follows from equation (2.27). This implies that the insulation of wires can only be taken into account by an effective, homogeneous relative permittivity. Modelling the actual effects of non-homogeneous media like insulation requires the computationally expensive numerical estimation of p.u.l. parameters. However, the permittivity will only affect the average crosstalk and corresponding resonance frequencies, but not the sensitivity with respect to other model parameters. Therefore, in this section we choose the medium inside the bundle to be free space.

The meandering of the cables along the length of the bundle is included via the RDSI algorithm [9]. This is applied to each twisted wire pair. To this extent, the start and end positions of all TWP centres are fixed in accordance with Figure 5.3. Along its length, the cable is subdivided into 15 segments, to which a random displacement is added in the two orthogonal cross-sectional directions. These displacements are normally distributed with mean zero and standard deviation taken equal to $d_{i} / 2$. The segments are subdivided into smaller sub-segments on which spline interpolation is performed. This yields for TWP $i$ an $x$ and $y$ coordinate on each sub-segment, which are denoted by $x_{\text {twpi,s }}\left(z_{k}\right)$ and $y_{\text {twpi,s }}\left(z_{k}\right)$ and where the longitudinal position along the TL is $z_{k}$. The length of the sub-segments is determined by the minimum of either a tenth of a wavelength or the amount of cascaded sections that are needed to accurately model one twist. To the interpolated coordinates on these sub- 
segments the bundle twist is introduced by the following addition:

$$
\begin{aligned}
& x_{\text {twpi }}\left(z_{k}\right)=x_{\text {twpi,s }}\left(z_{k}\right)+x_{B, i}\left(z_{k}\right) \\
& y_{\text {twpi }}\left(z_{k}\right)=y_{\text {twpi,s }}\left(z_{k}\right)+y_{B, i}\left(z_{k}\right),
\end{aligned}
$$

in which:

$$
\begin{aligned}
& x_{B, i}\left(z_{k}\right)=d_{t w p i} \sin \left(\theta_{B}\left(z_{k}\right)\right) \\
& y_{B, i}\left(z_{k}\right)=d_{t w p i} \cos \left(\theta_{B}\left(z_{k}\right)\right)
\end{aligned} \text { with } \quad \theta_{B}\left(z_{k}\right)=\theta_{0}+\frac{2 \pi z_{k} N_{B T w}}{\ell} .
$$

Here $x_{B, i}$ and $y_{B, i}$ describe the deviations that are caused by the bundle twist. $N_{B T w}$ is the total amount of twists in the bundle. Usually, the length of a full bundle twist is equal to the length of the bundle divided by its diameter. Therefore, we take $N_{B T w}$ equal to the first integer number larger than the bundle length divided by this twist length. In our case, this equals 8 bundle twists. Thereby, $x_{t w p i}\left(z_{k}\right)$ and $y_{t w p i}\left(z_{k}\right)$ control the positions of the centres of twisted wire pair $i$ along the transmission line, including meandering and bundle twist. The positions of individual conductors 1 and 2 of such a twisted pair are obtained by adding coordinates of a helix form to each of the uniform sections:

$$
\begin{aligned}
& x_{h, 1}\left(z_{k}\right)=+(a / 2) \cos \left(\phi_{k}\right) \\
& y_{h, 1}\left(z_{k}\right)=+(a / 2) \sin \left(\phi_{k}\right) \\
& x_{h, 2}\left(z_{k}\right)=-(a / 2) \cos \left(\phi_{k}\right) \\
& y_{h, 2}\left(z_{k}\right)=-(a / 2) \sin \left(\phi_{k}\right)
\end{aligned}
$$

With $\alpha_{t w p i}$ the starting angle of each twisted wire pair at $z=0$ can be controlled. In this section, the starting angles are as shown in Figure 5.3, yielding $\alpha=0$. The positions of each conductor along the length of the line can now be computed:

$$
\begin{aligned}
& x_{\text {twpi,p }}\left(z_{k}\right)=x_{\text {twpi }}\left(z_{k}\right)+x_{h, p}\left(z_{k}\right) \\
& y_{\text {twpi,p }}\left(z_{k}\right)=y_{\text {twpi }}\left(z_{k}\right)+y_{h, p}\left(z_{k}\right) .
\end{aligned}
$$

In this, the subscript $p$ represents the conductor of a specific wire pairs, therefore it is either 1 or 2 . From this, the corresponding angles $\theta_{i j}$ and separation distances $d_{i}$ for all the individual conductors inside the bundle shield can be computed, from which the inductance and capacitance can be computed by (5.4). The above mentioned cascaded MTL theory is then used to solve the termination currents and voltages on each conductor, from which by equation (2.3) crosstalk is computed.

\subsubsection{Sensitivity simulation results}

This section describes the results of sensitivity analysis for the following two situations: 
1. Case 1 - Crosstalk between the two twisted wire pairs in bundle 1 .

2. Case 2 - Crosstalk between the centre and top twisted wire pairs in bundle 2.

In the following, firstly a comparison will be made between the sensitivity in these cases with respect to parameters like inter and intra-pair separation distance, as well as the twist rate. Here both bundle twist and cable meandering are not taken into account. The parameters under investigation are assumed to follow a normal distribution, of which mean and standard deviation will be given. Secondly, the bundle twist is included to observe its influence to parameter sensitivities. Finally, also the cable meandering is included.

In aircraft industry the application of twisted wire pairs with equal twist rate is very realistic. However, it is known that combinations of twisted pairs with a difference in twist rate are preferred from a crosstalk perspective. Therefore, sensitivities and average crosstalk for both cross-sectional parameters and twist rates are discussed for situations concerning twisted pairs with equal and unequal twist rates.

\section{No bundle twist and no meandering}

Consider the cable bundles introduced in Figure 5.3 and assume that the only non-uniformity of the cable along its length is the twisting of wire pairs. Bundle twists and cable meandering are not taken into account in this section.

Figure 5.4 shows the results of sensitivity analysis with respect to the interpair separation distance for case 1 and case 2 . For case 1 only the low-impedance case is shown, for which inductive coupling is dominant. Distinction is made between the case where both pairs have the same twist rate of 51 twists per metre (tpm), and where the two pairs have different twist rates of $51 \mathrm{tpm}$ and $71 \mathrm{tpm}$. For these results the computed $\mathrm{MC}$ sensitivities are also shown. These are obtained by simulations of 10 thousand realisations of the separation distance. For case 1 the separation distance is assumed to be normally distributed with a mean of $6.8 \mathrm{~mm}$ and a standard deviation of $0.68 \mathrm{~mm}$, while for case 2 the mean and standard deviation are $3.4 \mathrm{~mm}$ and $0.34 \mathrm{~mm}$, respectively (see also Figure 5.3b - case 2 considers crosstalk between centre and top TWP).

The MC simulations validate the SROM results, since they show a perfect match for the separation distance sensitivity for both equal twist rates and different twist rates, as is shown in Figure 5.4. Moreover, the results show that there can be large differences in sensitivities when the twist rate of one twisted pair is changed. The difference in sensitivity with respect to the separation distance for the case of equal twist rate is roughly $14 \mathrm{~dB}$ when compared to unequal twist rate. This change in twist rate also greatly decreases the average near-end crosstalk, as will be concluded from Figure 5.6.

Figure 5.4 also shows a sensitivity result for case 2 . The average separation distance is here $3.4 \mathrm{~mm}$, which is smaller than in case 1. The logarith- 


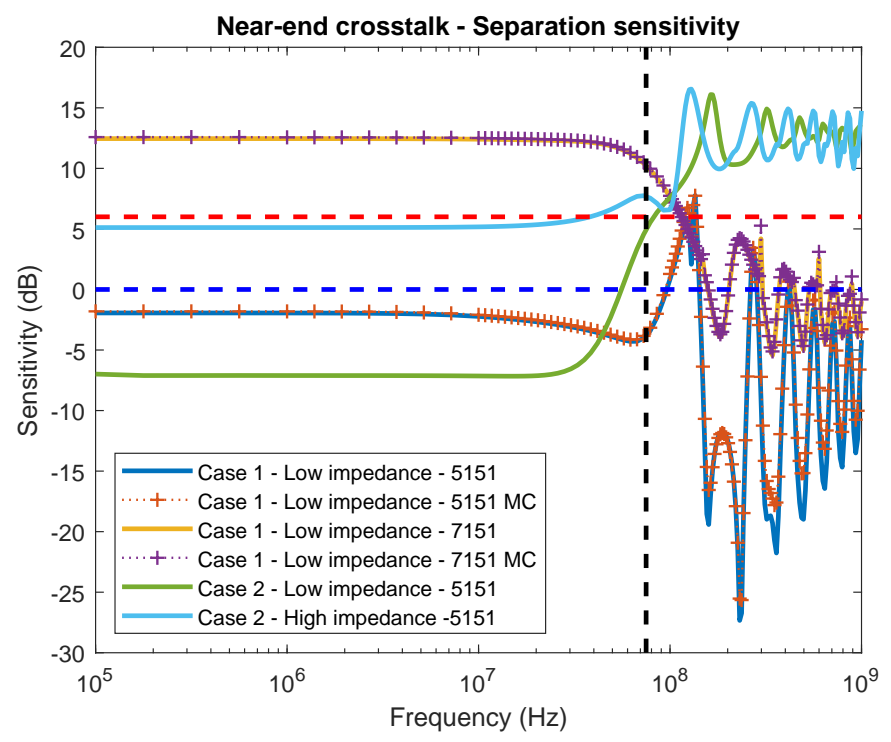

Figure 5.4: Sensitivity with respect to inter-pair separation. Comparison between sensitivities when both TWPs have twist rate equal to 51 (blue) and when twist rates are 71 and 51 (yellow). MC results are shown for validation (red and purple with '+' markers). Sensitivities for high and low-impedance terminations in case 2 are shown (light-blue and green). Horizontal and dashed lines indicate the different regions explained in Figure 5.2.

mic terms in the inductance matrix then cause that this lower separation distance also implies a lower sensitivity on separation distance. As is shown by Figure 5.4 sensitivities differ for inductive and capacitive crosstalk. In the highimpedance case the capacitive crosstalk dominates inductive crosstalk for the frequencies below the resonance area. This shows that capacitive crosstalk is roughly $12 \mathrm{~dB}$ more sensitive to separation distance than inductive crosstalk.

Apart from the cross-sectional parameters, sensitivity analysis can also be performed with respect to twist rates of twisted wire pairs. Figure 5.5 shows the results of such sensitivity analysis by the use of SROM and MC methods. In this case, 100 thousand MC simulations were used. Results of the lowimpedance case are shown, since the twisting is mostly used to reduce inductive crosstalk. Both results for the case of equal and unequal twist rates are given. In Figure 5.6 the corresponding mean values for near-end crosstalk are given. In the statistical simulations the victim pair is assumed to have a twist rate that is normally distributed around $51 \mathrm{tpm}$ with a standard deviation of $5.1 \mathrm{tpm}$. Therefore, the twist rate can be a non-integer, yielding incomplete terminal twists. The culprit transmission line has a fixed twist rate of either 51 or 71 twists per metre. If both twisted pairs were to be modelled with complete terminal twists, a discrete distribution such as the binomial distribution with parameters $n=104$ and $p=51 / 104$ could form an alternative. Such results 
are not shown here.

SROM and MC sensitivity results coincide quite well in the entire frequency range when the twist rates are equal. In case the twist rates are unequal there is a difference in the results. Moreover, for various SROM simulations the sensitivity shows variability of 1 or $2 \mathrm{~dB}$. Actually, even for the $10^{5} \mathrm{MC}$ simulations sensitivity results still show some variations. However, the average crosstalk has converged and $\mathrm{MC}$ and SROM results match perfectly for both cases (see Figure 5.6). Apparently, these statistical estimations of the standard deviation for twist rate uncertainties are less accurate for the case with unequal twist rates in victim and culprit TWPs.

Both SROM and $M C$ results indicate that the average crosstalk decreases by roughly $25 \mathrm{~dB}$ when the twist rate of the culprit pair is changed from 51 to $71 \mathrm{tpm}$. Actually, if incomplete terminal twists would be avoided this decrease can even be larger. Also the sensitivity of crosstalk with respect to the twist rate decreases when unequal twist rates are used. However, in general crosstalk values are very sensitive with respect to these twist rates.

\subsubsection{Influence of bundle twist and meandering cables}

In this section, the bundle twist is included in the model to investigate its influence to sensitivity results. Subsequently, cable meandering is also taken into account. The meandering of cables along the length of the bundle introduces new stochasticity, since the deviations from the connector positions are normally distributed. In principle, statistical simulations are therefore also applicable to cable meandering, yielding an average crosstalk for the combined uncertainty in cable meandering and another model parameter. However, to determine whether there is any effect of cable meandering at all, in this section we stick to repetition of the SROM sensitivity analysis for a certain model parameter for a few realisations of meandering cables. Each realisation results in an average crosstalk and sensitivity for that specific model parameter. Sensitivities of various realisations are then compared to investigate possible variations caused by the meandering. Average values for model parameters are equal to those in the previous sections, and in this case we discuss lowimpedance terminations. The intra-pair separation distance is assumed to be normally distributed with a mean of $1.1 \mathrm{~mm}$ and a standard deviation of $0.11 \mathrm{~mm}$.

Figure 5.7 shows results of sensitivity analysis for case 2 with respect to the inter and intra-pair separation distance. In both cases, the twisted pairs have equal twist rate $(51 \mathrm{tpm})$. The results in this section are all given in a similar way, where the solid line represents sensitivity without bundle twist and cable meandering, the dotted line with ' + ' markers includes bundle twist and the dotted line with 'o' markers includes both bundle twist and cable meandering. The results in Figure 5.7 show that bundle twist and cable meandering barely influence the sensitivity with respect to separation distance. For this case, only one realisation of cable meandering is shown, since other realisa- 


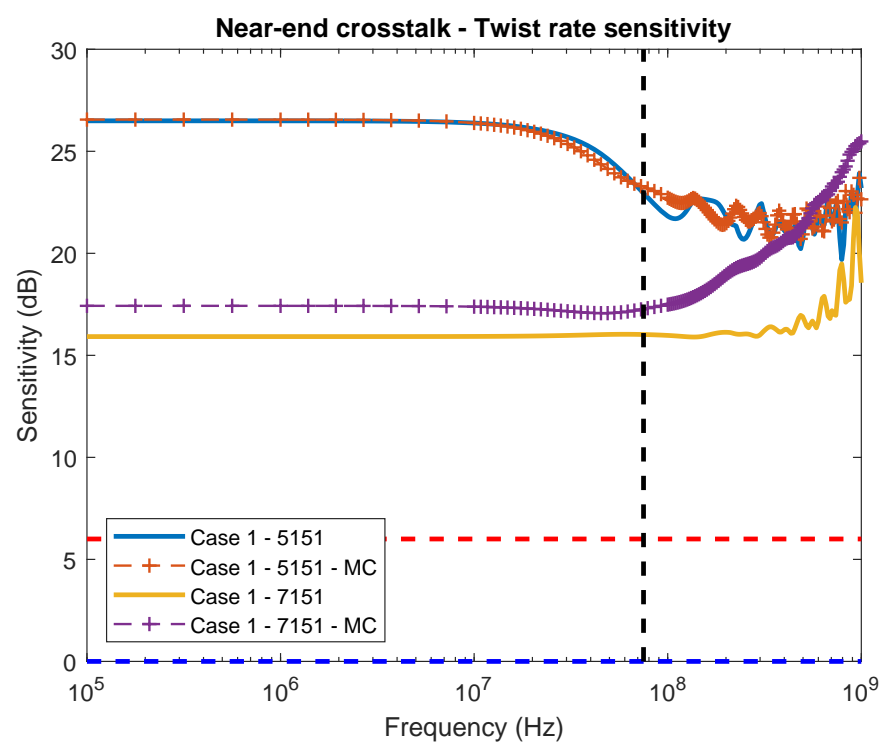

Figure 5.5: Sensitivity with respect to twist rate. Comparison between sensitivities when both TWPs have twist rate equal to 51 (blue), and when twist rates are 71 and 51 (yellow). MC results are shown for validation (red and purple with '+' markers). Horizontal and dashed lines indicate the different regions explained in Figure 5.2.

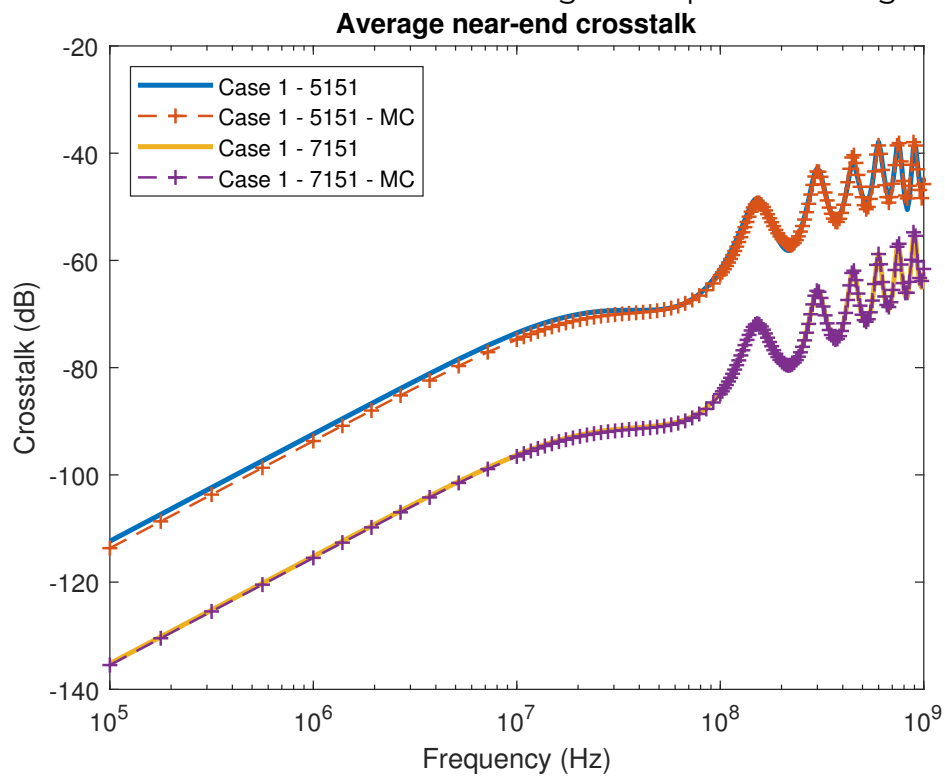

Figure 5.6: Average crosstalk with uncertain victim twist rate. Results are shown when both TWPs have a twist rate equal to 51 (blue), and when the twist rates are 71 and 51 (yellow). As validation, Monte Carlo results are also shown (red and purple with '+' markers). 


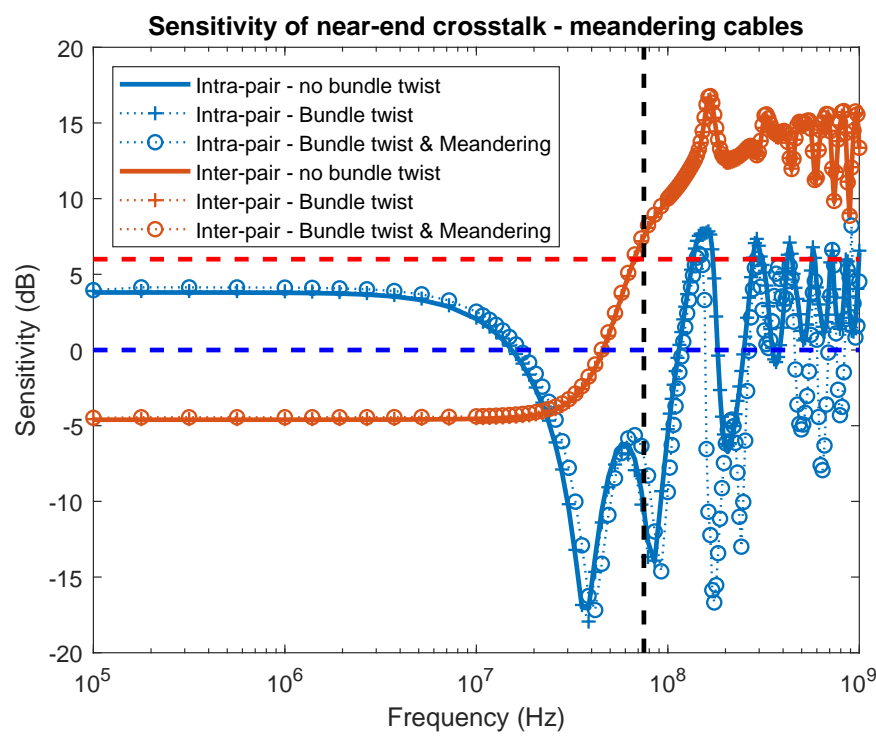

Figure 5.7: Sensitivity of NEXT for case 2 with respect to the intra-pair (blue) and interpair (red) separation. Solid lines represent the case of a bundle with straight cables, i.e. no bundle twist and no meandering. For the dotted lines with '+'-signs bundle twist is included, and for the 'o'-marked lines also cable meandering is included. Horizontal and dashed lines indicate the different regions explained in Figure 5.2.

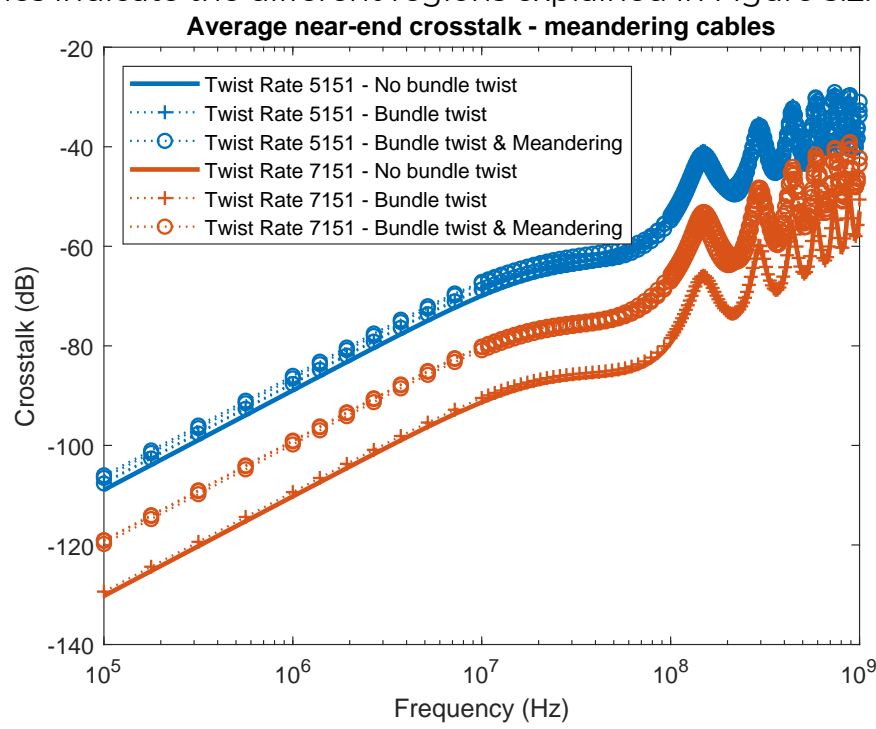

Figure 5.8: Average NEXT in case 2 for twist rate uncertainty for 51 tpm versus 51 tpm (blue) and $71 \mathrm{tpm}$ versus $51 \mathrm{tpm}$ (red). Solid lines represent the case of a bundle with straight cables, dotted '+'-marked lines include bundle twist and 'o'-marked lines also include cable meandering. 
tions yield the same result. In the high-frequency area, only the sensitivity with respect to intra-pair separation deviates slightly when the cable meandering is introduced. However, for all other sensitivities the bundle twist and cable meandering could be neglected.

Since for twist rate uncertainties SROM sensitivity values showed to be slightly unstable, Figure 5.8 shows corresponding average crosstalk results when the bundle twist or cable meandering is included. Multiple realisations of cable meandering are shown, which lead to equal results for the case of equal twisting. For unequal twisting the bundle twist has no influence on the average crosstalk, while cable meandering increases the results with roughly 10 $\mathrm{dB}$. However, various realisations of meandering cables yield results that are nearly equal along the entire frequency range.

\subsection{Transfer impedance}

Cable shields or screens, which were already introduced in Chapter 3, can be used to protect cabling from both crosstalk and external effects, such as High Intensity Radiated Fields (HIRF) and lightning. With that purpose braided shields are used in aviation industry (see Figure 5.9). These shields are composed of strands with small copper wires that are woven together into a braided shield around for instance shielded twisted pairs or entire cable bundles. Adding extra layers of shielding can improve EMC, but will simultaneously increase the on-board weight significantly. For optimisation of shielding from an EMC perspective it is of high importance to cable manufacturers to obtain a measure for the quality of shields.

The performance of braided shields is expressed in transfer impedance $Z_{T}$, which is a measure for the coupling between the domains internal and external to the shield. It is defined as the ratio of induced voltage on the inside of the shield over the current flowing on its outer surface. This concept was initially introduced by Schelkunoff [62] and the formal definition is given by:

$$
Z_{T}=\frac{1}{I_{0}} \frac{\partial V}{\partial Z} .
$$

Here $I_{0}$ is the current flowing on the outside of the shield and $\partial V / \partial z$ is the voltage per unit length induced on the inside of the shield. Thus, following this definition the voltage drop inside the cable shield over an infinitesimal piece of cable is divided by the current outside the shield evaluated at the same position along a transmission line.

Transfer impedance is a theoretical and intrinsic parameter whose value, given in $\Omega / m$ and defined over an infinitesimally small piece of the shield, depends only on the geometry and materials of the braid. It allows estimating the electromagnetic effect in the wires inside the cable produced by an external field or, reciprocally, the radiation leaked from inside the cable to the environment. A low $Z_{T}$ indicates little coupling between interior and exterior 


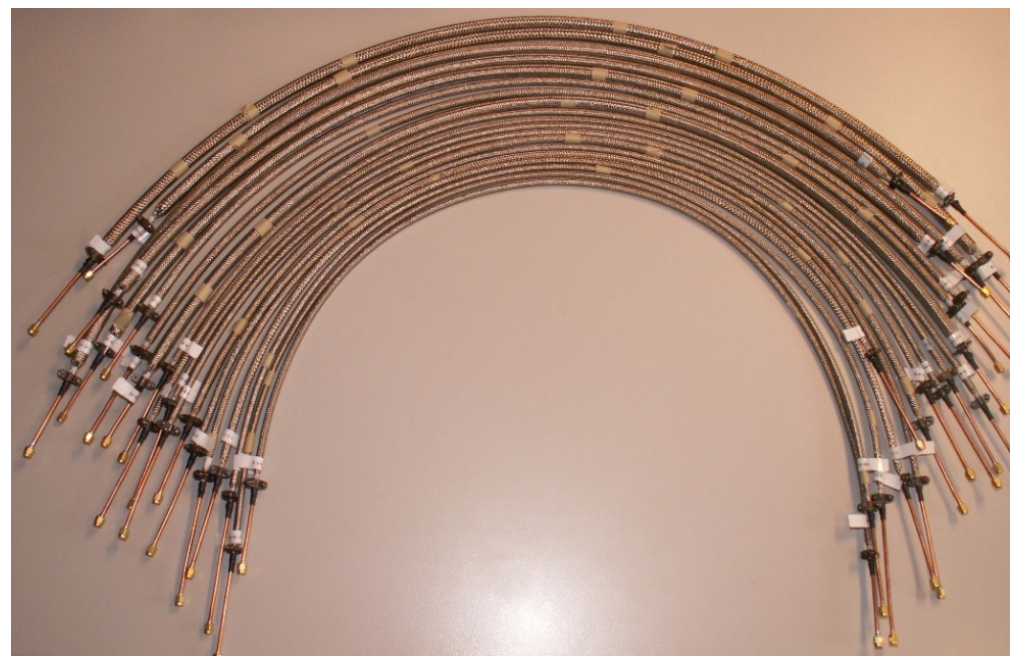

Figure 5.9: Photograph of a variety of manufactured braided shield samples. Transfer impedance of these shielded cables has been analysed in $[60,61]$.

of the shield, and therefore a good shielding against interfering electromagnetic fields. Several analytical models have been derived for the prediction of transfer impedance based on geometrical parameters of the shield [39,63-65]. Since these analytical models are only defined for an infinitesimal small piece of cable screen they do note take into account effects of finite cable length. In these models, for the lower frequency region the transfer impedance is governed by the DC resistance of the braid and the diffusion of waves through the cylindrical braid. For high frequencies the imaginary part of the transfer impedance dominates, comprising a superposition of hole, braid and skin inductance. Each of the analytical models differs in the calculation of hole and braid inductance.

In a transfer impedance measurement of a shielded cable a sample with fixed and finite length is used. In such a case the measured transfer impedance can be regarded as an integral over the length of the line of equation (5.9), resulting in a voltage drop over the length of this cable divided by the corresponding shield currents. In practice, this can be measured in a variety of setups, such as the line injection and triaxial methods [66-68]. In such measurement setups a source is connected to one side of the injection circuit (depending on the measurement setup, this can be either the internal or external circuit), and the resulting voltage drop on the near or far-end side of the other circuit is measured. Indeed, this implies that the integrated effects of (5.9) over the transmission line are measured, leading to the fact that in the measurement of transfer impedance long line effects can be observed that are not part of the definition of transfer impedance. Therefore, in general the obtained measured transfer impedance is only similar to analytical models up 
to a certain frequency [66-73].

Previous literature discusses the influence of propagation effects to measured transfer impedance in high frequency regions [66, 69-73]. Standards specify the frequency validity of results in different measured setups [66-69]. Démoulin analytically derives frequency expressions explaining propagation phenomena for triaxial and line injection methods [70-72]. For these derivations the injection circuit is solved first, after which equivalent distributed sources ensure a one-way coupling of injected currents into the test sample [73]. Finally, Démoulin illustrates the influence of different propagation effects to transfer impedance measurements by some example measurements. The current section of this thesis, which is based on $[59,60]$, introduces a different, MTL approach to simulate transfer impedance measurement setups. This is a generic approach that can be used to perform similar analysis to the propagation effects combined with the finite length and mismatches, and is quickly adapted to various measurement methods. Moreover, it is applied to perform sensitivity analysis with respect to parameters that control mismatches in the terminations of the setups, as well as dielectric permittivity.

The MTL model for these transfer impedance measurement setups uses the addition of shielding to the MTL equations as described in Chapter 5 and $[37,74]$. In this section, the method is used to derive an MTL model for both the line injection and triaxial measurement setup, both extensively described in many standards, among which [66-68]. Moreover, the low-frequency methods previously used in chapters 2 and 3 are applied to obtain transition frequencies similar to $[66,69]$. The developed MTL model is applied to a coax with line injection to analyse simulated transfer impedance measurement results. Distinctions are readily made between transmission lines with perfect terminations and those with mismatches, as well as coaxes with and without dielectrics. These distinctions clarify the origin of certain resonances and transmission line effects to the measured transfer impedance. Based on the method in the beginning of this chapter, also sensitivities of transfer impedance measurements with respect to mismatches and permittivity will be analysed.

Section 5.2.1 introduces the models for the two transfer impedance measurement methods. Section 5.2.2 will give some closed-form expressions for the induced voltage in the coax with line injection, along with important transition frequencies. Finally, Section 5.2.3 will give the results of sensitivity analyses.

\subsubsection{Transmission line model}

\section{Line injection method}

A model for the test section of the line injection method is illustrated in Figure 5.10. It comprises a coax under test and an injection wire separated by a distance $d$. This cable configuration concerns three conductors, of which the 


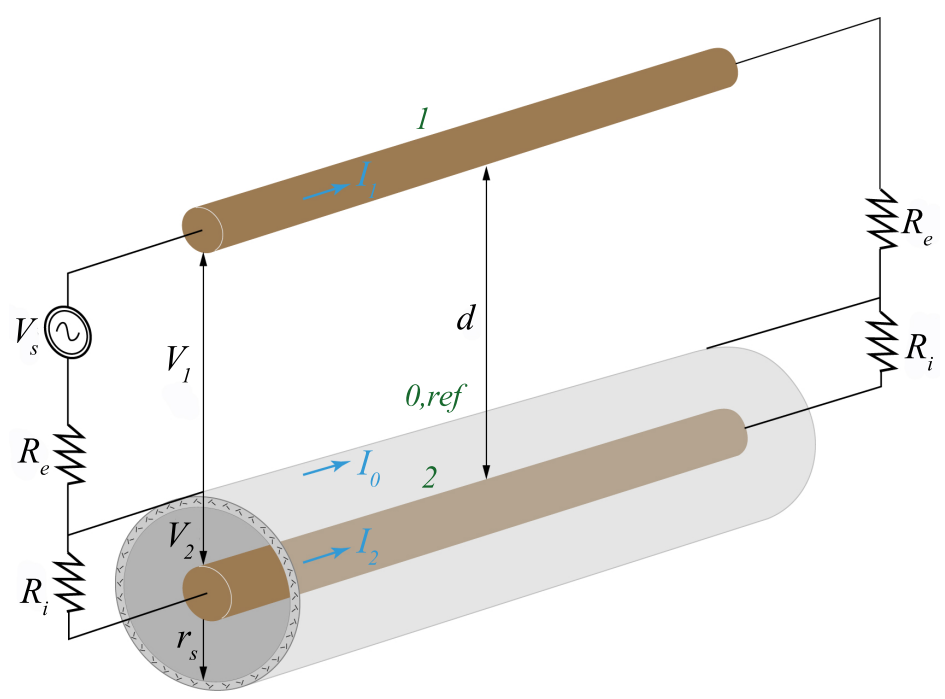

Figure 5.10: Illustration of a model for the test section of the line injection method.

shield under test is chosen as reference. The shield of the coax has a radius $r_{s}$, whereas wires 1 and 2 (injection wire and inner wire of the coax, respectively) have radii $r_{1}$ and $r_{2}$. Voltages in the two wires are defined with respect to the shield. In the exterior of the coax the current on conductor 1 has its return path via the outside of the shield. In the interior, conductor 2 has its return current on the inside of the shield. The MTL equations can be solved for currents and voltages once all properties of the transmission line have been defined.

Per-unit-length parameters In this case the inductance and capacitance matrices for the MTL system in Figure 5.10 are:

$$
\mathbf{L}=\left[\begin{array}{cc}
l_{11} & 0 \\
0 & l_{22}
\end{array}\right], \quad \mathbf{C}=\left[\begin{array}{cc}
\mu_{0} \varepsilon_{0} \varepsilon_{r e} & 0 \\
0 & \mu_{0} \varepsilon_{0} \varepsilon_{r i}
\end{array}\right] \mathbf{L}^{-1},
$$

in which:

$$
l_{11}=\frac{\mu_{0}}{2 \pi} \cosh ^{-1}\left(\frac{d^{2}-r_{s}^{2}-r_{1}^{2}}{2 r_{s} r_{1}}\right), \quad l_{22}=\frac{\mu_{0}}{2 \pi} \ln \left(\frac{r_{s}}{r_{2}}\right)
$$

For $l_{11}$ simplification to the natural logarithm is not applied since usually the injection wire is very close to the shield. The permittivity of dielectric material inside the coax is given by $\varepsilon_{r i}$. Similarly the exterior medium permittivity, if homogeneous, is represented by $\varepsilon_{r e}$. If the insulation of the injection wire is placed directly against the shield under test an effective permittivity can be estimated by $\varepsilon_{r e}$ of the insulation. 
The total p.u.I. impedance matrix of the MTL system carries both the ohmic losses in the wires, as well as the shield and transfer impedances and the inductance matrix defined above. It is given by the following expression:

$$
\hat{\mathbf{z}}=\left[\begin{array}{cc}
R_{c 1} & 0 \\
0 & R_{c 2}
\end{array}\right]+\left[\begin{array}{cc}
Z_{s h, e} & Z_{T} \\
Z_{T} & Z_{s h, i}
\end{array}\right]+j \omega \mathbf{L},
$$

in which $R_{c 1}$ and $R_{c 2}$ represent the losses in the two wires. These can be computed by equation (4.5) with $d_{g}$ and $\sigma_{g}$ changed to $2 r_{i}$ and the conductivity of copper, respectively. The transfer impedance is given by $Z_{T}$. Schelkunoff [62] gives a useful expression for the shield impedance of a solid shield, which is used here as estimation for impedances of interior and exterior shield impedance, respectively $Z_{s h, i}$ and $Z_{s h, e}$. For approximating the impedance of the braided shield by a solid copper shield an equivalent thickness is computed by assuming that the low-frequency value of the shield impedance is equal to the DC value of the transfer impedance. Finally, the conductance matrix $\mathbf{G}$ is assumed to be zero, yielding a p.u.l. admittance matrix equal to:

$$
\hat{\mathbf{Y}}=\mathrm{j} \omega \mathbf{C} \text {. }
$$

In practice, proximity effects will cause the current to be distributed in a non-uniform way throughout the cable shield. To minimize such effects in transfer impedance measurements sometimes multiple injection wires are used. The modelling approach described above neglects these proximity effects. It assumes that the current is distributed in a uniform way over the circumference of a shield.

Termination impedances The transmission lines are terminated as shown in Figure 5.10, with $R_{i}$ at both sides of the interior TL and $R_{e}$ in the exterior TL. A voltage source is included between the injection wire and the shield. This yields for the vectors containing voltage sources and the impedance matrices:

$$
\mathbf{V}_{S}=\left[\begin{array}{l}
1 \\
0
\end{array}\right], \quad \mathbf{V}_{L}=\left[\begin{array}{l}
0 \\
0
\end{array}\right], \quad \mathbf{Z}_{S}=\mathbf{z}_{L}=\mathbf{z}=\left[\begin{array}{cc}
R_{e} & 0 \\
0 & R_{i}
\end{array}\right]
$$

To avoid resonances in transfer impedance measurements, it is always attempted to have matching terminations. The interior and exterior transmission lines are designed to have a characteristic impedance equal to the measurement equipment. Therefore, we take the following terminations:

$$
R_{e}=\alpha \sqrt{l_{11} / c_{11}}, \quad R_{i}=\beta \sqrt{l_{22} / c_{22}},
$$

in which mismatches can be evoked by choosing $\alpha$ or $\beta$ unequal to one.

\section{Triaxial method}

The triaxial method for transfer impedance measurements, of which the test section is illustrated in Figure 5.11, is modelled in a similar way. The sample 


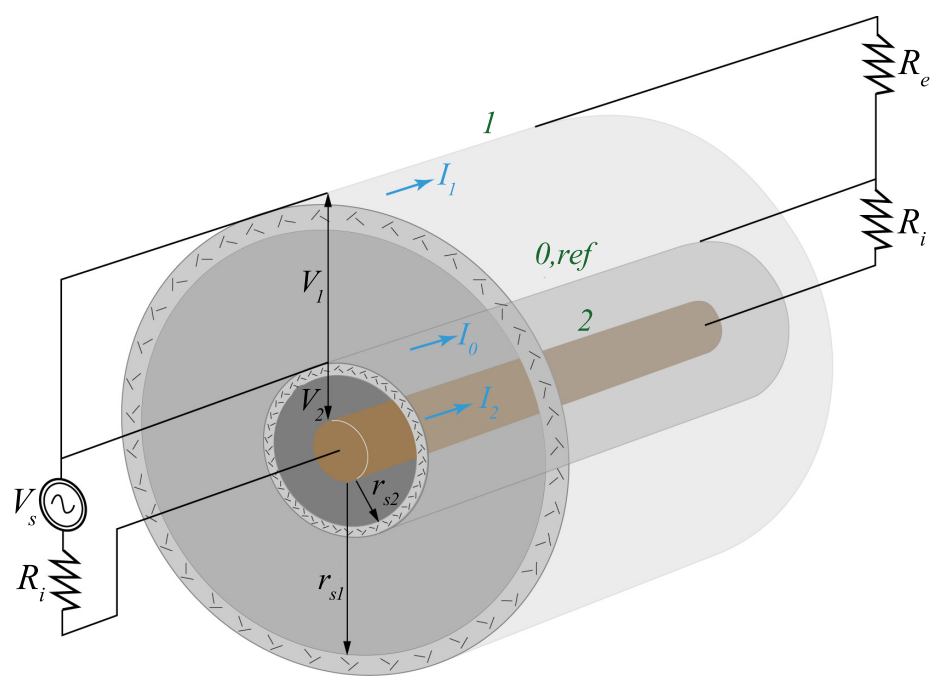

Figure 5.11: Illustration of a model for the test section of the triaxial method.

under test is enclosed by a copper tube, which introduces a difference in the inductance matrix:

$$
l_{11}=\frac{\mu_{0}}{2 \pi} \ln \left(\frac{r_{s 1}}{r_{s 2}}\right), \quad l_{22}=\frac{\mu_{0}}{2 \pi} \ln \left(\frac{r_{s 2}}{r}\right) .
$$

Moreover, the p.u.l. admittance matrix changes slightly. The resistance of conductor 1 in (5.12), $R_{c 1}$, should be replaced by the interior impedance of the solid copper tube enclosing the test sample in the triaxial setup. The computation of the capacitance matrix remains as given in (5.10).

Regarding the terminations of the MTL, as opposed to the line injection method, usually the wire inside the shield is excited and the induced voltage between the shield and copper tube is measured (see also Figure 5.11). Moreover, the near-end of the interior transmission line, the coax under test, is short-circuited in this measurement method. This results in:

$$
\mathbf{v}_{S}=\left[\begin{array}{l}
0 \\
1
\end{array}\right], \quad \mathbf{z}_{S}=\left[\begin{array}{cc}
0 & 0 \\
0 & R_{i}
\end{array}\right]
$$

The impedance matrix on the load side remains equal to (5.14).

\subsubsection{Transfer impedance computation}

After the transmission line has been characterised by the properties given in the previous section the MTL equations can be solved for voltages and currents in all conductors at any position. Since mismatches and propagation effects are included in the MTL model, extracting transfer impedance from 
the induced voltage at near or far-end leads to different results, as is the case in measurements (in the high-frequency region). In practice the induced voltage is usually measured at the far-end due to a larger frequency range of validity.

As before, $\mathbf{V}_{0}$ and $\mathbf{I}_{0}$ denote the vectors representing voltages and currents of all conductors at the near-end side. Similar quantities at the far-end side are given by $\mathbf{V}_{L}$ and $\mathbf{I}_{L}$. In this case the second entries of the voltage vectors, $V_{0,2}$ and $V_{L, 2}$, are the open source voltages induced at the near-end and the far-end of the coax, and $I_{0,1}$ and $I_{0,2}$ are the corresponding currents flowing at the source side of the two wires. For the line injection, $I_{0,1}$ is the induced source current, while for the triaxial method this is given by the current on the internal wire $I_{0,2}$ With these definitions the transfer impedance that would be measured in a line injection method can be computed in the following way:

$$
Z_{T, N}=2 V_{0,2} / l I_{0,1}, \quad Z_{T, F}=2 V_{L, 2} / \ell I_{0,1} .
$$

Here the subscripts relate to near-end or far-end. By a difference in terminations, for the triaxial method only the far-end voltage can be measured, yielding:

$$
Z_{T, F}=V_{L, 1} / \ell I_{0,2}
$$

For (5.18) and (5.19) currents and voltages at the ends of the MTL are required. These can be obtained by solving equations (2.6), (2.5) and [5]:

$$
\begin{aligned}
\mathbf{I}_{L} & =\boldsymbol{\Phi}_{21} \mathbf{V}_{S}+\left[\boldsymbol{\Phi}_{22}-\boldsymbol{\Phi}_{21} \mathbf{Z}_{S}\right] \mathbf{I}_{0} \\
\mathbf{V}_{L} & =\mathbf{Z}_{L} \mathbf{I}_{L}
\end{aligned}
$$

These voltages and currents can be solved explicitly by using low-frequency approximations for the chain parameter matrices $\boldsymbol{\Phi}$ as given by equation (2.10). The resulting closed-form expressions are only valid for electrically short wires, but transition frequencies roughly similar to the standards $[66,69]$ can be derived. Moreover, they lead to a first intuition on the cause of resonances in transfer impedance measurements. In the following these expressions are derived for the line injection method. Similar expressions can be derived for the triaxial method.

\section{Low-frequency closed-form expressions}

When substituting the low-frequency approximations of (2.10) into (5.20) the voltage induced in the coax under test can be approximated by:

$$
\begin{aligned}
& V_{0,2}=D^{-1} \ell Z_{T} R_{i}\left(1+\mathrm{j} \omega \ell R_{e} c_{11}\right) \\
& V_{L, 2}=-D^{-1} \ell Z_{T} R_{i}\left(1+\mathrm{j} \omega \ell R_{i} c_{22}\right)\left(1+\mathrm{j} \omega \ell R_{e} c_{11}\right),
\end{aligned}
$$

in which:

$$
\begin{aligned}
D= & \left(2 R_{e}+\ell Z_{s h, e}+\mathrm{j} \omega \ell\left(l_{11}+R_{e}^{2} c_{11}\right)\right)\left(2 R_{i}+\ell Z_{s h, i}+\mathrm{j} \omega \ell\left(l_{22}+R_{i}^{2} c_{22}\right)\right) \\
& -\ell^{2} Z_{T}^{2} .
\end{aligned}
$$


By manipulating (5.21) and recognising that in this case the shield and transfer impedance are much smaller than the termination impedances, it is possible to derive an approximation for the frequency at which the low-frequency expressions do not hold anymore: a transition frequency.

\section{Transition frequencies}

For transfer impedance measurements the validity range for the frequency is important. In the standards expressions are derived for maximum frequencies up to which the different methods can be used. Here, in a different way, similar expressions are derived that give insight in the behaviour of the measurement setups.

The transition frequency, at which higher order terms start to interact, is approximated for the near-end and far-end expressions in (5.21) by:

$$
\begin{aligned}
f_{N E} & \approx \frac{1}{\pi \ell} \frac{R_{e} R_{i}}{R_{e}\left(l_{22}+R_{i}^{2} c_{22}\right)+R_{i}\left(l_{11}+R_{e}^{2} c_{11}\right)} \\
f_{F E} & \approx \frac{1}{\pi l} \frac{R_{e} R_{i}}{R_{e}\left(l_{22}-R_{i}^{2} c_{22}\right)+R_{i}\left(l_{11}-R_{e}^{2} c_{11}\right)} .
\end{aligned}
$$

The fact that higher order frequency terms start to interact implies that neglecting propagation effects is not valid anymore, which is the criterion for the bounds on usability of the presented measurement methods in [66]. By using the termination impedances in (5.15) these expressions simplify to:

$$
\begin{aligned}
f_{N E} & \approx \frac{\alpha \beta c}{\alpha\left(1+\beta^{2}\right) \sqrt{\varepsilon_{r i}}+\beta\left(1-\alpha^{2}\right) \sqrt{\varepsilon_{r e}}} \\
& \approx \frac{1}{2 \pi \ell} \frac{c}{\sqrt{\varepsilon_{r i}}}, \quad \text { if } \quad \alpha=\beta=1 \\
f_{F E} & \approx \frac{\alpha \beta c}{\alpha\left(1-\beta^{2}\right) \sqrt{\varepsilon_{r i}}+\beta\left(1-\alpha^{2}\right) \sqrt{\varepsilon_{r e}}} \\
& \rightarrow \infty \text { if } \alpha=\beta=1 .
\end{aligned}
$$

The final approximations yield the transition frequencies for perfect termination of the MTL. The near-end expression is similar to that in [66], though the permittivity of the exterior transmission line is not present here while it is in that standard. When permittivities of interior and exterior transmission lines are equal, the near-end expression in (5.24) is equal to that in the standards [66]. The far-end transition frequency approaches infinity with perfect terminations. For equal permittivities inside and outside the coax this again coincides with [66]. Possible resonances caused by differences in propagation speeds can only be obtained from the complete MTL solution since these propagation effects are contained only in the higher order terms, which illustrates a limitation of the low-frequency analysis methods. 


\subsubsection{The effects of dielectrics and mismatches}

The MTL models for transfer impedance measurements described in Section 5.2.1 can be utilised to determine the sensitivity with the various model parameters. The focus in this section is to investigate the effects of dielectrics and mismatches to the measured transfer impedance. Firstly, the following cases are analysed:

- Comparison of measurement setups with fixed terminations and dielectric permittivity

- Fixed terminations with varying dielectric permittivity

- Varying terminations with fixed dielectric permittivity

In these cases the dielectric properties and terminations of inner and outer transmission lines are varied. All other parameters are fixed to specific values: shield radius $r_{s}=3 \mathrm{~mm}$, inner conductor radius $r_{2}=0.8 \mathrm{~mm}$, injection wire radius $r_{1}=0.51 \mathrm{~mm}$, separation distance $d=4.01 \mathrm{~mm}$ and length of the transmission line equal to $1 \mathrm{~m}$. For comparison also two triaxial results are given.

After the analysis of these parameter variations, the procedure introduced at the start of this chapter is followed to determine the actual sensitivity with respect to dielectric and mismatch parameters. The output for which sensitivities are computed is the far-end measured transfer impedance, since this method is more commonly used, for reasons given in the sections below. In all simulations, the input transfer impedance is generated by the BEtter Analysis of TRansfer Impedance of Cable Shields (BEATRICS) model [39], and is plotted in Figure 5.12.

\section{Comparison of measurement setups - fixed terminations and permittivi- ties}

Consider the situation in which both the internal and external circuit are terminated with perfectly matched impedances, e.g. $\alpha$ and $\beta$ in (5.15) are equal to one. Moreover, the interior and exterior permittivites are fixed to $\varepsilon_{r i}=2.5$ and $\varepsilon_{r e}=1$. Simulation results for the closed-form transfer impedance, as well as the transfer impedance obtained in the line injection and triaxial model are given in Figure 5.13. All results are given for both near-end and far-end, except for the triaxial case, in which only far-end evaluation is possible due to the short circuit at near-end. The blue line represents the input transfer impedance used in (5.12), which is computed by the BEATRICS model. This line is covered by the closed-form solution for far-end transfer impedance, given in green. This result is obtained by the ratio of far-end voltage in (5.21) and a similar expression for the injection current. These closed-form expressions are only valid for electrically short cables. Nevertheless, the near-end expression does show the trend caused by the long line effects, causing a maximum in measured transfer impedance. 


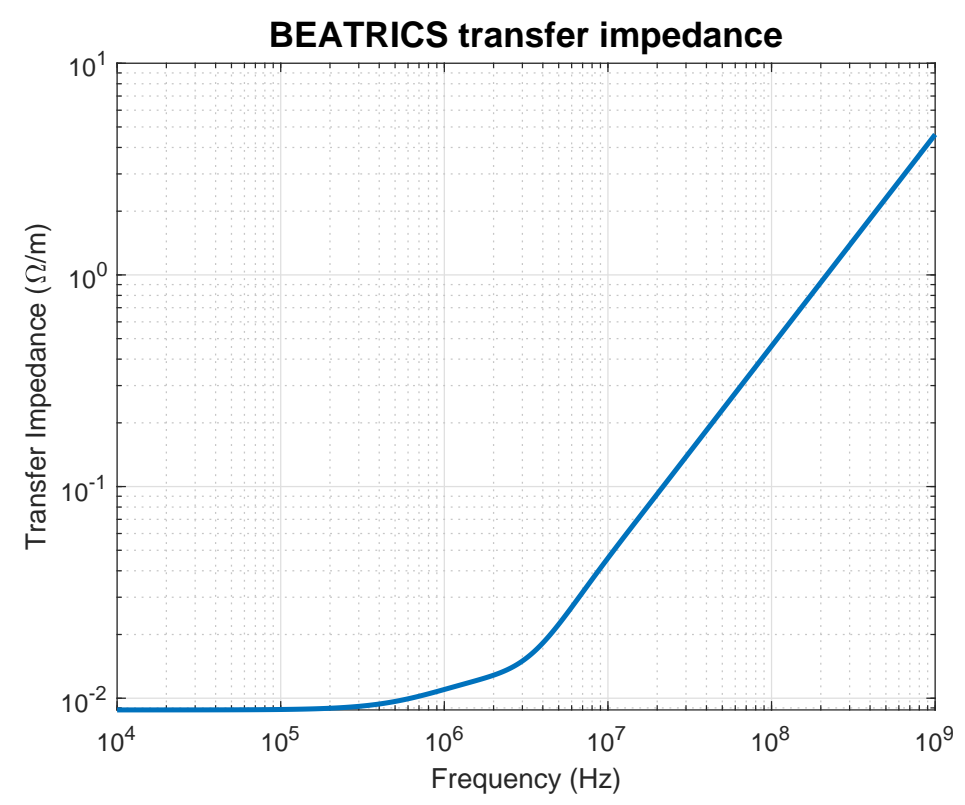

Figure 5.12: Input transfer impedance for MTL sensitivity analysis.

Comparing the MTL simulations for the near and far-end line injection measurements, the results clearly illustrate the fact that far-end measurements are valid up to higher frequencies, since the resonances caused by differences in propagation speeds in the interior and exterior transmission line occur at a later instance. For the far-end setup, the triaxial result is also shown in Figure 5.13. Apart from the clear resonance caused by different propagation speeds this result shows extra fluctuations, which are a consequence of the inherent mismatched terminations, since the near-end of the exterior circuit is shortcircuited. This causes the presented triaxial setup to be applicable up to lower frequencies than line injection, which is consistent with standards and literature $[66,72]$.

Since in practice far-end setups are more frequently used by their higher frequency range of applicability, the following sections will show only far-end results.

\section{Characteristic terminations - Varying dielectric permittivities}

Figure 5.14 shows simulated far-end transfer impedance results when the permittivity inside the shield under test is changed to $\varepsilon_{r i}=2.5$ and $\varepsilon_{r i}=4$, while $\varepsilon_{r e}$ is kept fixed to 1. The terminations are kept perfectly matched. For $\varepsilon_{r i}=2.5$ this coincides with the $50 \Omega$ often used in practice.

The change in interior relative permittivity from 2.5 to 4 yields higher differences in the propagation speeds of the interior and exterior transmission 


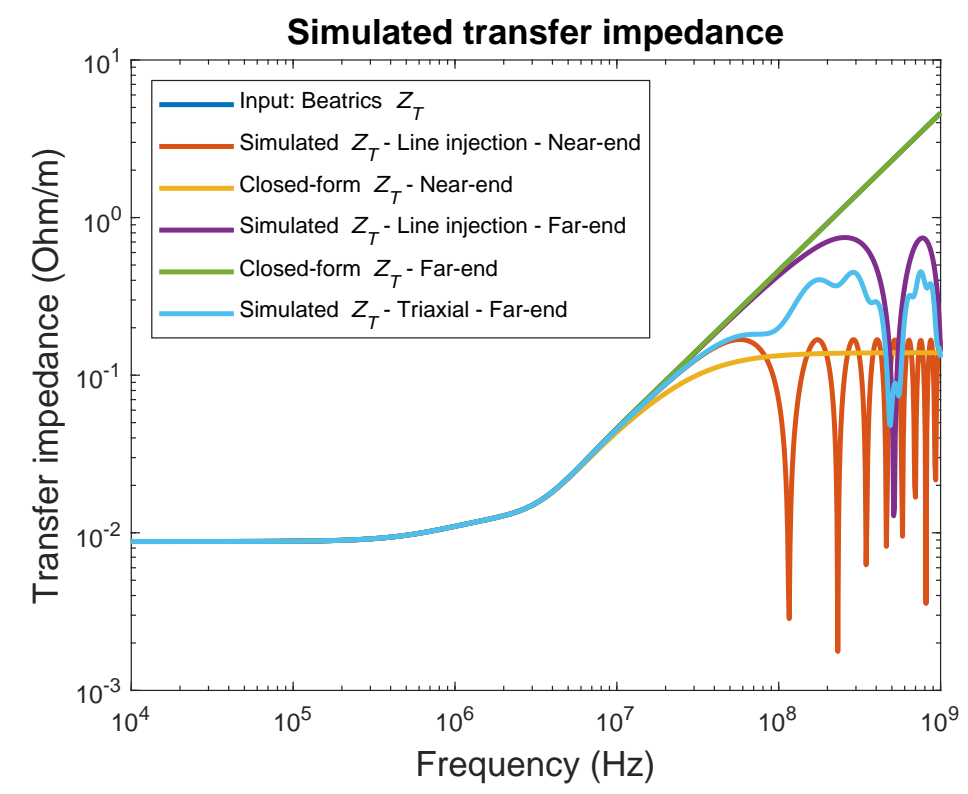

Figure 5.13: Simulation results for the line injection and triaxial method with characteristic terminations and $\varepsilon_{r i}=2.5$ and $\varepsilon_{r e}=1$. Closed-form solutions are also shown, of which the far-end result is exactly equal to the BEATRICS input.

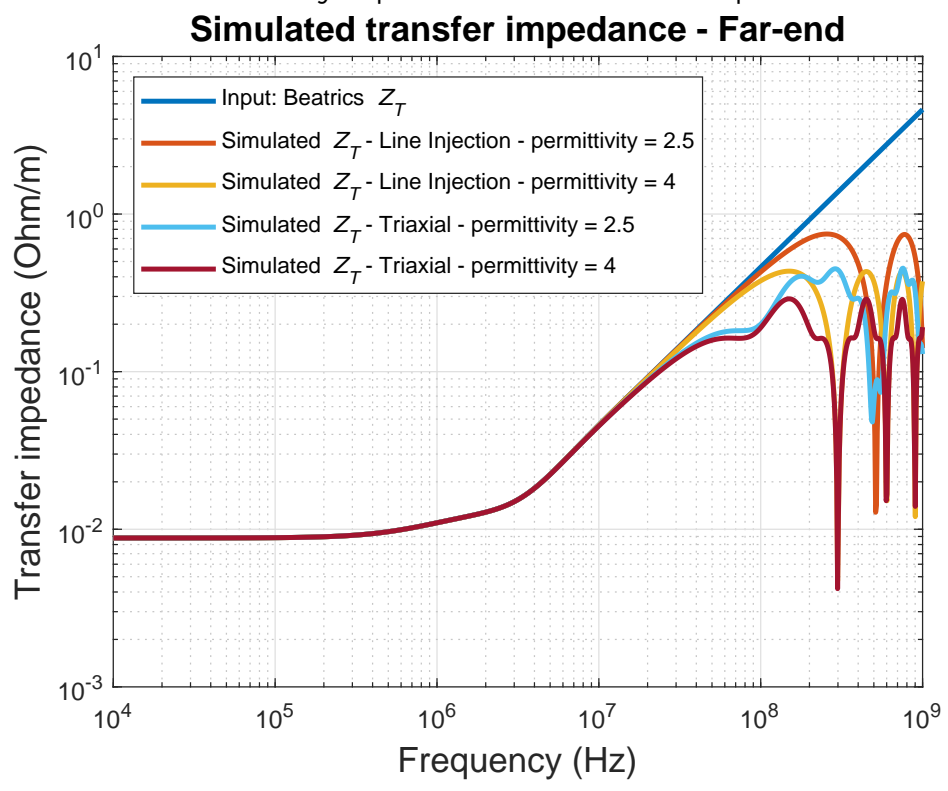

Figure 5.14: Simulation results for the line injection and triaxial method with characteristic terminations, $\varepsilon_{r e}=1$ and varying interior permittivity. Only far-end results are shown. 
lines. The results in Figure 5.14 show that this increase leads to an earlier resonance in the measured transfer impedance, making the setup usable up to lower frequencies. Again, results for both the line injection and triaxial methods are shown, and a similar conclusion on permittivity dependency holds for both methods. This is confirmed by the analytical expressions for null frequencies $[66,72]$. These show that indeed for far-end measurements the applicability of the measurement setups depend on the difference between $\varepsilon_{r i}$ and $\varepsilon_{r e}$, which increases if the permittivity is changed from 2.5 to 4 .

\section{Fixed dielectric permittivities - Varying mismatched terminations}

The effect of mismatches on the measurements of transfer impedance can be simulated by changing the values of $\alpha$ and $\beta$ in (5.15). Since matching of the coax is usually easier than matching of the injection wire in case if line injection, here mismatches in the exterior domain are investigated. In Figure 5.15 results are presented when the value for $\alpha$ is varied from 1 (matched case) to 0.75 and 0.5 (mismatched cases). The exterior termination is matched $(\beta=1)$ and $\varepsilon_{r i}=2.5$ and $\varepsilon_{r e}=1$. Clearly, when introducing an increasing mismatch (decreasing $\alpha$ ) fluctuations observed on top of the resonance effects caused by the differences in interior and exterior propagation speeds, increase. However, the trends of measured transfer impedances are not changed much by the increasing mismatches. Therefore, ensuring equal propagations speeds in and outside of the coax seems to be more critical to achieve a good estimation of inductance values for transfer impedance than matching the exterior transmission line.

\section{Sensitivity analysis}

For the case with mismatched terminations and dielectrics, sensitivity with respect to the termination impedances and dielectric constants is analysed. Firstly, the material properties for the inner and outer transmission lines are fixed to $\varepsilon_{r i}=2.5$ and $\varepsilon_{r e}=4$, while the terminations of the internal and external circuits are both $50 \Omega$, which coincides with $\alpha=1.16$ and $\beta=1$. These two parameters are separately varied with those mean values and a standard deviation of $0.1 \mu$, for which the results are shown in Figure 5.16 and Figure 5.17.

Figure 5.16 shows both sensitivity and transfer impedance statistics for variations in $\beta$ (and $\alpha$ fixed to 1.16), which governs impedance mismatches in the internal transmission line. As before, the horizontal and dashed lines in these sensitivity plots indicate the different regions explained in Figure 5.2. As expected, for low frequencies there is no influence of mismatches to measured transfer impedance. Only for higher frequencies, where the resonances start to occur, there is an effect of impedance mismatch. However, for mismatches with standard deviation of $10 \%$ of the characteristic impedance of the interior line, the influence is still small, as is observed from Figure 5.16b. For $\beta>1$ the 


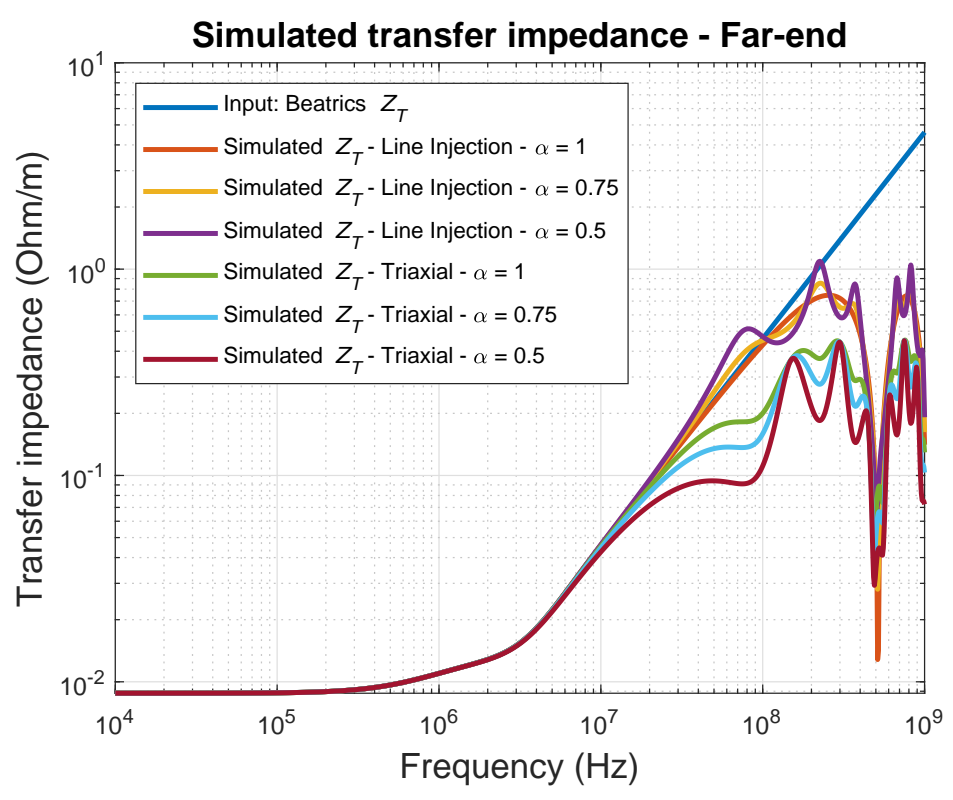

Figure 5.15: Simulation results for the line injection and triaxial method with $\varepsilon_{r i}=2.5$ and $\varepsilon_{r e}=1$, while the terminations of the interior transmission line are matched and the exterior terminations are varied. Only far-end results are shown.

measured transfer impedance will be slightly larger than the analytical value, and for $\beta<1$ results will be smaller.

Figure 5.17b shows the sensitivity with respect to the exterior line terminations. That is, the parameter $\alpha$ has been varied and $\beta$ is fixed to one to obtain these results. Again, for low frequencies the sensitivity is negligible, but for high frequencies these termination impedances do have influence to the measured transfer impedance. As opposed to the interior terminations, a deviation from the exterior characteristic impedance causes fluctuations of the measured result around the analytical transfer impedance, while the slope of the trend with respect to frequency is not affected.

For the sensitivity with respect to material properties, the termination impedances are now fixed to (5.15) with $\alpha=1.16$ and $\beta=1$. Figure 5.18 gives sensitivity and statistic results for the case in which the interior material is changed and $\varepsilon_{r e}=4$. The sensitivity with respect to the exterior permittivity is similar and not shown here. At the frequency where long line effects start to play a role, the sensitivity is comparable to that in Figure 5.17b. However, towards the first resonance the sensitivity with respect to the permittivity increases rapidly. This is caused by the fact that by a change in interior permittivity, the difference in propagation speeds of interior and exterior transmission lines is changes. At its turn, this shifts the resonance yielding high sensitivities for the transfer impedance at those frequencies. Figure 5.19 shows multi- 
ple SROM simulations that were input to the computation of sensitivity, and these results confirm that shift in resonance frequency. Indeed, from theory it is known that the first resonance in measured transfer impedance occurs at [61]:

$$
f_{r}=\frac{1}{\left\|v_{1}^{-1}-v_{2}^{-1}\right\| \ell} .
$$

Here $v_{1}$ and $v_{2}$ are the propagation speeds outside and inside the coaxial cable, respectively. Clearly, when these are equal, there will be no resonance. The greater the difference in permittivities, the earlier a resonance will occur and this will also cause the sensitivity to increase, for the case shown in Figure 5.19 to nearly $20 \mathrm{~dB}$ at $1 \mathrm{GHz}$.

Summarizing, Table 5.1 gives the sensitivity in MTL simulations of measured transfer impedance with respect to the four parameters that were investigated. These values are all for a frequency of $1 \mathrm{CHz}$. Clearly, the measurement results are more sensitive to a deviation in permittivity of material than they are for a change in termination impedance relative to characteristic impedance.

Table 5.1: Sensitivities of transfer impedance at a frequency of $1 \mathrm{GHz}$.

\begin{tabular}{lll}
\hline \hline Parameter & Transmission Line & Sensitivity \\
\hline Termination impedance & Interior & $-5.7 \mathrm{~dB}$ \\
& Exterior & $-5.8 \mathrm{~dB}$ \\
Permittivity & Interior & $11.5 \mathrm{~dB}$ \\
& Exterior & $14.7 \mathrm{~dB}$ \\
\hline
\end{tabular}

\subsection{Summary}

In this chapter MTL models were applied to perform sensitivity analysis in two cases:

- Crosstalk between twisted pairs in an overshield

- Transfer impedance measurement methods

Based on theory used throughout this thesis, MTL models are created for these cases. The Stochastic Reduced Order Model is then applied to perform sensitivity analysis. This SROM method can reduce computation times by a factor in the order of a thousand when compared to the Monte Carlo method.

The first case considered comprises to two cable bundles with either two or seven twisted wire pairs. The sensitivity results have been compared to that of the Monte Carlo method. For cross-sectional parameters the result is very accurate in the entire frequency range. For longitudinal parameters like the 


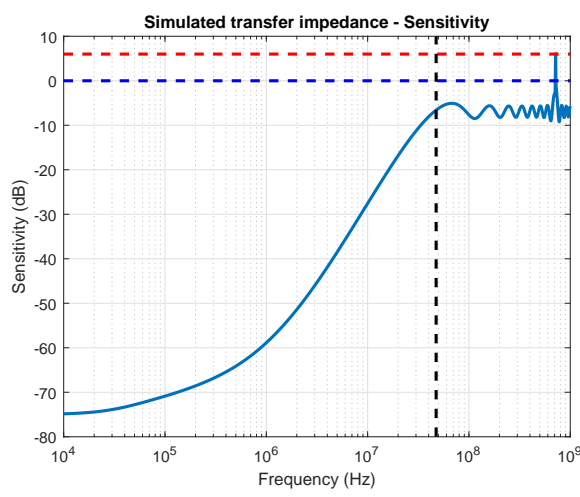

(a) Sensitivity.

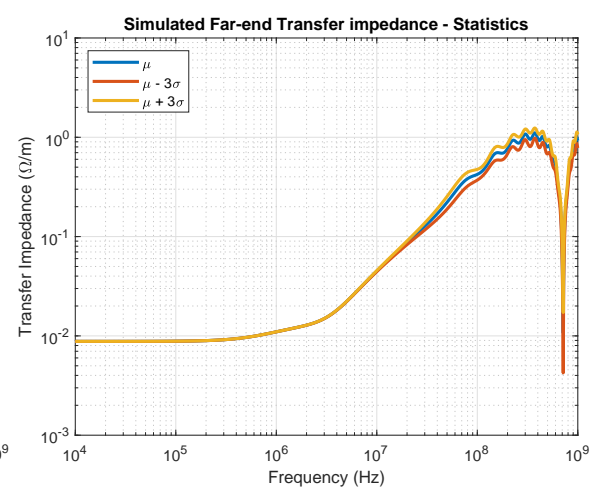

(b) Transfer impedance statistics.

Figure 5.16: Simulated sensitivity results with respect to $\beta$.

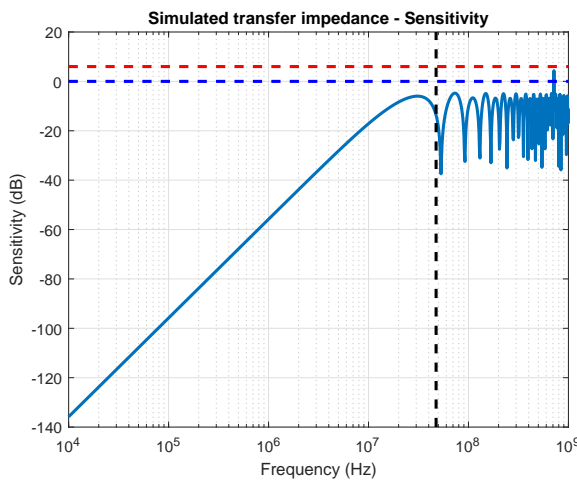

(a) Sensitivity.

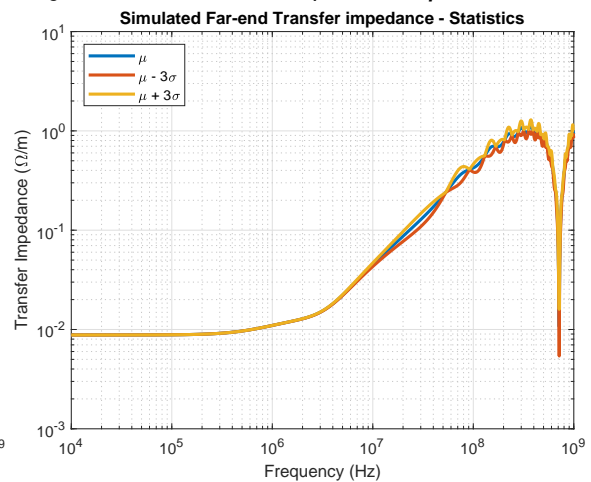

(b) Transfer impedance statistics.

Figure 5.17: Simulated sensitivity results with respect to $\alpha$.

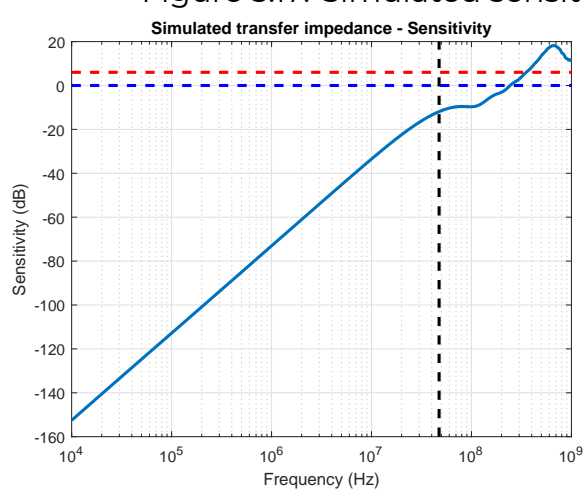

(a) Sensitivity.

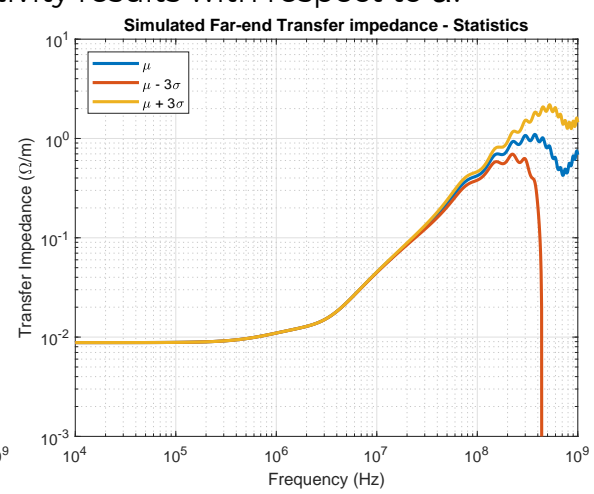

(b) Transfer impedance statistics.

Figure 5.18: Simulated sensitivity results with respect to $\varepsilon_{r i}$. 


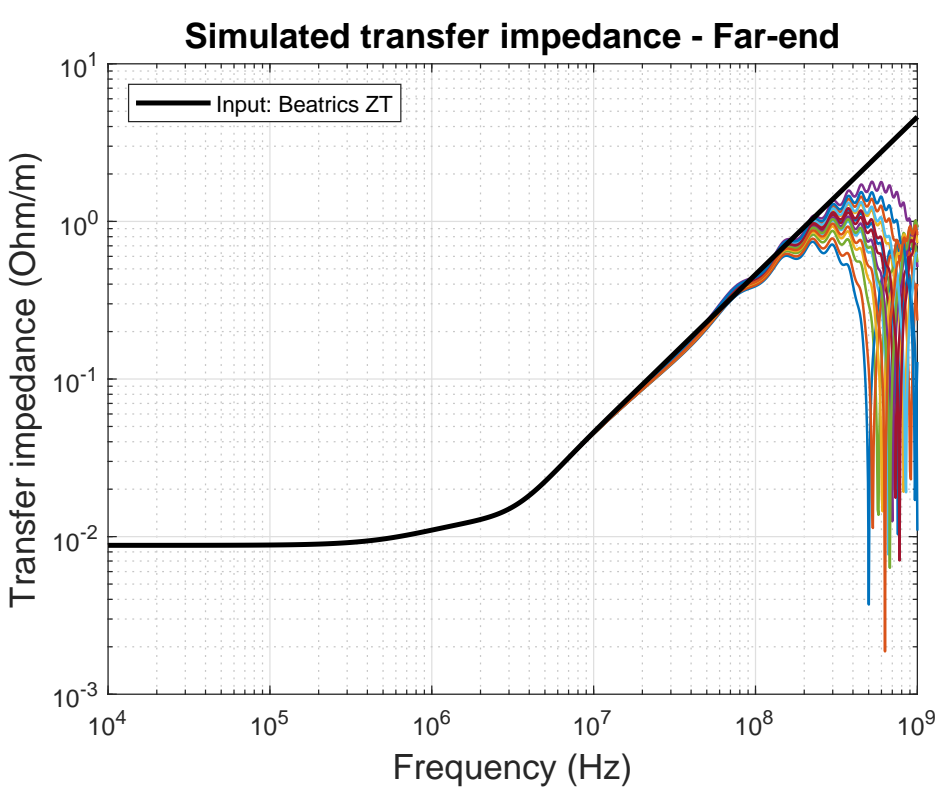

Figure 5.19: SROM simulations and transfer impedance input (black) for variation in $\varepsilon_{r i}$ around 2.5 , and $\varepsilon_{r e}=4$

twist rate, results of the SROM method appear to be slightly unstable with variations of a few dB. However, average crosstalk is computed accurately and equal to that of $\mathrm{MC}$ simulations. Estimation of the standard deviation appears to be slightly less accurate for such cases.

Sensitivities with respect to the twist rate of twisted wire pairs can be very high. Both these sensitivities and the average near-end crosstalk can be greatly reduced by using different twist rates for the culprit and victim transmission lines. Therefore, creating cable bundles with many different types of twisted pairs would be good from a crosstalk perspective.

The influence of bundle twisting and the meandering of individual cables along the length of the bundle were discussed. For the case of twisted pairs with equal twist rates the bundle twist and cable meandering have no influence on the sensitivities with respect to inter-pair and intra-pair separation distance. Only in the high-frequency area minor changes in the intra-pair separation sensitivity are observed when cable meandering is included. For the estimation of average crosstalk in case of twist rate uncertainty, the bundle twist and cable meandering do not affect the results when twist rates are equal. If victim and culprit TWP have unequal twist rate, bundle twist does not change average crosstalk, but cable meandering should be included in the model, since it increases the estimated average near-end crosstalk.

The second case considered deals with MTL simulation of two different transfer impedance measurement methods: the line injection method and 


\subsection{SUMMARY}

the triaxial method. By definition, transfer impedance is a quantity defined over an infinitesimal piece of the shield, for which various analytical models exist. However, when transfer impedance is measured this will always be done on a sample with a finite length, which will introduce transmission line effects. The MTL simulations are able to predict such behaviour in these measurements. Moreover, closed-form expressions are extracted that lead to some transition frequencies similar to the standards, which are useful to investigate the origin of resonances in transfer impedance measurements.

Sensitivity analysis with respect to the terminations of the transmission lines and dielectric permittivities are performed. These show that the most sensitive parameter is the dielectric permittivity. A larger difference in relative permittivities of the interior and exterior transmission line will enforce larger deviations in propagation speeds of the two lines, causing resonances to occur earlier in the frequency domain. A mismatch in terminations only causes small fluctuations on top of the previously mentioned behaviour; the trend with frequency remains similar. Therefore, ensuring that the permittivities of the interior and exterior transmission lines are closer in value will extend the range of applicability of the measurement setup and ease the estimation of inductive properties of transfer impedance. 
CHAPTER 5. APPLICATIONS OF CROSSTALK MODELS 


\section{6 | Conclusions and future research}

Sustainable aviation is inescapable, and consequently so is the electrification of an increasing amount of on-board systems, for which connections have to be provided as part of the EWIS. The quest for weight optimisation of EWIS requires crosstalk analysis in many design stages and iterations. This thesis has provided alternatives to time-consuming full-wave solutions or full-detail MTL equations. The focus has been on the development of methods that yield crosstalk predictions and are useful for incorporation in optimisation procedures and decision-making processes for early risk assessments. Specifically, one chapter was dedicated to each of the four goals set out in Section 1.2. Their conclusions are given in this chapter.

\section{Chapter 2}

The second chapter of this thesis has introduced low-frequency methods to derive closed-form expressions for crosstalk in MTLs, which was identified as the first goal in Section 1.2. Application of this method has resulted in expressions for crosstalk between wire pairs close to a perfectly conducting ground plane or without nearby ground plane. The closed-form expressions clearly relate crosstalk levels to all designable parameters and show in one instant to which one crosstalk is most sensitive. For the cable configurations that were analysed, separation distance is the most relevant parameter, especially when a ground plane is included. In that case the change of crosstalk can be up to 24 $\mathrm{dB} / \mathrm{dec}$ de for a change in separation. The derived crosstalk expressions were also converted into a clear overview of all parameter dependencies, which can serve as design rule in the design processes of EWIS. Finally, another application of the low-frequency approximations was found in the speed-up of crosstalk computations for non-uniform cables. By applying the low-frequency alternatives to uniform cascaded sections, speed-ups in the order of a factor 20 in computation times can be achieved.

Testing the given expressions for their applicability as actual practical EWIS design rules is considered future research. To that extent there is a need to fur- 
ther test the validity boundaries for more complex cases. It has already been shown that the addition of extra nearby cables has little effect on the parameter dependencies, but there are more complications imaginable, such as violation of the geometrical assumptions used in Chapter 2 or addition of bundle shields that will serve as reference instead of the nearby ground plane.

\section{Chapter 3}

This chapter has introduced generic predictions of crosstalk for cables that involve shielding, which is used in great quantities in aircraft wiring for protection against crosstalk and external interferences. Low-frequency methods introduced in Chapter 2 again yielded closed-form expressions for crosstalk between for instance an unshielded wire and a double shielded wire. Results show clear distinctions in frequency behaviour caused by the shields. Further simplification of the expressions has provided generic predictions that include crosstalk levels and transition frequencies for each frequency region, yielding the results for the second goal indicated in Section 1.2. Readily available parameter dependencies for these expressions show which phenomenon of the shield is responsible for which effect in crosstalk behaviour.

Applicability of the obtained generic crosstalk predictions can be found in understanding the use of cable shields, as well as optimisation of EWIS weight versus desired protection against interference by shielding. Tuning shields such that suppression of crosstalk is achieved in specific frequency regions can be based on generic predictions. Further maturation towards such optimisations is considered future work. This also includes generic predictions of other cable configurations, involving for instance shielded wire pairs. In principle the coupling mechanism between such cables is still common-mode and thus predictions can be based on the ones available for coaxes. However, specifically the presence of imbalances in terminations and geometry of shielded wire pairs and the corresponding effects on crosstalk behaviour should be part of future research.

\section{Chapter 4}

The third goal of this thesis involves crosstalk analysis in the vicinity of CFRP ground planes. To enable the analysis of crosstalk configurations in all modern and future aircraft, two novel methods have been introduced in Chapter 4 that are based on the MTL models, but allow the incorporation of ground planes with finite conductivity and thickness, such as CFRP fuselage panels. The first method, MTL-DG, utilises a discretisation of the ground plane in a finite array of cylindrical wires. The lossy properties can be incorporated by analytical expressions for the losses in cylindrical conductors. The second method, MTL-GI, incorporates a ground impedance matrix that has been derived from available 
expressions for stratified earth. Both methods have been applied to the case of crosstalk between wire pairs that are close to a CFRP ground plane. Moreover, the MTL-DG method has also been applied to wire pairs between two CFRP ground planes. In all cases the presented models have been validated against full-wave MoM simulations and measurements, yielding an excellent match in crosstalk results. However, for the developed MTL models, computation times on a simple laptop can be from 900 up to 70,000 times faster when compared to Method of Moment simulations on a 10-core Linux server.

Measurements and simulations have both confirmed the observation that replacement of aluminium ground planes by composite alternatives introduces an interesting switch in crosstalk behaviour in the frequency domain. Both finite thickness and conductivity of the ground plane are important parameters in this change of behaviour. The transition appears around the frequency for which the skin depth of the material is in the same order as the thickness of the ground plane. Below this frequency, EM fields penetrate uniformly through the CFRP ground plane, return currents distribute broadly under the wires, and crosstalk levels are similar to that between wire pairs without nearby ground plane. Above this frequency, the fields are unable to penetrate the CFRP further than one skin depth. Therefore, return currents concentrate more at the surface and below the current-driving wire and consequently crosstalk levels are equal to those for aluminium ground planes.

Further research should include investigations of the possibility to adapt the MTL-GI method to cases with cabling between two composite ground planes. It will include the use of for instance a Laplace solver to estimate p.u.l. parameters, as well as adaptation of the ground impedance matrix. Moreover, it is recommended to research the limitations of the given MTL models for lossy ground planes, especially when in case of wires between two ground planes these are placed very close to each other. This results in computationally tedious and challenging tasks for full-wave solvers. Moreover, there is a huge challenge when comparing simulation results for such cases with measurements, since placing the ground planes so close to each other yields very low crosstalk levels that might well be below the dynamic range of the measurement setup. However, validation is required, since the application of analytic wide-separation formulas for inductance and capacitance is prone to yield errors in crosstalk predictions when the separation of wires and ground conductors become smaller than 2 or 3 wire radii. Further research should indicate if, and if so at which point, numerical approximation of the p.u.l. becomes inevitable.

\section{Chapter 5}

The final chapter of this thesis focused on the fourth goal set out in Section 1.2 and has applied MTL models developed in earlier chapters to perform sensitivity analyses. Two cases were considered, being crosstalk between twisted 
wire pairs in shielded cable bundle and the simulation of transfer impedance measurement methods. In both cases SROMs have been applied to obtain the mean and standard deviation of an output parameter for a certain change in input parameters. This statistical method is much more efficient than for instance the well-known Monte Carlo method, in the sense that it uses only a small but smartly chosen subset of the roughly 10 to 100 thousand samples required for Monte Carlo simulations.

Sensitivity analyses have first been performed for crosstalk between twisted pairs in a shielded cable bundle. Sensitivity of several parameters has been investigated, among which intra-pair and inter-pair separation distances and twist rates. It appeared that the SROM method yields results that match very well with MC simulations. Only for the case in which different twist rates were applied to the twisted pairs some variability in the results was observed for the sensitivity with respect twist rate. For this case, average crosstalk predictions are equal for the SROM and MC method, however there is some instability in the estimation of standard deviations.

Even though for aviation industry the application of twisted pairs with equal twist rate is very realistic, the sensitivity analyses in Chapter 5 has revealed that it would be good practice to use a variation of twist rates, since this reduces average crosstalk as well as sensitivity with respect to twist rates. In general, the twist rate is a parameter yielding very high sensitivity. Finally, the effects of bundle twist and cable meandering to the sensitivity results have been investigated. Only in case of the estimation of average crosstalk between twisted pairs with unequal twist rates cable meandering has some impact. For all other cases, bundle twist and cable meandering has negligible effect on parameter sensitivities.

In the second case of sensitivity analysis, the MTL models of shielded cables introduced in Chapter 3 have been applied to investigate effects of changes in the setups for line injection and triaxial transfer impedance measurement methods. The analysis showed that mismatches in termination impedances and a difference in interior and exterior material permittivity have effects to transfer impedance measurement that can be distinguished. Differences in relative permittivity of the interior and exterior transmission line of the measurement setup cause differences in propagation speeds of those lines. A larger difference in propagation speeds will ensure an earlier first resonance in the measured transfer impedance. Therefore, in the high frequency area the measured transfer impedance is highly sensitive to a change in relative permittivity. Termination mismatches will result in smaller fluctuations around the general trend of transfer impedance, making the measured transfer impedance less sensitive to the corresponding parameters.

Further research could include the investigation of the effects that an error in transfer impedance measurement would have on crosstalk predictions. Moreover, sensitivity analysis can be applied in broader cases that are realistic in aircraft wiring systems, with the aim to further validate newly developed design rules for EWIS in future aircraft. 


\section{7 | Acknowledgements}

After graduating from Applied Mathematics at the University of Twente I was on the edge of a difficult choice between a PhD position or walking away from University. I chose to explore the unknown and consider myself grateful for finding a great new challenge at the Royal Netherlands Aerospace Centre. This proved to be the best of both worlds, positioned in between universities and industry. I made this choice knowing that I had never shut the other door indefinitely. Indeed, my work at NLR seemed to pave another way towards a PhD, but it wasn't without the help of others. I would like to take this opportunity to thank those.

First of all, I would like to thank my promotor Frank. We know each other through the EMC Europe conferences and in Wroclaw (2016) I asked you whether I could turn my work at NLR into a PhD under your supervision. Your answer was plain and simple: yes you can! Five years later and here we are! Thanks for giving me that opportunity and providing me with everything I needed. You've challenged me in many ways, whether it be motivating my novelty, or even outside my PhD by evoking projects regarding electric flight between UT and NLR. Luckily we are not done cooperating yet!

Another important factor in the start of my PhD has to be Harmen Schippers. Harm, you've been a great mentor in my years at NLR. I remember that you took around two hours in my job interview to tell me about your work at $N L R$, in the most enthusiastic way imaginable. You've managed to challenge me from day one, both in our work as well as during our running-breaks! Not only did I learn from your mathematical experience, but also from your ways of tackling complicated research problems and from the way you use your huge network to your advantage. You've guided my way into the conference world and introduced me to many people from your network. And on top of that, you planted the seed for this promotion. But most important of all, we had fun! There was no doubt on having good food and some beers when we were travelling. And whenever there was this Friday-afternoon "I need weekend" feeling: "then you should do something fun! Oh, and turn on some music"! Thanks Harm.

I would also like to thank all other colleagues from my team at NLR for fruitful discussions and of course their contribution to the measurements in this thesis. One more colleague in particular that I would like to thank is Jaco 
Verpoorte. You had the challenge of educating me, mathematician, from the electrical engineering side of things. When I came in my knowledge didn't extend much further than "impedance, isn't that like complex resistance?!" By now, luckily I past that station by a couple of miles. I've always enjoyed the plenty sparring sessions we've been having, and I never not came to you whenever I felt my questions could potentially be stupid. Thanks for that, as well as for all the non-work talks on photography! Finally, from NLR I have to make mention to my managers Michel Keuning, who was the first to support my ambition for a PhD by making it happen internally, and Erik Wegkamp to enable me for the final mile.

Dr. Niek! At the University of Twente you felt like a brother in "crime". We first met in Dresden, 2015, and we immediately made sure Harm's wife could be proud for not letting down the "young guys" by closing down the pubs with us in the early hours. The year after, in Wroclaw (where I won that best paper award, or wait...), more and more conference participants knew what the place to be was for networking (especially Dwi). The fun proceeded, in Angers, Amsterdam, Singapore, Barcelona, Prague, Budapest... and you trusted me travelling around with two of your friends who turned out to be more than nice dudes! Moreover, you were never short of listening ears and ideas when it came to discussions on the actual content. I'm happy to know that we have much more joint work and of course dissemination activities to go!

The University of Twente felt like a tight group, even for someone who was there on irregular basis. Laughter could always be found in the coffee corner, for which I can thank any member of the group. Robert in particular, thanks for always being in for beers anywhere in the world, and thanks for never not confusing me with other (post-)PhD candidates. Your advice during the writing of my thesis was much appreciated. Lilian, I'm sure many said it before, but you most certainly are the mother of our group. Without you many of us would have run away, but more importantly, things would not have been as 'gezellig' as they are! I may not have asked as much as others from you, but whenever I needed something, I just had to ask. Thanks for the good coffee talks and the always cheerful 'heeeeeeey' when I sat on our couch during coffee. Also thanks for always passing on hotel info after booking, so that I could book exactly that same one.

Friends are one of the most important things in life - for distraction, for celebrating the good things in life, and for sharing frustrations. Rob and Michiel, you've always shown so much interest in and enthusiasm about my work. I'm never short of listening ears when explaining the complicated stuff in laymen's terms to you, thanks for that. One of the biggest challenges after University was me moving to a new environment, Zwolle, where I knew very few people. Luckily, I never lost touch with University friends like Emma en Ellen, but also the "Eighth Heaven" who still served me food in my first working months and with whom I still very much enjoy our boardgame weekends. Music has been a huge part of my life as well, and still being able to write, as well as loose some energy and frustration in practice together with long time buddy Pieter feels 
good. Moreover, Rens has been a huge part of the earlier days in band life, and I thank you a lot for assisting me with the looks of this thesis. Finally, in Zwolle, I really want to thank Yannick, for becoming such a good friend and helping Zwolle become my new home town. Besides that, futsal at Exstudiantes, as well as the many friends I shared music with, gave me energy to go on.

The final period of my PhD has been enriched by another fantastic human being. Robin, thanks for never complaining when I wanted to work in our free time and for putting up with my periods of stress. Thanks for always being proud and letting me be entirely myself. Thanks for being you - happy, and quite often crazy. We've recently bought a house together, and I'm really looking forward to the upcoming years!

Last but definitely not least, my ever loving and supporting parents. You have always supported me in all my actions, including this five-year trip. Moreover, you provided me with everything to get here; in terms of a worriless youth, education, love and so much more. Thanks for the endless chats and help with all my tough (and not so tough) decisions in life. You could not have done any better and I hope to be able to reproduce that achievement in the future. I'm 'onmeunig' grateful to have you. 
CHAPTER 7. ACKNOWLEDGEMENTS 


\section{8 | Biography}

Jesper Lansink Rotgerink was born in Hengelo, the Netherlands, in July 1989. He graduated from the Bachelor Applied Mathematics in 2010 and from the master Applied Mathematics in 2013, both at the University of Twente. His master's thesis was a contribution to research into Alzheimer's disease. An attempt was made to optimise a mathematical model of a neural network to fit measured data of braincells infected with Amyloid $\beta$, the enzyme believed to cause Alzheimer's disease.

Since graduation Jesper started working at the Royal Netherlands Aerospace Centre (NLR). Being part of the Electromagnetic Technology

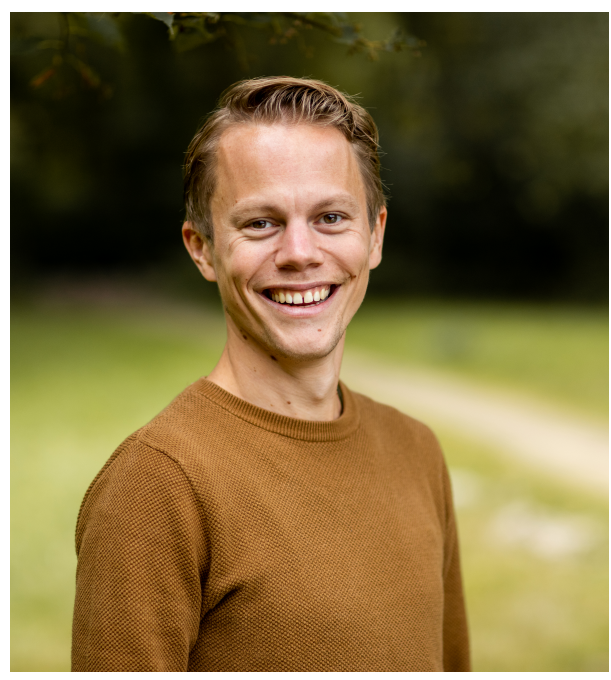
and Antennas group his work comprises modelling and simulation of electromagnetic phenomena in aerospace. This includes propagation of EM waves through radomes and their effects on antenna performance, design of structure integrated antennas and radar absorbing materials. However, his main focus throughout the years at NLR has been aerospace EMC, specifically crosstalk between cables. In the latest years this also includes the EMC challenges in sustainable aviation, such as suppression of EMI introduced by electric propulsion of aircraft.

Since 2017 Jesper has been working on his PhD as a part-time PhD candidate in the Telecommunications group of the University of Twente, later named the Power Electronics and Electromagnetic Compatibility group. 


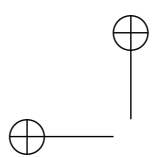

CHAPTER 8. BIOGRAPHY 


\section{References}

[1] European Commission, Flightpath 2050: Europe's vision for aviation. LU: Publications Office, 2011. [Online]. Available: https://data.europa.eu/doi/10.2777/50266

[2] J. König and T. Hellstrom, "The Clean Sky "Smart Fixed Wing Aircraft Integrated Technology Demonstrator": Technology targets and project status," in 27th International Congress of the Aeronautical Sciences, Nice, France, Sep. 2010. [Online]. Available: http://www.icas.org/icas_archive/ICAS2010/papers/288.pdf

[3] Aerospace Technology Institute, "Electrical power systems," Insight, vol. 7, Jul. 2018. [Online]. Available: https://www.ati.org.uk/wpcontent/uploads/2021/09/insight_07-electrical-power-systems.pdf

[4] Aerospace America, "War on wiring," American Institute of Aeronautics and Astronautics, May 2017. [Online]. Available: https://aerospaceamerica.aiaa.org/features/war-on-wiring/

[5] C. R. Paul, Analysis of multiconductor transmission lines. New York: John Wiley \& Sons, 1994.

[6] C. R. Paul and J. Mcknight, "Prediction of Crosstalk Involving Twisted Pairs of Wires-Part I: A Transmission-Line Model for Twisted-Wire Pairs," IEEE Transactions on Electromagnetic Compatibility, vol. EMC-21, no. 2, pp. 92-105, May 1979. [Online]. Available: http://ieeexplore.ieee.org/document/4091262/

[7] C. Jullien, P. Besnier, M. Dunand, and I. Junqua, "Advanced Modeling of Crosstalk Between an Unshielded Twisted Pair Cable and an Unshielded Wire Above a Ground Plane," IEEE Transactions on Electromagnetic Compatibility, vol. 55, no. 1, pp. 183-194, Feb. 2013. [Online]. Available: http://ieeexplore.ieee.org/document/6255786/

[8] J. Clements, C. R. Paul, and A. Adams, "Computation of the Capacitance Matrix for Systems of Dielectric-Coated Cylindrical Conductors," IEEE Transactions on Electromagnetic Compatibility, 
vol. EMC-17, no. 4, pp. 238-248, Nov. 1975. [Online]. Available: http://ieeexplore.ieee.org/document/4090922/

[9] S. Sun, G. Liu, J. L. Drewniak, and D. J. Pommerenke, "HandAssembled Cable Bundle Modeling for Crosstalk and Common-Mode Radiation Prediction," IEEE Transactions on Electromagnetic Compatibility, vol. 49, no. 3, pp. 708-718, Aug. 2007. [Online]. Available: http://ieeexplore.ieee.org/document/4294141/

[10] G. Spadacini, F. Grassi, and S. A. Pignari, "Field-to-Wire Coupling Model for the Common Mode in Random Bundles of Twisted-Wire Pairs," IEEE Transactions on Electromagnetic Compatibility, vol. 57, no. 5, pp. 1246-1254, Oct. 2015. [Online]. Available: http://ieeexplore.ieee.org/document/7076597/

[11] G. Spadacini and S. A. Pignari, "Numerical Assessment of Radiated Susceptibility of Twisted-Wire Pairs With Random Nonuniform Twisting," IEEE Transactions on Electromagnetic Compatibility, vol. 55, no. 5, pp. 956-964, Oct. 2013. [Online]. Available: http://ieeexplore.ieee.org/document/6413194/

[12] P. Manfredi, D. De Zutter, and D. V. Ginste, "Analysis of Nonuniform Transmission Lines With an Iterative and Adaptive Perturbation Technique," IEEE Transactions on Electromagnetic Compatibility, vol. 58, no. 3, pp. 859-867, Jun. 2016. [Online]. Available: http://ieeexplore.ieee.org/document/7405324/

[13] Z. Fei, Y. Huang, J. Zhou, and Q. Xu, "Uncertainty Quantification of Crosstalk Using Stochastic Reduced Order Models," IEEE Transactions on Electromagnetic Compatibility, vol. 59, no. 1, pp. 228-239, Feb. 2017. [Online]. Available: http://ieeexplore.ieee.org/document/7569099/

[14] M. Wu, D. G. Beetner, T. H. Hubing, H. Ke, and S. Sun, "Statistical Prediction of "Reasonable Worst-Case" Crosstalk in Cable Bundles," IEEE Transactions on Electromagnetic Compatibility, vol. 51, no. 3, pp. 842-851, Aug. 2009. [Online]. Available: http://ieeexplore.ieee.org/document/5200329/

[15] F. Diouf and F. Canavero, "Crosstalk statistics via collocation method," in 2009 IEEE International Symposium on Electromagnetic Compatibility (EMC). Austin, TX, USA: IEEE, Aug. 2009, pp. 92-97. [Online]. Available: http://ieeexplore.ieee.org/document/5284686/

[16] D. Bellan and S. A. Pignari, "Efficient Estimation of Crosstalk Statistics in Random Wire Bundles With Lacing Cords," IEEE Transactions on Electromagnetic Compatibility, vol. 53, no. 1, pp. 209-218, Feb. 2011. [Online]. Available: http://ieeexplore.ieee.org/document/5713362/

[17] S. Salio, F. Canavero, D. Lecointe, and W. Tabbara, "Crosstalk prediction on wire bundles by Kriging approach," in 2000 IEEE International 
Symposium on Electromagnetic Compatibility (EMC), vol. 1. Washington, DC, USA: IEEE, Aug. 2000, pp. 197-202. [Online]. Available: http://ieeexplore.ieee.org/document/875562/

[18] L. R. A. X. De Menezes, D. W. P. Thomas, C. Christopoulos, A. Ajayi, and P. Sewell, "The use of Unscented Transforms for statistical analysis in EMC," in 2008 International Symposium on Electromagnetic Compatibility (EMC EUROPE). Hamburg, Germany: IEEE, Sep. 2008, pp. 1-5. [Online]. Available: http://ieeexplore.ieee.org/document/4786803/

[19] C. Jullien, M. Ridel, C. Giraudon, J. Genoulaz, A. Dieudonné, J.-P. Parmantier, S. Bertuol, and C. Girard, "Determination de la diaphonie entre plusieurs harnais complexes au sein d'un avion," in Colloque International et Exposition sur la Compatibilité Électromagnétique (CEM2016), Rennes (France), Jul. 2016, p. 6. [Online]. Available: https://www.researchgate.net/publication/318882370_determination_de_la_ diaphonie_entre_plusieurs_harnais_complexes_au_sein_d'un_avion

[20] Boeing, "Boeing 787 from the ground up," Boeing $A E R O$, vol. Q04, 2006. [Online]. Available: https://www.boeing.com/commercial/aeromagazine/articles/qtr_4_06/ article_04_1.html

[21] Airbus, "Taking the lead: A350XWB," Airbus, p. 28, 2006. [Online]. Available: https://web.archive.org/web/20151211075851/https://www.airbusgroup .com/dam/assets/airbusgroup/int/en/investor-relations/documents/2006/ untitled/further_pre_a350_xwb_launch_2006.pdf

[22] J. Lansink Rotgerink, H. Schippers, and F. Leferink, "Low-Frequency Analysis of Multiconductor Transmission Lines for Crosstalk Design Rules," IEEE Transactions on Electromagnetic Compatibility, vol. 61, no. 5, pp. 1612-1620, Oct. 2019. [Online]. Available: https://ieeexplore.ieee.org/document/8467359/

[23] J. Lansink Rotgerink and H. Schippers, "Crosstalk modelling of unshielded wire pairs," in 2014 International Symposium on Electromagnetic Compatibility (EMC EUROPE). Gothenburg, Sweden: IEEE, Sep. 2014, pp. 641646. [Online]. Available: http://ieeexplore.ieee.org/document/6930984/

[24] F. Happ, F. Gronwald, and H.-D. Bruns, "An extension of Schelkunoff's shielding theory to anisotropic conducting multilayer materials," in 2015 IEEE International Symposium on Electromagnetic Compatibility (EMC EUROPE). Dresden, Germany: IEEE, Aug. 2015, pp. 1542-1547. [Online]. Available: http://ieeexplore.ieee.org/document/7256404/

[25] J. Lansink Rotgerink, F. Happ, and J. J. P. van Es, "Crosstalk between wire pairs above a composite ground plane," in 2016 International Symposium on Electromagnetic Compatibility (EMC EUROPE). 
Wroclaw, Poland: IEEE, Sep. 2016, pp. 89-93. [Online]. Available: http://ieeexplore.ieee.org/document/7739201/

[26] Aerospace Technology Institute, "Composite material applications in aerospace," Insight, vol. 9, Sep. 2018. [Online]. Available: https://www.ati.org.uk/wp-content/uploads/2021/08/insight_9composites_amended-2018-09-20.pdf

[27] C. Paul, "Effect of Pigtails on Crosstalk to Braided-Shield Cables," IEEE Transactions on Electromagnetic Compatibility, vol. EMC-22, no. 3, pp. 161-172, Aug. 1980. [Online]. Available: http://ieeexplore.ieee.org/document/4091369/

[28] J. Lansink Rotgerink, H. Schippers, J. Verpoorte, and K. Nuyten, "EMC aspects of compact wiring for future aircraft," in 2018 IEEE International Symposium on Electromagnetic Compatibility and 2018 IEEE Asia-Pacific Symposium on Electromagnetic Compatibility (EMC/APEMC). Singapore: IEEE, May 2018, pp. 165-170. [Online]. Available: https://ieeexplore.ieee.org/document/8393760/

[29] G. Li, G. Hess, R. Hoeckele, S. Davidson, P. Jalbert, V. V. Khilkevich, T. P. Van Doren, D. Pommerenke, and D. G. Beetner, "MeasurementBased Modeling and Worst-Case Estimation of Crosstalk Inside an Aircraft Cable Connector," IEEE Transactions on Electromagnetic Compatibility, vol. 57, no. 4, pp. 827-835, Aug. 2015. [Online]. Available: http://ieeexplore.ieee.org/document/6985640/

[30] N. Mora, F. Rachidi, P. Pelissou, and A. Junge, "Numerical Simulation of the Overall Transfer Impedance of Shielded Spacecraft Harness Cable Assemblies," IEEE Transactions on Electromagnetic Compatibility, vol. 57, no. 4, pp. 894-902, Aug. 2015. [Online]. Available: http://ieeexplore.ieee.org/document/7054483/

[31] D. White and M. Mardiguian, "Near-field conductor-to-conductor coupling (Crosstalk)," in EMI control methodology and procedures (Handbook series on electromagnetic interference and compatibility, vol. 8). Gainesville: Interference control technologies, 1989.

[32] C. R. Paul, Introduction to electromagnetic compatibility. Hoboken, NJ: Wiley, 2006, oCLC: 836772763. [Online]. Available: http://site.ebrary.com/lib/alltitles/docDetail.action?doclD=10305239

[33] C. R. Paul and J. McKnight, "Prediction of Crosstalk Involving Twisted Pairs of Wires-Part II: A Simplified Low-Frequency Prediction Model," IEEE Transactions on Electromagnetic Compatibility, vol. EMC-21, no. 2, pp. 105-114, May 1979. [Online]. Available: http://ieeexplore.ieee.org/document/4091263/ 
[34] Altair Feko, "(Februari 05, 2020)." [Online]. Available: http://www.altairhyperworks.com/product/feko

[35] J. Lansink Rotgerink and F. Leferink, "A Crosstalk Sensitivity Analysis on Bundles of Twisted Wire Pairs," in 2018 International Symposium on Electromagnetic Compatibility (EMC EUROPE). Amsterdam, the Netherlands: IEEE, Aug. 2018, pp. 11-16. [Online]. Available: https://ieeexplore.ieee.org/document/8485033/

[36] C. Paul and B. Bowles, "Literal solution of the transmission-line equations for shielded wires," in IEEE International Symposium on Electromagnetic Compatibility (EMC). Washington, DC, USA: IEEE, Aug. 1990, pp. 591-599. [Online]. Available: http://ieeexplore.ieee.org/document/252838/

[37] C. Paul, "Transmission-Line MNodeling of Shielded Wires for Crosstalk Prediction," IEEE Transactions on Electromagnetic Compatibility, vol. EMC-23, no. 4, pp. 345-351, Nov. 1981. [Online]. Available: http://ieeexplore.ieee.org/document/4091491/

[38] C. Paul and B. Bowles, "Symbolic solution of the multiconductor transmission-line equations for lines containing shielded wires," IEEE Transactions on Electromagnetic Compatibility, vol. 33, no. 3, pp. 149-162, Aug. 1991. [Online]. Available: http://ieeexplore.ieee.org/document/85128/

[39] H. Schippers, J. Verpoorte, and R. Otin, "Electromagnetic analysis of metal braids," in 2017 International Symposium on Electromagnetic Compatibility (EMC Europe), York, UK, 2011, pp. 543-548. [Online]. Available: https://ieeexplore.ieee.org/document/6078613

[40] J. Lansink Rotgerink, R. Serra, and F. Leferink, "Multiconductor Transmission Line Modeling of Crosstalk Between Cables in the Presence of Composite Ground Planes," IEEE Transactions on Electromagnetic Compatibility, vol. Early Access, pp. 1-9, 2020. [Online]. Available: https://ieeexplore.ieee.org/document/9290410/

[41] J. R. Carson, "Wave Propagation in Overhead Wires with Ground Return," Bell System Technical Journal, vol. 5, no. 4, pp. 539-554, Oct. 1926. [Online]. Available: http://ieeexplore.ieee.org/lpdocs/epic03/wrapper.htm?arnumber=6768096

[42] J. R. Wait, "Theory of Wave Propagation Along a Thin Wire Parallel to An Interface," Radio Science, vol. 7, no. 6, pp. 675-679, Jun. 1972. [Online]. Available: http://doi.wiley.com/10.1029/RS007i006p00675

[43] E. Sunde, Earth conduction effects in transmission lines. New York: Dover, 1968.

[44] L. M. Wedepohl, "Wave propagation in multiconductor overhead lines," vol. 113, no. 4, p. 6, 1966. 
[45] F. Rachidi, S. Loyka, C. Nucci, and M. lanoz, "A new expression for the ground transient resistance matrix elements of multiconductor overhead transmission lines," Electric Power Systems Research, vol. 65, no. 1, pp. 41-46, Apr. 2003. [Online]. Available: https://linkinghub.elsevier.com/retrieve/pii/S0378779602002146

[46] M. D'Amore and M. S. Sarto, "A new formulation of lossy ground return parameters for transient analysis of multiconductor dissipative lines," IEEE Transactions on Power Delivery, vol. 12, no. 1, pp. 303-314, Jan. 1997. [Online]. Available: https://ieeexplore.ieee.org/document/568254

[47] F. Rachidi, C. Nucci, and M. Ianoz, "Transient analysis of multiconductor lines above a lossy ground," IEEE Transactions on Power Delivery, vol. 14, no. 1, pp. 294-302, Jan. 1999. [Online]. Available: http://ieeexplore.ieee.org/document/736741/

[48] C. R. Paul, "Solution of the transmission-line equations for lossy conductors and imperfect earth," Proceedings of the Institution of Electrical Engineers, vol. 122, no. 2, p. 177, 1975. [Online]. Available: https://digital-library.theiet.org/content/journals/10.1049/piee.1975.0040

[49] F. Rachidi, "A Review of Field-to-Transmission Line Coupling Models With Special Emphasis to Lightning-Induced Voltages on Overhead Lines," IEEE Transactions on Electromagnetic Compatibility, vol. 54, no. 4, pp. 898-911, Aug. 2012. [Online]. Available: http://ieeexplore.ieee.org/document/6146418/

[50] C. R. Paul, "A Brief History of Work in Transmission Lines for EMC Applications," IEEE Transactions on Electromagnetic Compatibility, vol. 49, no. 2, pp. 237-252, May 2007. [Online]. Available: http://ieeexplore.ieee.org/document/4244615/

[51] T. Demeester and D. De Zutter, "Quasi-TM Transmission Line Parameters of Coupled Lossy Lines Based on the Dirichlet to Neumann Boundary Operator," IEEE Transactions on Microwave Theory and Techniques, vol. 56, no. 7, pp. 1649-1660, Jul. 2008. [Online]. Available: http://ieeexplore.ieee.org/document/4538249/

[52] M. Kechicheb, M. R. Mekideche, and C. Edith, "Electromagnetic Modeling of Wire-Ground Structures by using a MTL Based Approach," Automatika, vol. 57, no. 4, pp. 1035-1044, Oct. 2016. [Online]. Available: https://www.tandfonline.com/doi/full/10.7305/automatika.2017.12.1542

[53] N. Mora, G. Lugrin, F. Rachidi, I. Junqua, J. Parmantier, S. Tkachenko, M. Rubinstein, M. Nyffeler, and P. Bertholet, "On the validity limits of the transmission line theory in evaluating differential-mode signals along a two-wire line above a ground plane," in 2015 IEEE International Symposium on Electromagnetic Compatibility (EMC). 
Dresden, Germany: IEEE, Aug. 2015, pp. 797-800. [Online]. Available: http://ieeexplore.ieee.org/document/7256265/

[54] C. Holloway, M. Sarto, and M. Johansson, "Analyzing Carbon-Fiber Composite Materials With Equivalent-Layer Models," IEEE Transactions on Electromagnetic Compatibility, vol. 47, no. 4, pp. 833-844, Nov. 2005. [Online]. Available: http://ieeexplore.ieee.org/document/1580753/

[55] C. L. Holloway, E. F. Kuester, A. E. Ruehli, and G. Antonini, "Partial and Internal Inductance: Two of Clayton R. Paul's Many Passions," IEEE Transactions on Electromagnetic Compatibility, vol. 55, no. 4, pp. 600-613, Aug. 2013. [Online]. Available: http://ieeexplore.ieee.org/document/6507331/

[56] C. R. Paul, "Sensitivity of Crosstalk to Variations in Wire Position in Cable Bundles," in 1987 IEEE International Symposium on Electromagnetic Compatibility. Atlanta, Georgia, USA: IEEE, Aug. 1987, pp. 1-5. [Online]. Available: http://ieeexplore.ieee.org/document/7570814/

[57] Z. Fei, Y. Huang, J. Zhou, and Q. Xu, "Sensitivity analysis of cable crosstalk to uncertain parameters using stochastic reduced order models," in 2016 IEEE International Symposium on Electromagnetic Compatibility (EMC), Ottawa, Canada, Jul. 2016.

[58] Z. Fei, Y. Huang, J. Zhou, and C. Song, "Numerical Analysis of a Transmission Line Illuminated by a Random Plane-Wave Field Using Stochastic Reduced Order Models," IEEE Access, vol. 5, pp. 8741-8751, 2017. [Online]. Available: http://ieeexplore.ieee.org/document/7921534/

[59] J. Lansink Rotgerink, H. Schippers, and J. Verpoorte, "Multi-conductor transmission line modelling of transfer impedance measurement methods," in 2017 International Symposium on Electromagnetic Compatibility (EMC EUROPE). Angers, France: IEEE, Sep. 2017, pp. 1-7. [Online]. Available: http://ieeexplore.ieee.org/document/8094778/

[60] J. Lansink Rotgerink, J. Verpoorte, and H. Schippers, "Uncertainties in coaxial cable transfer impedance," IEEE Electromagnetic Compatibility Magazine, vol. 7, no. 3, pp. 83-93, 2018. [Online]. Available: https://ieeexplore.ieee.org/document/8479344/

[61] H. Schippers and J. Verpoorte, "Uncertainties in transfer impedance calculations," in 2016 ESA Workshop on Aerospace EMC (Aerospace EMC). Valencia, Spain: IEEE, May 2016, pp. 1-6. [Online]. Available: http://ieeexplore.ieee.org/document/7504589/

[62] S. Schelkunoff, "The electromagnetic theory on coaxial transmission lines and cylindrical shields," Bell Systems Technical Journal, vol. 14, no. 3, pp. 532-579, Oct. 1934. 
[63] E. Vance, "Shielding Effectiveness of Braided-Wire Shields," IEEE Transactions on Electromagnetic Compatibility, vol. EMC-17, no. 2, pp. 71-77, May 1975. [Online]. Available: http://ieeexplore.ieee.org/document/4090877/

[64] T. Kley, "Optimized single-braided cable shields," IEEE Transactions on Electromagnetic Compatibility, vol. 35, no. 1, pp. 1-9, Feb. 1993. [Online]. Available: http://ieeexplore.ieee.org/document/249390/

[65] M. Tyni, "The Transfer Impedance of Coaxial Cables with Braided Outer Conductor," FV. Nauk, Inst. Telekomum Akust. Politech Wroclaw, Ser. Konfi,, pp. 410-419, 1975.

[66] EN 50289-1-6: 2002 E, "Communication cables - Specifications for test methods Part 1-6: Electrical test methods - Electromagnetic performance," CENELEC, Mar. 2002.

[67] IEC 62153-4-3, "Metallic communication cable test methods - Part 4-3: Electromagnetic compatibility (EMC) - Surface transfer impedance - Triaxial method," IEC, Mar. 2006.

[68] IEC 62153-4-6, "Metallic communication cable test methods - Part 4-6: Electromagnetic compatibility (EMC) - Surface transfer impedance - Line injection method," IEC, May 2006.

[69] T. Hähner and B. Mund, "Background, content and future of the EMC measurement standard prEN 50289-7-6, Open / shielded test methods," in International Wroclaw Symposium on Electromagnetic Compatibility, Wroclaw, Poland, 2000.

[70] B. Démoulin and L. Koné, "Shielded cable transfer impedance measurements," IEEE EMC newsletter, pp. 30-37, 2010.

[71] _ - "Shielded Cable Transfer Impedance Measurements in the Microwave Range of $1 \mathrm{GHz}$ to $10 \mathrm{GHz}$," IEEE EMC newsletter, pp. 52-61, 2011.

[72] __, "Shielded Cable Transfer Impedance Measurements High frequency range 100 MHz-1 GHz," IEEE EMC newsletter, pp. 42-50, 2011.

[73] S. Sali, "A MATCHED TRIAXIAL DEVICE FOR CABLE SHIELDING MEASUREMENTS," Progress In Electromagnetics Research, vol. 45, pp. 21-44, 2004. [Online]. Available: http://www.jpier.org/PIER/pier.php?paper=0303261

[74] J. Lansink Rotgerink and $H$. Schippers, "Generic prediction of crosstalk between shielded wires," in 2015 IEEE International Symposium on Electromagnetic Compatibility (EMC EUROPE). Dresden, Germany: IEEE, Aug. 2015, pp. 616-621. [Online]. Available: http://ieeexplore.ieee.org/document/7256234/ 


\section{List of my publications}

\section{Peer-reviewed journals and magazines}

1. J. Lansink Rotgerink, H. Schippers, and F. Leferink, "Low-Frequency Analysis of Multiconductor Transmission Lines for Crosstalk Design Rules," IEEE Transactions on Electromagnetic Compatibility, vol. 61, no. 5, pp. 1612-1620, Oct. 2019. [Online]. Available: https://ieeexplore.ieee.org/ document/8467359/

2. J. Lansink Rotgerink, R. Serra, and F. Leferink, "Multiconductor Transmission Line Modeling of Crosstalk Between Cables in the Presence of Composite Ground Planes," IEEE Transactions on Electromagnetic Compatibility, Vol. 63, no. 4, pp. 1231-1239, Aug. 2021. [Online]. Available: https:// ieeexplore.ieee. org/document/9290410/

3. J. Lansink Rotgerink, J. Verpoorte, and H. Schippers, "Uncertainties in coaxial cable transfer impedance," IEEE Electromagnetic Compatibility Magazine, vol. 7, no. 3, pp. 83-93, 2018. [Online]. Available: https://ieeexplore. ieee.org/document/8479344/

\section{Peer-reviewed conferences}

1. J. Lansink Rotgerink and H. Schippers, "Crosstalk modelling of unshielded wire pairs," in 2014 International Symposium on Electromagnetic Compatibility (EMC EUROPE). Gothenburg, Sweden: IEEE, Sep. 2014, pp. 641646. [Online]. Available: http://ieeexplore.ieee.org/document/6930984/

2. - " "Generic prediction of crosstalk between shielded wires," in 2015 IEEE International Symposium on Electromagnetic Compatibility (EMC EUROPE). Dresden, Germany: IEEE, Aug. 2015, pp. 616-621. [Online]. Available: http://ieeexplore.ieee.org/document/7256234/

3. J. Lansink Rotgerink and J. Verpoorte, "Low-frequency closed-form expressions for crosstalk between twisted wire pairs," in 2016 ESA Workshop 
on Aerospace EMC (Aerospace EMC). Valencia, Spain: IEEE, May 2016, pp. 1-6. [Online]. Available: http://ieeexplore.ieee.org/document/7504555/

4. J. Lansink Rotgerink, F. Happ, and J.J.P. van Es, "Crosstalk between wire pairs above a composite ground plane," in 2016 International Symposium on Electromagnetic Compatibility (EMC EUROPE). Wroclaw, Poland: IEEE, Sep. 2016, pp. 89-93. [Online]. Available: http://ieeexplore.ieee.org/ document/7739201/

5. J. Lansink Rotgerink, H. Schippers, and J. Verpoorte, "Multi-conductor transmission line modelling of transfer impedance measurement methods," in 2017 International Symposium on Electromagnetic Compatibility (EMC EUROPE). Angers, France: IEEE, Sep. 2017, pp. 1-7. [Online]. Available: http://ieeexplore.ieee.org/document/8094778/

6. J. Lansink Rotgerink, H. Schippers, J. Verpoorte, and K. Nuyten, "EMC aspects of compact wiring for future aircraft," in 2018 IEEE International Symposium on Electromagnetic Compatibility and 2018 IEEE Asia-Pacific Symposium on Electromagnetic Compatibility (EMC/APEMC). Singapore: IEEE, May 2018, pp. 165-170. [Online]. Available: https://ieeexplore.ieee.org/ document/8393760/

7. N. Moonen, J. Lansink Rotgerink, and F. Leferink, "Automated equivalent circuit extraction of impedance curves using a Gauss-Newton algorithm," in 2018 IEEE International Symposium on Electromagnetic Compatibility and 2018 IEEE Asia-Pacific Symposium on Electromagnetic Compatibility (EMC/APEMC). Singapore: IEEE, May 2018, pp. 1103-1108. [Online]. Available: https://ieeexplore.ieee.org/document/8393958/

8. J. Verpoorte, H. Schippers, and J. Lansink Rotgerink, "Advanced models for the transfer impedance of metal braids in cable harnesses," in 2018 IEEE International Symposium on Electromagnetic Compatibility and 2018 IEEE Asia-Pacific Symposium on Electromagnetic Compatibility (EMC/APEMC). Singapore: IEEE, May 2018, pp. 187-192. [Online]. Available: https://ieeexplore.ieee.org/document/8393764/

9. J. Lansink Rotgerink and F. Leferink, "A Crosstalk Sensitivity Analysis on Bundles of Twisted Wire Pairs," in 2018 International Symposium on Electromagnetic Compatibility (EMC EUROPE). Amsterdam, the Netherlands: IEEE, Aug. 2018, pp. 11-16. [Online]. Available: https://ieeexplore.ieee.org/ document/8485033/

10. J. Lansink Rotgerink, N. Moonen, and F. Leferink, "Mixed-Mode S-Parameter Measurements for Determination of Cable Coupling," in 2019 ESA Workshop on Aerospace EMC (Aerospace EMC). Budapest, Hungary: IEEE, May 2019, pp. 1-6. [Online]. Available: https://ieeexplore.ieee.org/document/ 8788948/ 
LIST OF MY PUBLICATIONS

11. J. Lansink Rotgerink, G. Erotas, N. Moonen, and F. Leferink, "Comparing Various Measurement and Simulation Techniques for Estimating Crosstalk," in 2020 International Symposium on Electromagnetic Compatibility (EMC EUROPE). Rome, Italy: IEEE, Sep. 2020, pp. 1-6. [Online]. Available: https://ieeexplore.ieee.org/document/9245852/ 


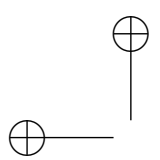

LIST OF MY PUBLICATIONS

(1) $\emptyset$ 